\title{
Tamoxifen and beyond : antiestrogens and aromatase inhibitors in the hormonal therapy of breast cancer
}

Citation for published version (APA):

Bertelli, G. (2007). Tamoxifen and beyond : antiestrogens and aromatase inhibitors in the hormonal therapy of breast cancer. [Doctoral Thesis, Maastricht University]. Datawyse / Universitaire Pers Maastricht. https://doi.org/10.26481/dis.20070215gb

Document status and date:

Published: 01/01/2007

DOI:

10.26481/dis.20070215gb

Document Version:

Publisher's PDF, also known as Version of record

\section{Please check the document version of this publication:}

- A submitted manuscript is the version of the article upon submission and before peer-review. There can be important differences between the submitted version and the official published version of record.

People interested in the research are advised to contact the author for the final version of the publication, or visit the DOI to the publisher's website.

- The final author version and the galley proof are versions of the publication after peer review.

- The final published version features the final layout of the paper including the volume, issue and page numbers.

Link to publication

\footnotetext{
General rights rights.

- You may freely distribute the URL identifying the publication in the public portal. please follow below link for the End User Agreement:

www.umlib.nl/taverne-license

Take down policy

If you believe that this document breaches copyright please contact us at:

repository@maastrichtuniversity.nl

providing details and we will investigate your claim.
}

Copyright and moral rights for the publications made accessible in the public portal are retained by the authors and/or other copyright owners and it is a condition of accessing publications that users recognise and abide by the legal requirements associated with these

- Users may download and print one copy of any publication from the public portal for the purpose of private study or research.

- You may not further distribute the material or use it for any profit-making activity or commercial gain

If the publication is distributed under the terms of Article $25 \mathrm{fa}$ of the Dutch Copyright Act, indicated by the "Taverne" license above, 
TAMOXIFEN AND BEYOND:

ANTIESTROGENS AND AROMATASE INHIBITORS

IN THE HORMONAL THERAPY OF BREAST CANCER 
Copyright ( Gianfilippo Bertelli, Swansea UK, 2006

ISBN-10: 90-5278-546-5

ISBN-13: 978-90-5278-546-2

Production: Datawyse / Universitaire Pers Maastricht

Cover: Flora, Titian (Galleria degli Uffizi, Florence)

This Thesis was supported in part by educational grants from Novartis UK and Pfizer UK. 
TAMOXIFEN AND BEYOND:

\title{
ANTIESTROGENS AND AROMATASE INHIBITORS IN
} THE HORMONAL THERAPY OF BREAST CANCER

\author{
Proefschrift \\ ter verkrijging van de graad van doctor \\ aan de Universiteit Maastricht \\ op gezag van de Rector Magnificus, \\ Prof. mr. G.P.M.F. Mols \\ volgens het besluit van het College van Decanen, \\ in het openbaar te verdedigen \\ op donderdag 15 februari 2007 om 16.00 uur \\ door \\ Gianfilippo Bertelli
}

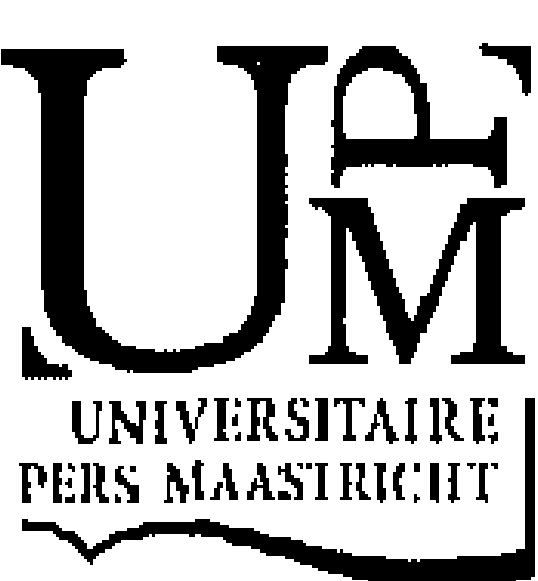


Promotor:

Prof. dr. J. Wagstaff

\section{Promoticcommissie:}

Prof. dr. H.F.P. Hillen, voorzitter Prof. dr. J. De Haan Dr. P.S.G.J. Hupperets Prof. dr. J.G.M. Klijn Prof. dr. R.C.F. Leonard Prof. dr. M.F. von Meyenfeldt 


\section{Contents}

\section{Chapter 1}

Introduction and scope of the Thesis

\section{Chapter 2}

Tamoxifen and the endometrium: findings of pelvic ultrasound examination and endometrial biopsy in asymptomatic breast cancer patients.

Breast Cancer Res. Treat. 1998;47:41-6.

\section{Chapter 3}

Limited value of sonohysterography for endometrial screening in asymptomatic, postmenopausal patients treated with tamoxifen.

Gynecol. Oncol. 20(0);7:275-7.

\section{Chapter 4}

Toremifene as a substitute for adjuvant tamoxifen in breast cancer patients. Anticancer Res. 2000;20:3659-61.

\section{Chapter 5}

Intramuscular depot medroxyprogesterone versus oral megestrol for the control of postmenopausal hot flashes in breast cancer patients: a randomized study. Ann. Oncol. 2002;13:883-8.

\section{Chapter 6}

A randomized trial of exemestane after two to three years of tamoxifen therapy in postmenopausal women with primary breast cancer.

N. Engl. J. Med. 2004:350:1081-92.

\section{Chapter 7}

IES trial: endometrial sub study.

San Antonio Brecist Cancer. Symposium, December 2004

\section{Chapter 8}

Maintenance hormone therapy with letrozole after first-line chemotherapy for advanced breast cancer.

Oncology 2005;68:364-370.

\section{Chapter 9}

Sequential treatment with exemestane and non-steroidal aromatase inhibitors in advanced breast cancer.

Oncology 2005; 69:471-477. 
Chapter 10

Conclusions: New paradigms in hormonal therapy of breast cancer

Summary

101

Curriculum Vitae \& Publications 


\section{Chapter 1}

Introduction and scope of the Thesis 
Since the pioneering description by George Beatson of the beneficial effects of estrogen deprivation in breast cancer [ 1 ], hormonal therapy of early and advanced breast cancer has developed into a complex therapeutic field where multiple agents are available and treatment strategies are continuously being refined. Historically, the major phases of this development have been the abandonment of ablative endocrine treatments (hypophysectomy and adrenalectomy) in favour of additive pharmacological therapy, mainly aimed at the suppression of estrogen synthesis or action; the identification of the estrogen receptor expression in tumour cells as a crucial predictive factor for response; and the establishment of the antiestrogen tamoxifen as the gold standard of hormonal therapy. More recently, the role of tamoxifen has been challenged by selective aromatase inhibitors, which may be seen as the protagonists of a new phase of the evolution of hormonal therapy of breast cancer which is currently still in progress.

The scope of this Thesis is to present the results of studies on the hormonal therapy of breast cancer, performed at the Medical Oncology Department of the National Cancer Institute in Genova and at the Medical Oncology Unit of the S.Croce Hospital in Cuneo, Italy, mainly within the framework of the cooperative group GONO (Gruppo Oncologico Nord Ovest). Some of the studies have been continued and completed at the South West Wales Cancer Institute in Swansea, UK, which is my current place of work. The studies have focused on two main topics: tamoxifen and its estrogenic and antiestrogenic side effects, and the role of third-generation aromatase inhibitors in improving outcomes and ameliorating toxicity in early and advanced breast cancer.

Estrogenic side effects of tamoxifen. Although tamoxifen was introduced into clinical use as an antiestrogen [2], it is now better described as a selective estrogen receptor modulator (SERM) with mixed antagonist/agonist actions that differ from tissue to tissue. Tamoxifen's main therapeutic action is an antiestrogenic effect on breast cancer resulting in reduced risk of recurrence and mortality when used as adjuvant treatment in early disease [3], and for palliative benefit in advanced disease. Its partial estrogen-like activity is however associated with potentially severe adverse effects, such as increased risk of venous thrombosis and endometrial cancer. The risk of thrombosis is especially increased when tamoxifen is used in combination with chemotherapy [4] but has also been observed in chemoprevention trials where tamoxifen was used in healthy women [5]. The mechanisms of the pro-thrombotic effect of tamoxifen are unclear, but a decrease in the levels of anticoagulant proteins such as antithrombin-III and protein-C have been described by our group and others [6-8].

Numerous studies since the mid-80s have shown an association between tamoxifen and endometrial cancer. In a metaanalysis of 32 randomised trials where tamoxifen was included in one of the treatment arms, the relative risk ratio for endometrial cancer with tamoxifen was 2.7 (95\% C.I., 1.94 to 3.75) [9]. The risk of endometrial cancer increases with longer duration of tamoxifen therapy, and persists for some time after tamoxifen withdrawal. In a recent British case-control study, 816 patients who had endometrial cancer after a diagnosis of breast cancer were compared to 1067 patients who had breast cancer but no endometrial cancer [10]. The risk associated with tamoxifen significantly increased with duration of exposure (ORs for 2 to 4,5 to 7,8 to 9 , and 10 to 17 years of therapy were 2.1,2.5, 4.8 and 7.9, respectively), and did not diminish for up to at least 5 years after the end of treatment. 
In the first reports of tamoxifen-associated endometrial neoplasia, most patients had early stage, well- or moderately differentiated endometrial adenocarcinomas. With longterm follow-up of patients exposed to tamoxifen, more recent studies have however also highlighted an increased incidence of poor-risk carcinomas and of sarcomas. Data from the Surveillance, Epidemiology, and End Results (SEER) database of the National Cancer Institute on 39,54I breast cancer patients treated with tamoxifen show an eightfold increase in the relative risk of malignant mixed mullerian tumors (MMMTs) in patients surviving five years or longer [11]. Because of their rarity, the absolute number of tamoxifen-associated MMMTs is low, but their prognosis is worse than that of adenocarcinomas.

Tamoxifen is also associated with a number of non-malignant uterine changes, including endometrial hyperplasia, adenomyosis, endometrial polyps, glandulocystic atrophy, and stromal condensation [12]. Of these, only atypical endometrial hyperplasia has an established premalignant significance, while the remainder have an uncertain relationship with the development of malignancy. Glandulocystic atrophy, characterized by atrophic endometrium and multiple cysts in a dense, fibrous stroma is the most common abnormality of the endometrium in postmenopausal patients receiving tamoxifen. The incidence of endometrial polyps varies between 12 and $25 \%$. Tamoxifen-associated polyps are also often characterized by atrophic epithelium and glandulocystic dilation with periglandular stromal condensation.

Endometrial screening in patients on tamoxifen. In women with breast cancer, the benefits of tamoxifen outweigh the increased risk of endometrial toxicity. However, patients and their clinicians are often understandably anxious about the risk of a second, drug-induced malignancy, and many efforts have been made to explore screening strategies in this population. Ultrasound scans are useful in assessing the endometrium in normal postmenopausal women, where an endometrial thickness of more than 3-4 millimetres is considered abnormal. Several groups have investigated the value of pelvic or transvaginal ultrasound screening in patients on tamoxifen [13-16]. For example, in a study by Gerber et al, the mean endometrial thickness increased from 3.5 to $9.2 \mathrm{~mm}$ during treatment with adjuvant tamoxifen in 247 postmenopausal patients who were prospectively assessed with transvaginal ultrasound [17]. Fifty-two asymptomatic patients with thickened or morphologically suspect endometrium underwent hysteroscopy with dilatation and curettage (D\&C), resulting in four uterine perforations. One case of endometrial cancer and one of atypical hyperplasia were found, while the remaining patients had atrophic endometrium (73\%), benign polyps or simple hyperplasia. Studies have also investigated the use of office endometrial biopsy as a screening tool in patients on tamoxifen. Biopsies are however more invasive than ultrasound, and the yield of significant endometrial pathology remains low in asymptomatic subjects [18].

The study presented in chapter 2 was designed to shed further light on the incidence of endometrial pathology in asymptomatic women with breast cancer either on no hormone therapy or taking tamoxifen and to further elucidate the role of pelvic ultrasound and endometrial biopsy in screening these women for endometrial pathology [19]. In chapter 3 the use of an alternative technique, namely sonohysterography, to improve the sensitivity of ultrasound to detect endometrial pathology was examined [20]. 
Reduction of uterine toxicity of tamoxifen: studies on toremifene and progestins. The potential strategies for the reduction of uterine toxicity of hormonal therapy in breast cancer include the substitution of tamoxifen with other agents devoid of this effect, and the combined use of tamoxifen with progestins to protect the endometrium from its stimulating effect. With regard to the first strategy, before the availability of third-generation aromatase inhibitors as possible alternatives to tamoxifen, attempts were made to substitute tamoxifen with other SERMs. Although raloxifene, a SERM with an interesting profile of estrogenic activity on bones and lipids and lack of estrogenic action on the uterus [21], is being studied in the chemopreventive setting, it is not currently licensed for breast cancer patients. Toremifene was introduced into the clinic as having similar clinical efficacy to tamoxifen, but a lower in vitro potential for carcinogenicity $[22,23]$. The study presented in chapter 4 was designed to examine the effects on the uterus of a cross-over from tamoxifen to toremifene in patients receiving adjuvant hormonal therapy for early breast cancer [24], by prospectively assessing the uterus with transvaginal ultrasound. The other potential strategy, i.e. the addition of a progestin to tamoxifen in an attempt to protect the endometrium from its estrogen-like effects, has been advocated by some investigators. Cyclic oral norethisterone however was not effective in reversing tamoxifen-associated ultrasound abnormalities in healthy postmenopausal women participating to a chemopreventive trial [25]. In our experience on postmenopausal patients on adjuvant tamoxifen for early breast cancer, oral megestrol acetate also failed to reverse or prevent tamoxifen-induced abnormalities [26]. On the other hand, a levonorgestrel-releasing intrauterine system seemed to have protective effects in a study on postmenopausal patients on adjuvant tamoxifen [27]. This combination could therefore be worth exploring further.

Anti-estrogenic side effects of tamoxifen: menopausal symptoms. While the effects on plasma lipids, coagulation and endometrium are associated with the partial agonist properties of tamoxifen, vasomotor symptoms are exacerbated by tamoxifen in postmenopausal women as a consequence of its anti-estrogen activity. Approximately $50 \%$ of patients report hot flushes and night sweats during treatment with tamoxifen [28], which may impact significantly on the quality of life of patients and on their compliance to treatment. Estrogens can be very effective in the relief of hot flushes in postmenopausal women, but their use is not generally recommended for patients with a history of breast cancer. Progestational agents can also ameliorate hot flushes. In a randomized, placebo controlled trial, low-dose oral megestrol acetate significantly reduced the frequency and severity of hot flashes in women with breast cancer and men with prostate cancer [29]. In chapter 5 we have compared low-dose oral megestrol acetate with intramuscular medroxyprogesterone acetate in a randomized trial in postmenopausal patients with early breast cancer suffering from hot flushes [30]. The aim was to determine whether it was possible to further ameliorate this troublesome side effects of tamoxifen making it more tolerable for women.

Beyond tamoxifen: third-generation aromatase inhibitors. Since the recognition that peripheral conversion of androgenic precursors by the aromatase enzyme is the main source of estrogens in postmenopausal women, aromatase inhibition - initially with aminoglutethimide, and subsequently with more selective and powerful agents -- has become one of the most interesting pharmacological strategies to develop in breast 
cancer [31]. Between 1996 and 2001, a series of controlled trials have explored the role of the third-generation aromatase inhibitors (AIs) anastrozole, letrozole and exemestane after failure of tamoxifen in advanced breast cancer [32-36]. As compared to megestrol acetate (MA), considered previously the standard of care in this setting, Als proved equivalent or superior to MA in several tolerability and efficacy endpoints. The following phase of the clinical development of AIs was as first-line treatment in advanced disease, where anastrozole, letrozole and exemestane again produced equivalent or superior results to the standard comparator, which in this case was tamoxifen [37-40].

Based on the encouraging results obtained in the treatment of advanced disease, several large trials, some of which are still ongoing, have evaluated the role of adjuvant AIs in postmenopausal women with early breast cancer. The Arimidex, Tamoxifen Alone or in Combination (ATAC) trial [41] compared 5 years of tamoxifen versus five years of anastrozole. With a median follow up of 68 months, the trial shows that adjuvant anastrozole improved event-free survival by $3.7 \%$ compared to tamoxifen, while the combination of tamoxifen and anastrozole has no additional benefit over tamoxifen alone. The Breast International Group (BIG) 1-98 trial [42] also investigated the upfront use of an $\mathrm{AI}$, in this case letrozole, and found a $3.4 \%$ improvement in event-free survival after a median follow up of 25 months.

Other trials have explored a sequential approach, using an AI after initial tamoxifen treatment [43-45]. There are several theoretical reasons to suggest a benefit of sequential endocrine therapy involving switching from tamoxifen to an AI after two to three years. First, many patients with breast cancer have a relapse and die of metastatic disease within five years after the initial diagnosis. Second, in both patients with primary cancer and those with metastatic disease, resistance occurs as early as 12 to 18 months after the initiation of tamoxifen therapy. In some patients with resistant disease, tamoxifen may act as an agonist, potentially stimulating the division of breast-cancer cells. Third, serious side effects of tamoxifen, including thromboembolism and uterine carcinoma, can occur after prolonged use. Fourth, since tamoxifen can decrease bone resorption [46], we reasoned that pretreatment with tamoxifen might lessen the effect of any osteopenia caused by exemestane. For these reasons the Intergroup Exemestane Study (IES) [47] was designed to examine the efficacy of randomly switching to exemestane after two to three years of tamoxifen. The results of this study are presented in chapter 6 .

Aromatase inhibitors and the endometrium. Since AIs are completely devoid of estrogenic activity, avoidance of tamoxifen-associated endometrial problems is one of the potential benefits when AIs are used instead of tamoxifen. In the ATAC trial, patients treated with anastrozole had a significant lower incidence of endometrial cancer $(0.2 \%$ vs. $0.8 \%, p=0.02)$, vaginal bleeding $(5.4 \%$ vs. $10.2 \%, p<0.0001)$ and vaginal discharge $(3.5 \%$ vs. $13.2 \%, p<0.0001)$ compared to patients on tamoxifen [41]. In the BIG 1-98 trial, patients on letrozole had significantly less vaginal bleeding $(3.3 \% \mathrm{vs}$. $6.6 \%, \mathrm{p}<0.001)$ and endometrial biopsies $(2.3 \%$ vs. $9.1 \%, \mathrm{p}<0.001)$, and a nonsignificant reduction of endometrial cancers $0.1 \%$ vs. $0.3 \%, p=0.18$ ) compared to patients on tamoxifen [42].

The analysis of trials exploring the sequential use of Als after tamoxifen is more complex. With a sequential treatment, all patients are exposed to tamoxifen initially, and may therefore develop tamoxifen-induced endometrial abnormalities before switching 
to an Al. Although further follow up is needed, early results of the sequential trials suggest that the endometrial effects of tamoxifen are at least in part reversible. In the MA. 17 trial, patients on letrozole had significantly less vaginal bleeding ( $6 \%$ vs. $8 \%$, $\mathrm{p}=0.005$ ) and a non-significant reduction of endometrial cancers ( 4 cases vs. 11 , $\mathrm{p}=0.12$ ) compared to patients in the placebo arm [45]. In the ABCSG/ARNO trial, there was no difference in vaginal bleeding/discharge rates $(18 \%$ vs. $17 \%, p=0.9)$ and a nonsignificant difference in endometrial cancers $(1$ case vs $7, p=0.06)$ in patients switching to anastrozole compared to those continuing tamoxifen [43].

In the IES trial, a significant reduction of gynaecologic symptoms $(5.8 \%$ vs. $9.0 \%$, $\mathrm{p}<0.001)$ and a non-significant reduction of vaginal bleeding $(4.0 \%$ vs. $5.5 \%, p=0.05)$ and endometrial cancers ( 5 cases vs. 11 ) were observed in patients changed to exemestane compared to those who continued with tamoxifen [47].

As mentioned above, many of the endometrial abnormalities associated with tamoxifen may remain asymptomatic and be missed. Due to its design, the IES trial provided an ideal framework to investigate in more depth the effects on the uterus of changing from tamoxifen to an AI. An endometrial substudy was set up in the IES trial, in which 219 patients were assessed with a baseline transvaginal ultrasound scan at study entry. The ultrasound scan was repeated at 6,12 and 24 months following randomization, at study end, and at 12 and 24 months after study end. The primary endpoint of the study was to be the comparison of the proportion of patients with thickened endometrium ( $\geq 5 \mathrm{~mm}$ ) in the two groups at 24 months from randomization. Other endpoints were to include a comparison of mean uterine volume and mean endometrial thickness in the two groups. The preliminary results of this study were presented at the 2004 San Antonio Breast Cancer Symposium and are the subject matter of chapter 7 .

Sequential use of hormonal agents. The availability of several, well-tolerated hormonal agents has changed many of the traditional paradigms in the treatment of breast cancer. Until recently, tamoxifen was the standard treatment for advanced and early breast cancer. In case of de novo or acquired resistance to tamoxifen, patients could be offered second-line treatment with an AI, and in some cases third-line therapy with a progestin. Today, this sequence has become more complex as a consequence of the growing role of AIs in the adjuvant therapy of early breast cancer and as first-line treatments for advanced disease. Many patients with ER+ disease receive chemotherapy at some point during their treatment for advanced disease. In these patients, the use of hormonal therapy in sequence with chemotherapy is a possible strategy to maintain the benefits of chemotherapy for a longer time. This is an attractive alternative to watchful waiting without treatment until progression, but until recently only one prospective study (with medroxyprogesterone acetate) was available to support it [48]. In order to shed further light on the utility of using maintenance hormone therapy after induction of a remission with chemotherapy we conducted a study of maintenance letrozole in this situation. The data generated from this study have been published [49] and are presented in chapter 8.

In another study, we have investigated the sequential use of exemestane and nonsteroidal Als in advanced breast cancer (chapter 9). It is well known that anti-aromatase agents can be divided into two categories, steroidal inactivators and non-steroidal inhibitors, based on their chemical structure and mechanism of action. Partial non-cross resistance between steroidal and non-steroidal AIs was previously reported in studies of 
exemestane used after non-steroidal Ais [50,51]. Our study was however the first to also examine the opposite sequence (exemestane first, then letrozole or anastrozole) [52].

The clinical implications of the sequencing studies are that anti-aromatase drugs of the two types could be used at different times to prolong disease control, before changing to other, less tolerable treatments (such as progestins or chemotherapy). The choice of first- and subsequent-line hormone therapy for advanced disease should today be based on the treatment history of the patient, on the data relating to non-cross resistance of the various agents, and on a balance of the expected benefits and toxicities with the agent of choice. The impact of new agents, such as fulvestrant [53], on drug sequencing will also need to be clarified. Data presented in this thesis have demonstrated how both the tolerance and effectiveness of the hormone therapy of breast cancer patients can be optimized by conducting careful clinical studies.

\section{References}

1. Beatson GT: On the treatment of inoperable cases of carcinoma of the mamma: Suggestions for a new method of treatment, with illustrative cases. Lancet 1896; 2:102-104.

2 Ward HW. Anti-oestrogen therapy for breast cancer: a trial of tamoxifen at two dose levels. Br Med J 1973; 1:13-4.

3. Early Breast Cancer Trialists' Collaborative Group (EBCTCG). Effects of chemotherapy and hormonal therapy for early breast cancer on recurrence and 15-year survival: an overview of the randomised trials. Lancet 2005; 365:1687-717.

4. Saphner T; Tormey DC; Gray R. Venous and arterial thrombosis in patients who received adjuvant therapy for breast cancer. J Clin Oncol 1991; 9:286-94.

5. Duggan C, Marriott K, Edwards R, Cuzick J. Inherited and acquired risk factors for venous thromboembolic disease among women taking tamoxifen to prevent breast cancer. $\mathrm{J}$ Clin Oncol 2003; 21:3588-93.

6. Bertelli G, Pronzato P, Amoroso D, Cusimano MP, Conte PF, Montagna G, Bertolini S, Rosso R. Adjuvant tamoxifen in primary breast cancer: influence on plasma lipids and antithrombin III levels. Breast Cancer Res Treat 1988; 12:307-10.

7. Love RR, Surawicz TS, Williams EC. Antithrombin III level, fibrinogen level, and platelet count changes with adjuvant tamoxifen therapy. Arch Intern Med 1992; 152:317-20.

8. Pemberton $\mathrm{KD}$; Melissari E; Kakkar VV The influence of tamoxifen in vivo on the main natural anticoagulants and fibrinolysis. Blood Coagul Fibrinolysis 1993; 4:935-42.

9. Braithwaite RS, Chlebowski RT, Lau J et al. Meta-analysis of vascular and neoplastic events associated with tamoxifen. J Gen Intern Med 2003; 18:937-47

10. Swerdlow AJ, Jones ME. Tamoxifen treatment for breast cancer and risk of endometrial cancer: a case-control study. J Natl Cancer Inst 2005; 97:375-84.

11. Curtis RE, Freedman DM, Sherman ME, Fraumeni JF Jr. Risk of malignant mixed mullerian tumors after tamoxifen therapy for breast cancer. J Natl Cancer Inst 2004; 96:70-4.

12. Machado F, Rodriguez JR, Leon JP, Rodriguez JR, Parrilla JJ, Abad L. Tamoxifen and endometrial cancer. Is screening necessary? A review of the literature. Eur J Gynaecol Oncol $2005 ; 26: 257-65$. 
13. Cohen I, Rosen DJ. Shapira J, et al. Endometrial changes with tamoxifen: comparison between tamoxifen-treated and nontreated asymptomatic, postmenopausal breast cancer patients. Gynecol Oncol 1994; 52:185-90.

14. Kedar RP', Bourne TH, Powles TJ, et al. Effects of tamoxifen on uterus and ovaries of postmenopausal women in a randomised breast cancer prevention trial. Lancet 1994; 343:1318-21.

15. Cecchini S, Ciatto S, Bonardi R, et al. Screening by ultrasonography for endometrial carcinoma in postmenopausal breast cancer patients under adjuvant tamoxifen. Gynecol Oncol 1996; 60:409-11.

16. Love CD, Muir BB, Scrimgeour JB, Leonard RC, Dillon P, Dixon JM. Investigation of endometrial abnormalities in asymptomatic women treated with tamoxifen and an evaluation of the role of endometrial screening. J Clin Oncol 1999; 17:2050-4.

17. Gerber B, Krause A, Muller H, et al. Effects of adjuvant tamoxifen on the endometrium in postmenopausal women with breast cancer: a prospective long-term study using transvaginal ultrasound. J Clin Oncol 2000; 18:3464-70.

18. Barakat RR, Gilewski TA, Almadrones L, et al. Effect of adjuvant tamoxifen on the endometrium in women with breast cancer: a prospective study using office endometrial biopsy. $J$ Clin Oncol 2000; 18:3459-63.

19. Bertelli $G$, Venturini $M$, Del Mastro $L$ et al. Tamoxifen and the endometrium: findings of pelvic ultrasound examination and endometrial biopsy in asymptomatic breast cancer patients. Breast Cancer Res Treat 1998; 47:41-6

20. Bertelli G, Valenzano M, Costantini S, Rissone R, Angiolini C, Signorini A, Gustavino C. Limited value of sonohysterography for endometrial screening in asymptomatic, postmenopausal patients treated with tamoxifen. Gynecol Oncol 2000; 78:275-7.

21. Khovidhunkit W, Shoback DM. Clinical effects of raloxifene hydrochloride in women. Ann Intern Med 1999; 130:431-9.

22. Shibutani S, Ravindernath A, Terashima I, Suzuki N, Laxmi YR, Kanno Y, Suzuki M, Apak TI, Sheng JJ, Duffel MW. Mechanism of lower genotoxicity of toremifene compared with tamoxifen. Cancer Res 2001; 61:3925-31.

23. Hachisuga $T$, Tsujioka $H$, Horiuchi $S$, Udou T, Emoto $M$, Kawarabayashi T. K-ras mutation in the endometrium of tamoxifen-treated breast cancer patients, with a comparison of tamoxifen and toremifene. Br J Cancer. 2005; 92:1098-103.

24. Bertelli G, Queirolo P, Vecchio S, Angiolini C, Bergaglio M, Del Mastro L, Signorini A, Valenzano $M$, Venturini $M$. Toremifene as a substitute for adjuvant tamoxifen in breast cancer patients. Anticancer Res 2000; 20:3659-61.

25. Powles TJ, Bourne T, Athanasiou S, et al. The effects of norethisterone on endometrial abnormalities identified by transvaginal ultrasound screening of healthy post-menopausal women on tamoxifen or placebo. Br J Cancer 1998; 78:272-5.

26. Bertelli G, Venturini M, Bergaglio M, Gustavino C, Rosso R, Valenzano M. Progestins and the endometrium in patients receiving tamoxifen. Br J Cancer 1999; 80:1114.

27. Gardner FJ, Konje JC, Abrams KR, et al. Endometrial protection from tamoxifen-stimulated changes by a levonorgestrel-releasing intrauterine system: a randomised controlled trial. Lancet 2000; 356:1711-7.

28. Hoda D, Perez DG, Loprinzi CL. Hot flashes in breast cancer survivors. Breast J 2003; 9:431-8.

29. Loprinzi CL, Michalak JC, Quella SK et al. Megestrol acetate for the prevention of hot flashes. N Engl J Med 1994; 331: 347-352. 
30. Bertelli $G$, Venturini $M$, Del Mastro $L$, Bergaglio $M$, Sismondi $P$, Biglia $N$, Venturini $S$, Porcile G, Pronzato $P$, Costantini $M$, Rosso $R$. Intramuscular depot medroxyprogesterone versus oral megestrol for the control of postmenopausal hot flashes in breast cancer patients: a randomized study. Ann Oncol 2002; 13:883-8.

31. Smith IE, Dowsett M. Drug therapy: Aromatase inhibitors in breast cancer. N Engl J Med $2003 ; 348: 2431-42$.

32. Jonat $\mathrm{W}$, Howell $\mathrm{A}$, Blomqvist $\mathrm{C}$, et al. A randomised trial comparing two doses of the new selective aromatase inhibitor anastrozole (Arimidex) with megestrol acetate in postmenopausal patients with advanced breast cancer. Eur J Cancer 1996; 32:404-12.

33. Buzdar AU, Jones SE, Vogel CL, Wolter J, Plourde P, Webster A. A phase III trial comparing anastrozole ( 1 and 10 milligrams), a potent and selective aromatase inhibitor, with megestrol acetate in postmenopausal women with advanced breast carcinoma. Arimidex Study Group. Cancer 1997; 79:730-9.

34. Dombernowsky P, Smith I, Falkson G, et al. Letrozole, a new oral aromatase inhibitor for advanced breast cancer: double-blind randomized trial showing a dose effect and improved efficacy and tolerability compared with megestrol acetate. J Clin Oncol 1998; 16:453-61.

35. Kaufmann M, Bajetta E, Dirix LY, et al. Exemestane is superior to megestrol acetate after tamoxifen failure in postmenopausal women with advanced breast cancer: results of a phase III randomized double-blind trial. The Exemestane Study Group. J Clin Oncol 2000; 18:1399-411.

36. Buzdar A, Douma J, Davidson N, et al. Phase III, multicenter, double-blind, randomized study of letrozole, an aromatase inhibilor, for advanced breast cancer versus megestrol acetate. J Clin Oncol 2001; 19:3357-66.

37. Nabholtz JM, Bonneterre J, Buzdar A, Robertson JF, Thurlimann B. Anastrozole (Arimidex) versus tamoxifen as first-line therapy for advanced breast cancer in postmenopausal women: survival analysis and updated safety results. Eur J Cancer 2003; 39:1684-9.

38. Bonneterre J, Thurlimann B, Robertson JF, et al. Anastrozole versus tamoxifen as first-line therapy for advanced breast cancer in 668 postmenopausal women: results of the Tamoxifen or Arimidex Randomized Group Efficacy and Tolerability study. J Clin Oncol 2000; 18:3748-57.

39. Mouridsen $\mathrm{H}$, Gershanovich $\mathrm{M}$, Sun $\mathrm{Y}$, et al. Phase III study of letrozole versus tamoxifen as first-line therapy of advanced breast cancer in postmenopausal women: analysis of survival and update of efficacy from the International Letrozole Breast Cancer Group. J Clin Oncol 2003; 21:2101-9.

40. Paridaens R, Therasse P, Dirix L, et al. First line hormonal treatment (HT) for metastatic breast cancer (MBC) with exemestane (E) or tamoxifen $(\mathrm{T})$ in postmenopausal patients (pts) - a randomized phase III trial of the EORTC brcast group. Proc Am Soc Clin Oncol 2004; 23:6 (Abstract 515).

41. Howell A, Cuzick J, Baum M, et al. Results of the ATAC (Arimidex, Tamoxifen, Alone or in Combination) trial after completion of 5 years' adjuvant treatment for breast cancer. Lancet 2005; 365:60-2.

42. Thurlimann B, Keshaviah A, Coates AS, et al. A comparison of letrozole and tamoxifen in postmenopausal women with early breast cancer. N Engl J Med 2005; 353:2747-57.

43. Jakesz R, Jonat W, Gnant $M$, et al. Switching of postmenopausal women with endocrineresponsive early breast cancer to anastrozole after 2 years' adjuvant tamoxifen: combined results of ABCSG trial 8 and ARNO 95 trial. Lancet 2005; 366:455-62. 
44. Boceardo F, Rubagotti A. Puntoni $M$, et al. Switching to anastrozole versus continued tamoxifen treatment of early breast cancer: preliminary results of the ltalian Tamoxifen Anastrozole Trial. J Clin Oncol 2005: 23:5138-47.

45. Cioss PE, Ingle $I N$, Martino $S$, et al. Randomized trial of letrozole following tamoxifen as extended adjuvant therapy in receptor-positive breast cancer: updated findings from NCIC CTC MA.17. J Natl Cancer Inst 2005; 97:1262-71.

46. Love RR. Maress RB, Barden HS, et al. Effects of tamoxifen on bone mineral density in postmenopausal women with breast cancer. N Engl J Med 1992; 326:852-856.

47. Coombes RC, Iall E, Gibson LJ, et al. A randomized trial of exemestane after two to three years of tamoxifen therapy in postmenopausal women with primary breast cancer. $\mathrm{N}$ Engl $J$ Med 2004; 350:1081-92

48. Kloke $\mathrm{O}$, Klaassen $\mathrm{U}$, Oherhoff $\mathrm{C}$, et al. Maintenance treatment with medroxyprogesterone acetate in patients with advanced breast cancer responding to chemotherapy: results of a randomized trial. Essen Breast Cancer Study Group. Breast Cancer Res Treat 1999; 55:51-9.

49. Bertelli $\mathrm{G}$, Garrone $\mathrm{O}$, Bertolotti $\mathrm{L}$, et al. Maintenance hormone therapy with letrozole after first-line chemotherapy for advanced breast cancer. Oncology 2005; 68:364-70.

50. Thurlimann B, Paridaens R, Serin D, et al. Third-line hormonal treatment with exemestane in postmenopausal patients with advanced breast cancer progressing on aminogluthetimide: a phase II multicentre multinational study. Eur J Cancer 1997; 33:1767-73.

51. Lonning PE, Bajetta E, Murray R et al. Activity of exemestane (Aromasin(B)) in metastatic breast cancer after failure of nonsteroidal aromatase inhibitors: a phase II trial. J Clin Oncol 2000; 18:2234-44.

52. Bertelli $\mathrm{G}$, Garrone $\mathrm{O}$, Merlano $\mathrm{M}$ et al. Sequential use of aromatase inactivators and inhibitors in advanced breast cancer. Oncology 2005; 69:471-477.

53. Howell A, Robertson JFR, Quaresma Albano J et al. Fulvestrant, formerly ICI 182,780, is as effective as anastrozole in postmenopausal women with advanced breast cancer progressing after prior endocrine treatment. J Clin Oncol 2002; 20: 3395-403. 


\title{
Chapter 2
}

\author{
Tamoxifen and the endometrium: \\ findings of pelvic ultrasound examination \\ and endometrial biopsy in asymptomatic \\ breast cancer patients
}

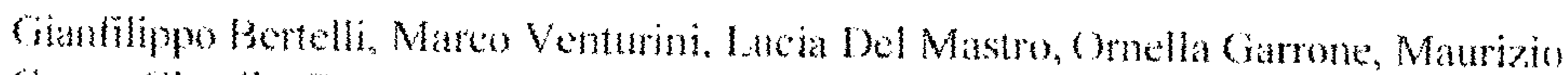
Cosso, Clatudo Gustavino, Lnzo Cusimano. Tiziana Cuido, Guido Ninolo', and Ricerato Resso

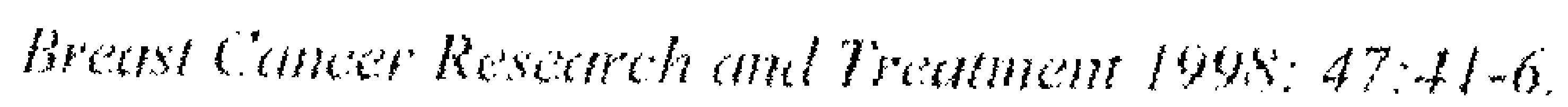




\section{Summary}

The need for endometrial surveillance in breast cancer patients undergoing adjuvant treatment with tamoxifen is still controversial. In this study, 164 asymptomatic breast cancer patients (110 on treatment with tamoxifen, $20 \mathrm{mg} / \mathrm{day}$, and 54 controls) were examined with pelvic ultrasound and endometrial biopsy. No differences in ultrasound and biopsy findings were observed in the pre- and perimenopausal group between patients treated with tamoxifen and controks. Postmenopausal patients on tamoxifen had a significantly thicker endometrium (mean $\pm \mathrm{SD}, 7.2 \pm 8.5 \mathrm{vs} .1 .5 \pm 4.3 \mathrm{~mm}, \mathrm{p}=0.00002$ ) and significantly larger uterine volume (mean $\pm \mathrm{SD}, 63.2$ \pm 39.9 vs. $43.7 \pm 38.8 \mathrm{~cm}^{3}, p=0.0001$ ) than controls. Fifty-four percent of patients on tamoxiten had an endometrial thickness $\geq 5 \mathrm{~mm}$, often with multiple irregular sonolucencies suggesting the presence of cysts. Ultrasound findings, however, did not correlate with the presence of endometrial abnormalities on biopsy, and no endometrial cancer or atypical hyperplasia were found. This lack of correlation makes questionable the use of routine sonography in asymptomatic breast cancer patients on tamoxifen. Obtaining routine endometrial samples, on the other hand, may be difficult in some patients because of cervical stenosis or refusal. Until the benefits of endometrial surveillance will be proved, asymptomatic patients should not be submitted routinely to ultrasound examination or biopsy, but encouraged to report promptly any abnormal vaginal bleeding.

\section{Introduction}

The antiestrogen tamoxifen has been widely used for the adjuvant treatment of breast cancer in the last three decades [1]. Although generally considered a safe drug, several reports of a link with the development of endometrial cancer have appeared in the literature, raising concerns over the appropriate management of patients during tamoxifen treatment [2-4]. There is little doubt that any patient taking tamoxifen and reporting abnormal vaginal bleeding should be promptly evaluated to exclude the presence of an endometrial malignancy. The opportunity of a program of endometrial surveillance in asymptomatic patients, however, remains controversial. Considering the large number of women taking adjuvant tamoxifen, and the potentially negative impact of invasive examinations on compliance to the treatment, a potential program of endometrial surveillance in asymptomatic patients should be based on simple and acceptable procedures with a demonstrable cost/benefit ratio.

Transvaginal ultrasound examination is a useful diagnostic tool for endometrial abnormalities [5]; the transabdominal technique, however, is less invasive and could be more suitable in this setting [6]. Office endometrial biopsies have also been used to monitor the endometrium of breast cancer patients on tamoxifen [7]. A recent inquiry suggested that, even outside clinical studies, an increasing number of breast cancer patients are being submitted to routine ultrasound examination and/or biopsy [8]. More scientific evidence, however, would be needed to justify this behaviour. In the present study, we report the findings of pelvic ultrasound examination and endometrial sampling in asymptomatic breast cancer patients receiving adjuvant tamoxifen or candidates to start treatment with this drug.

\section{Patients and methods}

Patients were consecutively recruited among women receiving from at least one month tamoxifen as adjuvant hormone therapy after surgical treatment for stage I-II breast 
cancer, and attending our outpatient clinic. Eligibility criteria were: no prior hysterectomy, no gynecological symptoms, no evidence of distant metastases or local relapse. Patients with similar characteristics, but who had just been prescribed adjuvant tamoxifen after surgery and had not yet started the treatment, formed the control group. An informed consent for participation in the study was obtained from all subjects, according to the requirements of the local Ethical Committee.

Pelvic ultrasonography was performed using a $3.5 \mathrm{MHz}$ AV 590 (Esaote Biomedica) system. The longitudinal (L), transverse (T), and anteroposterior (AP) diameters of the uterus were measured through the full bladder using digital calipers: endometrial thickness was measured at the thickest part of the longitudinal plane. Uterine volume (V) was calculated using the formula $V=L \times T \times A P \times 0.5$. Endometrial samples were obtained with a disposable Perma curette and sent for histological examination to one pathologist (GN) who was blinded to the treatment of the patient. In postmenopausal patients, a negative finding was defined as atrophic endometrium or insufficient tissue for histological diagnosis; a positive finding was any other pattern of tissue sufficient for histological diagnosis.

The statistical analyses employed for the comparison of groups were the chi-square exact test and the Mann-Whitney U-test as appropriate. To assess the association between endometrial thickness or positive endometrial biopsy and tamoxifen therapy, while adjusting for the confounding effect of other factors (age, duration of menopause, body mass index, parity, or gravidity), a multivariate logistic model was used.

\section{Results}

One hundred and sixty-four consecutive, gynecologically asymptomatic early breast cancer patients were enrolled in the study from February 1994 to July 1996. One hundred and ten were receiving adjuvant tamoxifen at the daily dose of $20 \mathrm{mg}$; the median duration of tamoxifen treatment at the time of entry into the study was 15 months, with a range of 1 month to 7 years. Fifty-four patients, forming the control group, underwent the examinations just before starting treatment with adjuvant tamoxifen.

The main characteristics of patients in the two groups are shown in Table 1. The tamoxifen and control groups were well balanced in terms of age, duration of menopause, parity, gravidity and body mass index. Due to the fact that until a few years ago. premenopausal women were not often prescribed adjuvant tamoxifen in our institution, a significantly larger number of patients already on tamoxifen were postmenopausal as compared to controls.

Overall, endometrial thickness measured by pelvic ultrasound significantly increased in the tamoxifen group as compared to the control group (mean $\pm \mathrm{SD}, 7 \pm 8.4 \mathrm{~mm}$ vs. $2.9 \pm 5.3 \mathrm{~mm}, \mathrm{p}<0.01)$. Mean uterine volume was $73.4 \pm 53.8 \mathrm{~cm}^{3}$ in tamoxifen-treated patients and $62.6 \pm 47.1 \mathrm{~cm}^{3}$ in control patients ( $\mathrm{p}=$ not significant).

Table 2 shows the results of pelvic ultrasound in postmenopausal patients: those treated with tamoxifen had a significantly thicker endometrium $(p=0.00002)$ and significantly larger uterine volume $(\mathrm{p}=0.0005)$ than control patients. As expected in normal postmenopausal women, the endometrium was not visible or measured less than $5 \mathrm{~mm}$ in the vast majority ( $88.2 \%)$ of control patients. On the other hand, more than half $(53.9 \%)$ of the patients receiving tamoxifen had sonographic evidence of hyperechoic, thickened 
TABLE 1

Characteristics of patients.

\begin{tabular}{lll}
\hline & Tamoxifen & Control \\
\hline $\begin{array}{l}\text { No. of patients } \\
\text { Menopausal status: }\end{array}$ & $110(\%)$ & $54(\%)$ \\
Pre-menopausal & $7(12.7)$ & $13(24)^{*}$ \\
Peri-menopausal & $14(6.4)$ & $7(13)$ \\
Post- menopausaI & $89(80.9)$ & $34(63)^{* *}$ \\
Median duration of menopause: & & \\
Months & 138 & 157 \\
Range & $15-431$ & $20-552$ \\
Age (years): & & \\
Median & 60 & 57 \\
Range & $39-84$ & $37-86$ \\
Parity: & $10(9.1)$ & $9(16.7)$ \\
0 & $97(88.2)$ & $43(79.6)$ \\
1-3 & $3(2.7)$ & $2(3.7)$ \\
$\geq 4$ & $10(9.1)$ & $9(16.7)$ \\
Gravidity: & $89(80.9)$ & $41(75.9)$ \\
0 & $11(10)$ & $4(7.4)$ \\
1-3 & & \\
$\geq 4$ & 25.1 & 24.7 \\
Body mass index: & $19.4-48.9$ & $17-40.8$ \\
Median & & \\
Range & & \\
\hline
\end{tabular}

$* \quad p<0.001 ; * * \quad p<0.01$.

All other differences are not statistically significant. Body Mass Index was calculated as the ratio of weight in kilograms to squared height in meters.

TABLE 2

Pelvic ultrasound findings in postmenopausal patients.

\begin{tabular}{lll}
\hline & $\begin{array}{l}\text { Tamoxifen } \\
n(\%)\end{array}$ & $\begin{array}{l}\text { Control } \\
\mathrm{n}(\%)\end{array}$ \\
\hline $\begin{array}{l}\text { No. of patients } \\
\text { Endometrial thickness (ET): }\end{array}$ & $89(72.3)$ & $34(27.7)$ \\
$\quad$ Mean ET $(\mathrm{mm}) \pm$ SD & $7.2 \pm 8.5$ & $1.5 \pm 4.3^{*}$ \\
ET $<5$ & $41(46.1)$ & $30(88.2)$ \\
ET 5 -7 & $9(10.1)$ & $0 * *$ \\
ET $>7$ & $39(43.8)$ & $4(11.8)$ \\
Uterine volume (UV): & & $43.7 \pm 38.8^{* * *}$ \\
$\quad$ Mean UV $\left(\mathrm{cm}^{3}\right) \pm$ SD & $63.2 \pm 39.9$ & \\
\hline
\end{tabular}

* $p=0.00002 ; * * \quad p=0.0001 ; * * * \quad p=0.015$

endometrium, often with multiple sonolucent areas suggesting the presence of cysts. Multivariate regression analysis demonstrated that tamoxifen was the only significant independent factor predicting for endometrial thickness. In the tamoxifen group, no significant correlation between endometrial thickness and duration of tamoxifen was evident. 
In the pre- and perimenopausal patients, on the other hand, no significant differences appeared between tamoxifen-treated and untreated patients (data not shown).

Endometrial samples were obtained in 124 patients $(75.6 \%)$. Nine patients $(4$ in the tamoxifen group and 5 control patients) refused the procedure, and in 31 cases ( 21 in the tamoxifen group and 10 in control patients) cervical stenosis precluded the insertion of the Perma curette. No abnormal findings were evident in histological examination of endometrial tissue obtained from pre- or perimenopausal patients, both in the tamoxifen and control group. In the 91 postmenopausal patients for whom endometrial samples were available, positive findings were present in 21 patients $(31.3 \%)$ in the tamoxifen group and in 5 patients $(20.8 \%)$ in the control group $(\mathrm{p}=$ not significant). Positive findings included two cases of endometrial hyperplasia without atypia (one in each group). In three of the positive samples from patients receiving tamoxifen, and one in the control group, hypertrophy of the sub-endometrial stroma was evident. No atypical endometrial hyperplasia or endometrial cancer was found in either group. In the patients receiving tamoxifen, no significant correlations between positive histological findings and endometrial thickness, uterine volume, body mass index, age, menopausal age, or duration of tamoxifen treatment were observed.

All patients underwent a gynecologic examination during which a cervical smear was taken in the standard fashion with an Ayer's spatula and stained by the Papanicolaou technique. This allowed diagnosis of two high-grade squamous intraepithelial lesions of the cervix (one in tamoxifen-treated patients and one in the control group).

\section{Discussion}

Following the demonstration of its benefits, an increasing number of pre- and postmenopausal breast cancer patients, including many with good prognosis, are being treated with adjuvant tamoxifen [9]. The balance between the beneficial effects of tamoxifen and its side effects is generally considered very good. Beside the antiestrogen activity, tamoxifen has some agonist estrogenic properties, which may actually have a positive impact on bone resorption [10] and cardiovascular risk factors [11]. Not surprisingly, since unopposed estrogen stimulation of the endometrium is known to be carcinogenic [12], an association between tamoxifen treatment and endometrial cancer risk has also emerged. Patients receiving tamoxifen should be aware of this risk and instructed to seek gynecologic consultation for any instance of abnormal vaginal bleeding. No screening recommendations, however, have been so far formulated for asymptomatic tamoxifen-treated patients. The results obtained by Kedar et al. [13] in a group of healthy women, participating in the pilot tamoxifen prevention trial conducted in the UK, suggested that utrasonography can be used to detect the subjects who should unJergo endonetrial sampling. In the Kedar study, women with a histological abnormality rad a significantly thicker endometrium, and the predictive value of an endometrial hickness $\geq 8 \mathrm{~mm}$ for atypical hyperplasia or polyps was $100 \%$. However, a few recent studies in gynecologically asymptomatic breast cancer patients receiving tamoxifen lave reported contrasting results. Lahti et al. [14] compared 51 postmenopausal patients eceiving $20-40 \mathrm{mg}$ of tamoxifen, as adjuvant or for recurrence, with 52 control breast :ancer patients. In the tamoxifen group, the endometrium was significantly thicker and he uterine volume larger on transvaginal sonography, but - although there was a sig- 
nificantly higher occurrence of endometrial polyps - histological data obtained by hysteroscopy did not demonstrate an increased occurrence of precancerous lesions such as atypical hyperplasia. Cohen et al. [15] observed, again with transvaginal sonography, a similar increase of endometrial thickness in 93 postmenopausal breast cancer patients treated with 20-30 mg of tamoxifen, as compared to 20 untreated patients. Suction curettage or hysteroscopy were used to obtain endometrial samples. Positive endometrial findings as a whole (i.e., any pattern of tissue obtained by endometrial sampling, which was sufficient for histological evaluation) were more frequent in the tamoxifen group than in the control patients ( 35 vs. $20 \%$. respectively); this included three endometrial cancers and five endometrial polyps in the tamoxifen group versus none in the control group. In another study by Rayter et al. [16], 49 pre-and postmenopausal patients who had taken tamoxifen for at least 3 years ( 13 of whom had stopped the treatment at the time of the study) and 45 control breast cancer patients who had never received hormone therapy underwent pelvic sonography, cervical smears, and endometrial sampling. Although the study suggested that endometrial thickness was greater in tamoxifentreated patients than in controls, there was no evidence of an increase in preneoplastic changes.

In our study, a significant increase in endometrial thickness and uterine volume was found in a group of postmenopausal breast cancer patients receiving adjuvant tamoxifen, 20 mg daily, as compared to patients who were examined just before starting the treatment. Although the majority of patients on tamoxifen had an endometrial thickness $\geq 5 \mathrm{~mm}$, only two cases of endometrial hyperplasia without atypia (one in patients on tamoxifen, and one in controls) were found on biopsy. Taken together, these data suggest that for many postmenopausal breast cancer patients treated with tamoxifen, the finding of a thickened endometrium on ultrasound examination is not necessarily associated with endometrial pathology on biopsy. Even increasing the threshold for abnormal endometrial thickness in postmenopause from 5 to $8 \mathrm{~mm}$, as suggested by the results of Kedar et al.,43\% of the asymptomatic patients in our study would have had an indication for biopsy. Such paradoxical sonographic finding of thickened, heterogeneous endometrium, often with bizarre-looking irregular sonolucencies, in patients who have normally atrophic endometrium on biopsy has been interpreted to be actually subendometrial in origin $[17,18]$. This seems to be confirmed by the recent results of a study of our institute, which investigated the effect of $20 \mathrm{mg}$ tamoxifen on histology and flow cytometric DNA content distribution of endometrial samples [19]. Although tamoxifen and tamoxifen duration were associated with»an increase in the risk of proliferation, no epithelial hy-perplasia was observed on biopsy. Tamoxifen induced hyperproliferation, in fact, appeared to be mediated by the stromal component of the material obtained by endometrial biopsy.

In conclusion, the results of ultrasound investigation of the endometrium in asymptomatic breast cancer patients receiving tamoxifen should be interpreted with caution. An over diagnosis of endometrial abnormalities, and the consequent unnecessary invasive diagnostic procedures, could discourage some patients from continuing the adjuvant treatment with tamoxifen, putting them at increased risk for a relapsing breast cancer. Other imaging techniques could be used to obtain more specific information on endometrial changes in patients receiving tamoxifen, when ultrasound abnormalities are found. Transvaginal color Doppler sonography was found helpful to exclude endometrial pathology in $6 / 7$ patients with thick and cystic endometrium on conventional 
lography [20]; in another report [21], color Doppler ultrasound showed a signifiitly decreased mean resistance index (RI) in 20 patients with thickened endometrium, compared with 25 having normal endometrium appearance. Eight patients in the first up were found to have endometrial polyps; in $6 / 8$ cases, the RI was $<0.4$ [21]. In itrast with these promising results, however, another investigation using Doppler asonography in 39 patients treated with tamoxifen failed to find a correlation besen uterine artery pulsatility index values and endometrial widths [22]. The instilla$\gamma$ of saline solution into the endometrial cavity during transvaginal ultrasonography nohysterography) is an additional, potentially useful diagnostic method; in a recent ort [23], sonohysterography was performed in 20 patients with cystic thickened ennetrium, allowing the detection of 8 endometrial polyps. The results of magnetic onance (MR) imaging have also been described in a group of 33 patients treated with toxifen [23]; two uterine imaging patterns were noted, one of which (heterogeneous lometrial signal intensity on T2-weighted images) was present in 17/33 patients and 12 cases was associated with polyps. Clearly, additional studies are urgently needed :larify the role of the above mentioned techniques, but as far as conventional ultrand is concerned, there is no evidence that patients receiving adjuvant tamoxifen Id benefit from a screening program based on this method alone. Our study also gests that in unselected patients, the attempt to obtain systematically an endometrial Iple with a Perma curette or other techniques not requiring cervical dilatation will be ited, in a significant proportion of cases, by cervical stenosis or patient's refusal. il the benefits of endometrial surveillance will be proved, asymptomatic breast canpatients on adjuvant tamoxifen should not be submitted routinely to ultrasound exnation or biopsy, but encouraged to report promptly any abnormal vaginal bleeding.

\section{ferences}

Jaiyesimi IA, Buzdar AU, Decker DA, Hortobagyi GN: Use of tamoxifen for breast cancer: Twenty-eight years later. J Clin Oncol 13:513-529,1995

Fisher B, Costantino JP, Redmond CK. Fisher ER, Wicker-ham DL, Cronin WM, and other NASBP Contributors: Endometrial cancer in tamoxifen-treated breast cancer patients: findings from the National Surgical Adjuyant Breast and Bowel Project (NSABP) B-14. J Nat) Cancer Inst 86: 527-537,1994

Jordan VC, Morrow M: Should clinicians be worried about the carcinogenic potential of tamoxifen? Eur J Cancer 30A: 1714-1721, 1994

Neven P, De Muylder X, Van Belle Y, Campo R, Vanderick G: Tamoxifen and the uterus. Br Med J 309:1313-1314,1994

Hulka CA, Hall DA, McCarthy K, Simeone JF: Endometrial polyps, hyperplasia. and carcinoma in postmenopausal women: differentiation with endovaginal sonography. Radiology 191: 755-758,1994

Ciatto S, Cecchini S, Bonardi R, Grazzini G: Ultrasonography surveillance of endometrium in breast cancer patients on adjuvant tamoxifen. Lancet $344: 60,1994$

Barakat RR, Gilewski TA, Saigo PE, Almadrones L, Tyson P, Norton L, Hoskins WJ: The effect of adjuvant tamoxifen on the endometrium in women with breast cancer: an interim analysis of a prospective study. Proc Am Soc Clin Oncol 14: 227,1995 (abs) 
8. Schwartz PE: Gynecologic surveillance of women taking tamoxifen. Conn Med 58: 515521,1994

9. Early Breast Cancer Trialists' Collaborative Group: Systemic treatment of early breast cancer by hormonal, cytotoxic, or immune therapy. 133 randomised trials involving 31.000 recurrences and 24.000 deaths among 75.000 women. Lancet 339:1-15, 71-85, 1992

10. Love RR, Mazess RB, Barden HS, Epstein S, Newcomb PA, Jordan VC, Carbone PP. DeMets DL: Effects of tamoxifen on bone mineral density in postmenopausal women with breast cancer. $N$ Engl J Med 326: 852-856, 1992

11. Love RR, Wiebe DA, Feyzi JM, Newcomb PA, Chappell RJ: Effects of tamoxifen on cardiovascular risk factors in postmenopausal women after 6 years of treatment. J Natl Cancer Inst 86:1534-1539,1994

12. Ziel HK: Estrogen's role in endometrial cancer. Obstet Gynecol 60: 509-515,1982

13. Kedar RP, Bourne TH, Powles TJ, Collins WP, Ashley SE, Cosgrove DO, Campbell S; Effects of tamoxifen on uterus and ovaries of postmenopausal women in a randomised breast cancer prevention trial. Lancet 343:1318-1321,1994

14. Lahti E, Blanco G, Kauppila A, Apaja-Sarkkinen M, Taski-nen PJ, Laatikainen T: Endometrial changes in postmenopausal breast cancer patients receiving tamoxifen. Obstet Gynecol 81: 660-664,1993

15. Cohen I, Rosen DJD, Shapira J, Cordoba M, Gilboa S, Altaras MM, Yigael D, Beyth Y: Endometrial changes with tamoxifen: comparison between treated and non-treated asymptomatic postmenopausal breast cancer patients. Gynecol Oncol 52:185-190,1994

16. Rayter Z, Gazet JC, Sheperd J, Trott PA, Fisher C, Svensson WE, Ford HT, Hern RA: Gynecological cytology and pelvic ultrasonography in patients with breast cancer taking tamoxifen compared with controls. Eur J Surg Oncol 20:134-140,1994

17. Bornstein J, Auslender R, Pascal B, Gutterman E, Isakov D, Abramovici H: Diagnostic pitfalls of ultrasonographic uterine screening in women treated with tamoxifen. J Reprod Med 39: 674-678,1994

18. Goldstein S: Unusual ultrasonographic appearance of the uterus in patients receiving tamoxifen. Am J Obstet Gynecol 170: 447-451,1994

19. Decensi A, Fontana V, Bruno S, Gustavino C, Gatteschi B, Costa A: Effect of tamoxifen on endometrial proliferation. J Clin Oncol 14: 434-440,1996

20. Aleem FA, Predanic M: Endometrial changes in patients on tamoxifen. Lancet 346: 12921293,1995

21. Achiron R. Lipitz S, Sivan E, Goldenberg M, Horovitz A, Frenkel Y, Mashiach S: Changes mimicking endometrial neoplasia in postmenopausal tamoxifen-treated women with breast cancer: a transvaginal Doppler study. Ultrasound Obstet Gynecol 6:116-120,1995

22. Tepper R, Cohen I, Altaras M, Shapira J, Cordoba M, Dror Y, Beyth Y: Doppler flow evaluation of pathologic endometrial conditions in postmenopausal breast cancer patients treated with tamoxifen. J Ultrasound Med 13: 635-640,1994

23. Achiron R, Lipitz S, Sivan E, Goldenberg M, Mahiach S: Sonohysterography for ultrasonograhic evaluation of tamoxifen associated cystic thickened endometrium. J Ultrasound Med 14: 685-688, 1995

24. Ascher SM, Johnson JC, Barnes WA, Bae CJ, Patt RH, Zeman RK: MR imaging appearance of the uterus in postmenopausal women receiving tamoxifen therapy for breast cancer: histopathologic correlation. Radiology 200:105-110, 1996 


\title{
Chapter 3
}

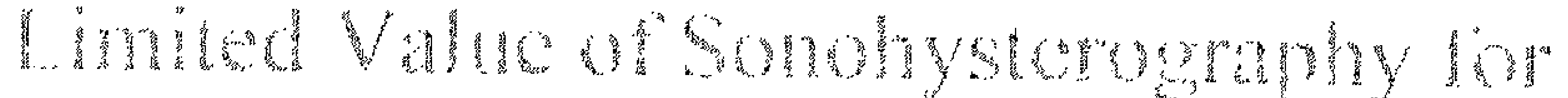

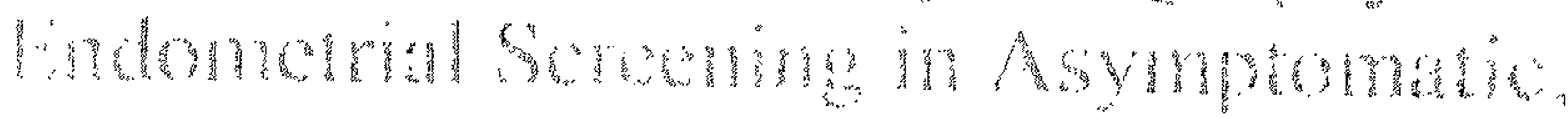

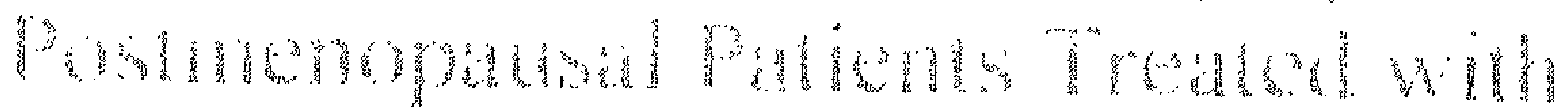

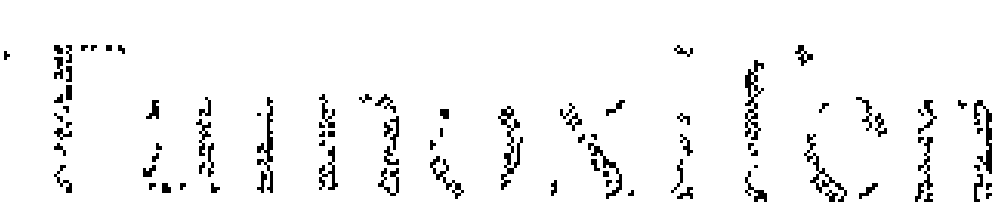

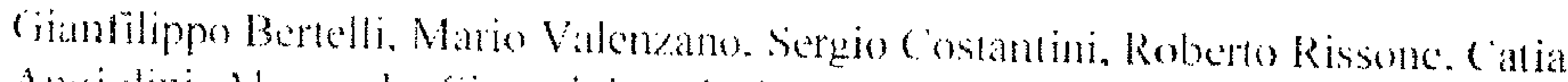

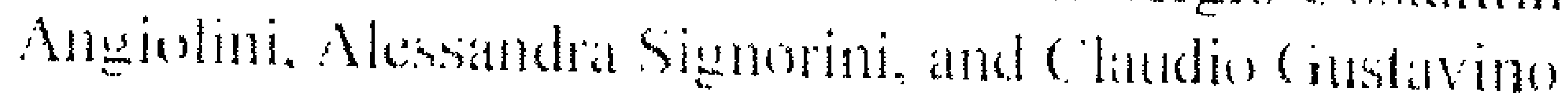

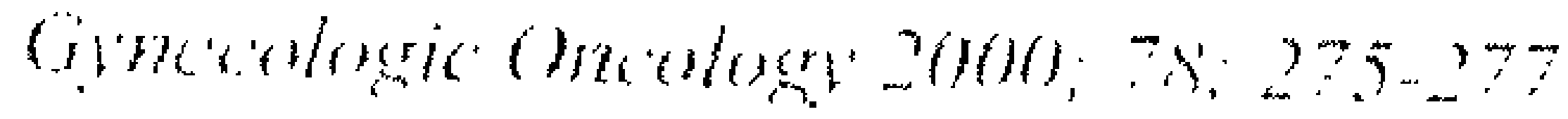




\section{Summary}

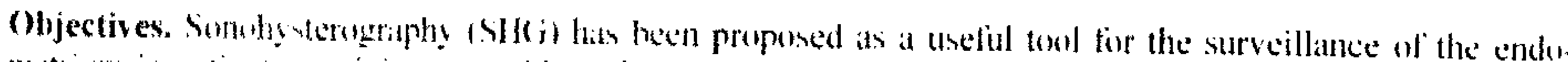

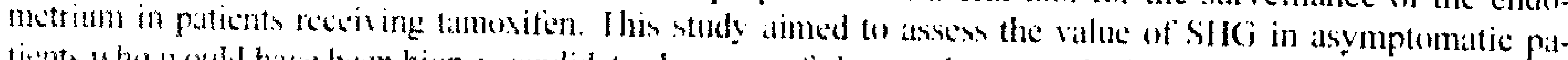

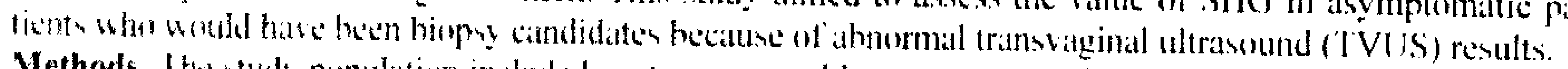

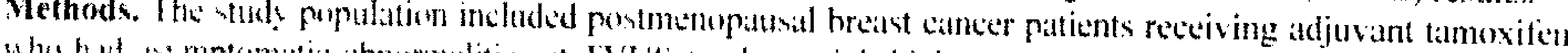

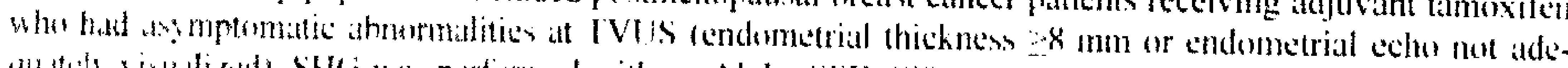

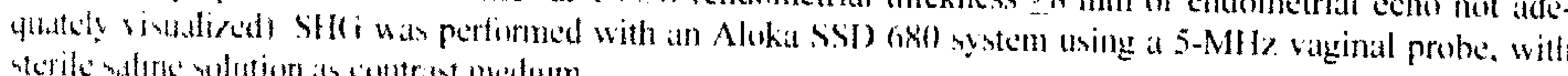

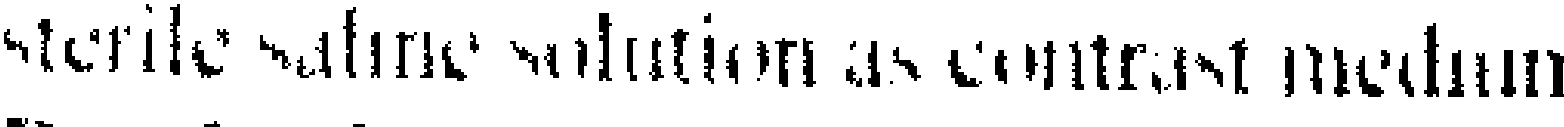

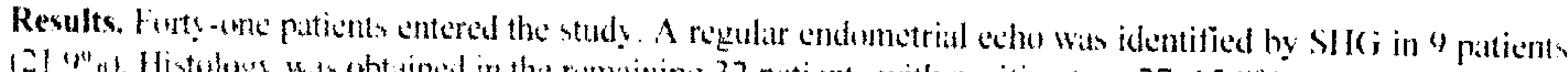

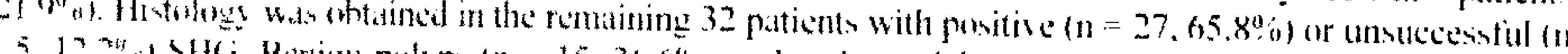

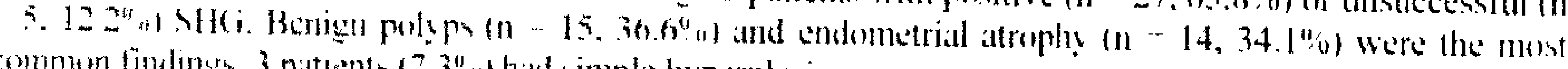

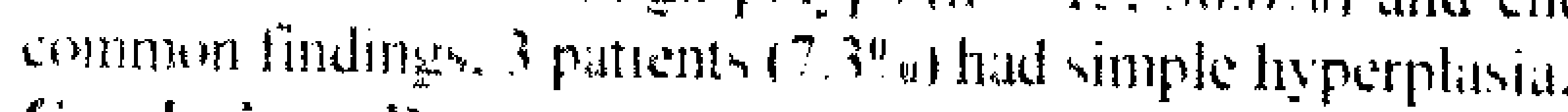

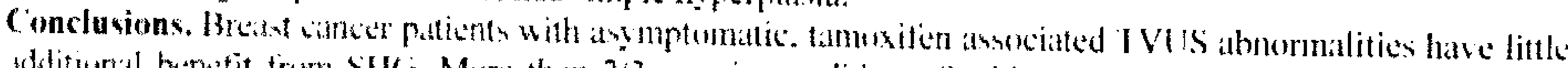

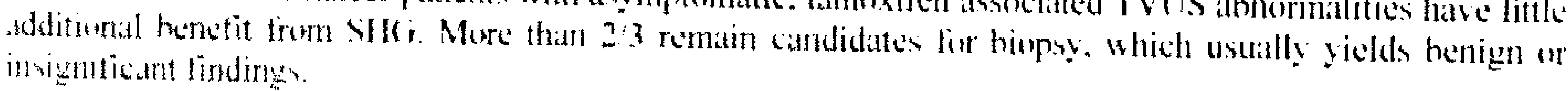

\section{Introduction}

Transvaginal ultrasonography is widely used in the workup for suspected endometrial pathology in postmenopausal women, where its value resides especially in a negative result: in the case of abnormal findings, additional testing is required to obtain a diagnosis [1]. Breast cancer patients receiving adjuvant tamoxifen may be considered at increased risk for endometrial cancer $[2,3]$ and are often very anxious about the possibility of developing a second tumor: thus, there has been much interest in recent years in ultrasound monitoring of the endometrium in this population. Following a number of reports which showed an unexpectedly high incidence of false-positive findings associated with tamoxifen use [4-7], however, most investigators now agree that ultrasound screening should not be encouraged in asymptomatic women on adjuvant tamoxifen [8.9].

Sonohysterography (SHG), which allows a better visualization of the endometrium by instilling a small volume of sterile saline into the uterine cavity before transvaginal ultrasound (TVUS), has shown promise in reducing the false-positive rate of ultrasound in tamoxifen-treated patients [10-12]. Based on these reports, we have studied with SHG a group of asymptomatic, postmenopausal breast cancer patients receiving adjuvant tamoxifen who had abnormal TVUS results.

\section{Patients and Methods}

Postmenopatusal, gynecologically asymptomatic breast cancer patients who attended our outpatient medical oncology clinic during treatment with adjuvant tamoxifen $(20$ nig/day) and had ahnormal routinc IV IS results were eligible to participate in the study, after providing intormed consent. IVUS was performed with an Aloka SSD 680 system using a 5-MHe vaginal prote (Aloka, Tokyo, Japati). Anteroposterior measurements of endonetrial thickness were obtained from the long-axis view, recording the maximum width between the lines separating the hyperechogenic endometrium from 
the myometrium. TVUS was defined as abnormal if a thickened endometrium $(\geq 8 \mathrm{~mm})$ was found or if the endometrial echo was not adequately visualized. SHG was performed with the same ultrasound system using sterile saline solution as contrast medium following the technique described by Tepper et al. [10]. In the case of negative SHG findings, i.e., when a thin endometrial echo without evidence of polyps or focal changes was identified, no further procedures were performed and the patient was followed up clinically. In all other instances, including a failure to perform SHG, patients were referred for hysteroscopy, and endometrial samples were sent for pathological examination. Polyps were removed and examined entirely.

\section{Results}

Forty-one postmenopausal, asymptomatic breast cancer patients receiving adjuvant tamoxifen ( $20 \mathrm{mg} /$ day) entered the study because TVUS had shown thickened $(\geq 8 \mathrm{~mm}$, $\mathrm{n}=39$ patients; median endometrial thickness $13 \mathrm{~mm}$, range 8-25) or inadequately visualized endometrium $(n=2)$. The median age of patients was 64 years (range 47-80); median time on treatment with tamoxifen was 26 months (range 5-84).

SHG was unsuccessful in 5 patients $(12.2 \%)$ due to cervical stenosis. A thin, regular endometrial echo was identified by SHG in 9 patients $(21.9 \%)$, who therefore did not undergo further evaluations. The presence of polyps was suggested by SHG in 19 patients $(46.3 \%)$, diffuse endometrial thickening in $2(4.9 \%)$, and focal endometrial thickening in $6(14.6 \%)$. Thirty-two patients with unsuccessful SHG or positive findings underwent hysteroscopy $(n=28,87.5 \%)$ or dilatation and curettage $(n=4,12.5 \%)$ to obtain histology. Table 1 summarizes the pathological findings and the correlation with SHG appearance. Benign polyps and atrophic endometrium were the more common diagnoses.

TABLE 1

Results of sonohysterography and correlation with pathological findings in 41 asymptomatic patients with endometrial thickening $(\geq 8 \mathrm{~mm})$ at TVUS during adjuvant Tamoxifen.

\begin{tabular}{lclll}
\hline & \multicolumn{4}{l}{ Pathology results } \\
\hline SHG diagnosis & No.of patients (\%) & Atrophic & Hyperplastic & Polyp \\
Polyp & $19(46.3)$ & 5 & 1 & 13 \\
Focal thickening & $6(14.6)$ & 4 & 1 & 1 \\
Diffuse thickening & $2(4.9)$ & 1 & 1 & 0 \\
Failcd & $5(12.2)$ & 4 & 0 & 1 \\
Normal & $9(21.9)$ & - & - & -
\end{tabular}

Note. Endometrial samples were not obtained in nine patients who had normal SHG results (thin endometrial echo without focal changes).

Only three patients had evidence of simple hyperplasia; of these, SHG had diagnosed a polyp in the first patient, focal thickening in the second, and diffuse thickening in the third. Fifteen polyps (13 previously identified by SHG, 1 found by hysteroscopy in a patient with failed SHG, and 1 found by hysteroscopy in a patient whose SHG had diagnosed a focal endometrial thickening) were resected. All polyps were found to be benign on pathological examination and were surrounded by atrophic endometrium; 4 
were common endometrial polyps, 8 were characterized by dense stroma, and 3 by cystically dilated glands with atrophic epithelium.

\section{Discussion}

Most postmenopausal patients receiving tamoxifen show sonographic abnormalities of the endometrium: however, the most common finding on histology in asymptomatic subjects is an atrophic epithelium [7. 8]. The discrepancy is probably due to specific tamoxifen-associated subepithelial changes, consisting of cystically dilated glands surrounded by dense stroma [13]: benign polyps, often characterized by the same pattern of cystic glands and stromal condensation. are also very common in patients receiving tamoxifen [14]. This results in an hyperechogenic. bizarre appearance of the endometrium at ultrasound, which limits its usefulness as a screening test in this population. Sonohysterography, which has been reported to enhance the specificity of TVUS [10.11], was used in our study in asymptomatic, postmenopausal tamoxifentreated patients who had abnormal TVUS results. With the aim of decreasing the number of unnecessary biopsies, we chose a cutoff of $8 \mathrm{~mm}$ to define abnormal endometrial thickening, instead of the standard $5 \mathrm{~mm}$. Despite this, only $21.9 \%$ of the patients had normal SHG results and avoided the biopsy. This can be compared with the findings in a similar study by Schwartz et al. [15], where patients were triaged for hysteroscopy with dilatation and curettage. blind biopsy, or no further procedure, based on the sequential use of TVUS and SHG: only $9 / 30(30 \%)$ of the patients undergoing SHG after an abnormal or unsuccessful TVUS avoided invasive procedures.

As in other studies. we found that SHG allows the identification of polyps, which would be otherwise missed by unenhanced TVUS. However, there is currently no evidence that, in asymptomatic patients under adjuvant tamoxifen, the identification and resection of endometrial polyps may be of benefit. Interestingly, a recent genomic study of tamoxifen-associated polyps suggests that they do not represent early steps in the process leading to malignancy $[16]$.

Patients who report abnormal vaginal bleeding during treatment with adjuvant tamoxifen must be promptly and thoroughly evaluated: in our experience, hysteroscopy and biopsy are the more reliable means of obtaining a diagnosis. With regard to the screening of symptom-free subjects, in our small study with a limited period of follow up. we could not find any case of endometrial cancer using SHG. Larger studies with a longer period of follow up are required to find out whether screening as agreed upon in a recent international meeting [17] (yearly TVUS from 2-3 years after the start of tamoxifen, with hysteroseopy or SHG if there is endometrial thickening) is cost beneficial.

\section{References}

1. Smith Bindman R. Kerlikewske K, leldstein VA, Subak L., Scheidler J, Segal M, Brand R. (irady 1): Indwaginal ultraseund to exclude endometrial cancer and wher endometrial ab. normalities. JAMA 2811.15111 .1517 , 1948 
2. Fisher $\mathrm{B}$, Constantino IP, Redmond $\mathrm{Cl}$, et al.: Endometrial cancer in tamoxifen-treated breast cancer patients: lindings from the National Surgical Adjuvant Breast and Bowel Project (NSABP) B-14. J Natl Cancer Inst 86:527-537, 1994

3. Suh-Bergmann EJ, Goodman A: Surveillance for endometrial cancer in women receiving tamoxifen. Ann Intern Med 131:127-135, 1999

4. Cohen I, Rosen DID, Shapira I, et al.: Endometrial changes with tamoxifen: comparison between treated and non-treated asymptomatic, postmenopausal brcast cancer patients. Gynecol Oncol 52:185-190, 1994

5. Lahti E, et al.: Endometrial changes in postmenopausal breast cancer patients receiving tamoxifen. Obstet Gynecol 81:660-664, 1993

6. Cecchini S, Ciatto S, Bonardi R, et al.: Screening by ultrasonography for cndometrial carcinoma in postmenopausal breast cancer patients under adjuvant tamoxifen. Gynecol Oncol 60:409-411, 1996

7. Bertelli G, Venturini M, Del Mastro L, et al.: Tamoxifen and the endometrium: findings of pelvic ultrasound examination and endometrial biopsy in asymptomatic breast cancer patients. Breast Cancer Res Treat 47:41-46, 1998

8. Love CDB, Muir BB, Scrimgeour JB, Leonard RCF, Dillon P, Dixon IM: Investigation of endometrial abnormalities in asymptomatic women treated with tamoxifen and an evaluation of the role of endometrial screening. J Clin Oncol 17:2050-2054, 1999

9. Barakat RR: Screening for endometrial cancer in the patient receiving tamoxifen for breast cancer. J Clin Oncol 17:1967-1968, 1999

10. Tepper R, Beyth Y, Altaras MM, Zalel Y, Shapira J, Cordoba M, Cohen I: Value of sonohysterography in asymptomatic postmenopausal tamoxifen-treated patients. Gynecol Oncol 64:386-391, 199711. Achiron R, Lipitz S, Sivan E, Goldenberg M, Mashiach S: Sonohysterography for the ultrasonographic evaluation of tamoxifen-associated cystic thickened endometrium. J Ultrasound Med 14:685-688, 1995

11. Timmerman D, Deprest J, Bourne T, Van den Berghe I, Collins WP, Vergote I: A randomized trial on the use of ultrasonography or office hysteroscopy for endometrial assessment in postmenopausal patients with breast cancer who were treated with tamoxifen. Am J Obstet Gynecol 179:62-70, 1998

12. MCGonigle KF, Shaw SL, Vasilev SA, Odom-Maryon T, Roy S, Simpson IF; Abnormalities detected on transvaginal ultrasonography in tamoxifen-treated postmenopausal breast cancer patients may represent endometrial cystic atrophy. Am J Obstet Gynecol 78:1145-1150, 1998

13. Mourits MIE, Van der Zee AGI, Willemse PHB, Ten Hoor KA, Hollema H, De Vries EGE: Discrepancy between ultrasonography and hysteroscopy and histology of endometrium in postmenopausal breast cancer patients using tamoxifen. Gynecol Oncol 73:21-26, 1999

14. Schwartz LB, Snyder I, Horam C, Forges RF, Nachtigall IE, Goldstein SR: The use of transvaginal ultrasound and saline infusion sonohysterography for the evaluation of asymptomatic postmenopausal breast cancer patients on tamoxifen. Ultrasound Obstet Gynecol 11:48-53, 1998

15. Dal Cin P, Timmerman D, Van den Berghe I, Wanschura S, Kazmierczack B, Vergote I, Deprest I, Neven P, Moerman P, Bullerdiek J, Van den Berghe H: Genomic changes in endometrial polyps associated with tamoxifen show no evidence for its action as an external carcinogen. Cancer Res 58:2278-2281, 1998

16. Neven P, Vergote I: Should tamoxifen users be screened for endometrial lesions? Lancet $351: 155-157,1998$ 


\section{Chapter 4}

\section{Toremifene as a substitute for adjuvant tamoxifen in breast cancer patients}

Gianfilippo Bertelli, Paola (Yueirolo. Stelania Vecehio. Catia Angiolini, Marina Bergaglio, I ucia del Mastro, Alessandma Signorini. Mario Valenzano and Marren Venturini

Anticancer Resectroh 20101):20:3659-61 


\section{Summary}

Background. Toremifene is a new antiestrogen, which in non-clinical studies appears less carcinogenic than tamoxifen. Clinical trials of adjuvant toremilene vs. tamoxifen in breast cancer patients are ongoing. This study aimed to evaluate the short-term effects of changing from adjuvant tamoxifen to toremi fene.

Patients and Methods. Twenty postmenopausal breast cancer patients receiving adjuvant tamoxifen, 20 $\mathrm{mg} / \mathrm{day}$, were switched to toremifene $60 \mathrm{mg} / \mathrm{day}$. The effects on the uterus were evaluated prospectively by transvaginal ultrasound; tolerability was assessed clinically.

Results. In 14 palients who had uterine abnormalities (endometrial thickening or polyps) under tamoxilen, no significant changes occurred during a median of 18 months (range 7-24) of toremifene treatment. Out of six patients who had entered the study due to intolerance to tamoxifen, however, 3 tolerated toremifene well.

Conclusion. Toremifene does not modify previous uterine changes induced by tamoxifen. For some patients who do not tolerate tamoxifen, however, switching to toremifene may allow the continuation of adjuvant antiestrogenic therapy:

\section{Introduction}

The non-steroidal antiestrogen tamoxifen is currently used in all stages of breast cancer. As an adjuvant therapy after surgery for primary tumors, it prolongs disease free and overall survival and reduces the risk of contralateral breast cancer [1]. In this setting, the benefits of tamoxifen clearly outweigh its potential toxicity, including an increased risk of endometrial cancer [2]. However, in order to develop alternative adjuvant treatments with a better toxicity profile, a number of new hormonal agents are currently under clinical investigation. Toremifene is a new antiestrogen that has been approved for the treatment of metastatic breast cancer in postmenopausal women, where it has demonstrated comparable efficacy to tamoxifen [3]. Contrary to tamoxifen, toremifene is not carcinogenic in the rat $[4,5]$ and produces few or no DNA adducts in vivo [6]. In 1996, the International Agency for Research on Cancer (IARC) concluded that tamoxifen was a human carcinogen, while toremifene was not classifiable [7]. If ongoing clinical studies prove that toremifene is at least as effective as tamoxifen in the adjuvant setting and that its improved pre-clinical safety translates into a reduced risk of secondary cancers, some patients receiving adjuvant tamoxifen may wish to change to toremifene. A crossover from tamoxifen to toremifene, however, is not included in the design of adjuvant trials comparing tamoxifen with toremifene [8] and in only one of the randomized trials in advanced breast cancer [9].

We have prospectively evaluated a group of breast cancer patients receiving tamoxifen as adjuvant therapy, who were switched to toremifene. Inclusion in the study was limited to patients who had asked to interrupt tamoxifen due to systemic side effects or because of concern for gynecological abnormalities. Such patients would be more likely to be offered a change in their treatment if an alternative to tamoxifen in the adjuvant setting became available.

\section{Patients and Methods}

Asymptomatic, postmenopausal breast cancer patients receiving adjuvant tamoxifen $(20 \mathrm{mg} /$ day $)$, who had tamoxifen- associated gynecological abnormalities, or had persis- 
tent side effects under tamoxifen (with the exception of hot flushes), were eligible for this study. After obtaining informed consent, patients were switched to toremifene $(60 \mathrm{mg} / \mathrm{day})$ for the remaining time of their planned adjuvant hormone therapy, i.e. for up to a total of five years. Gynecological examination and transvaginal ultrasonography were performed before starting treatment with toremifene and repeated every 6 months. Transvaginal ultrasonography was performed with an Aloka SSD 680 system using a 5.0 MHz vaginal probe (Aloka, Tokyo, Japan). Anteroposterior measurements of endometrial thickness were obtained from the long-axis view, recording the maximum width between the lines separating the hyperechogenic endometrium from the myometrium. When necessary during treatment with toremifene, i.e. if vaginal bleeding or other symptoms appeared, hysteroscopy and endometrial biopsy were also performed. Side effects and compliance to treatment were evaluated clinically at three-monthly intervals for the first year, then every six months.

\section{Results}

Twenty patients entered into the study between October 1997 and April 1999. The main characteristics of the patients are summarized in Table 1.

TABLE 1

Patients characteristics.

\begin{tabular}{ll}
\hline No. of patients & 20 \\
\hline Median age: years (range) & $63(45-77)$ \\
Median menopausal age: months (range) & $130(6-360)$ \\
Hormone receptor status: & \\
ER + PgR + & $7(35 \%)$ \\
ER + PgR - & $5(25 \%)$ \\
ER - PgR + & $1(5 \%)$ \\
ER - PgR - & $5(25 \%)$ \\
Unknown & $2(10 \%)$ \\
Duration of Tamoxifen: & \\
Mean: months (range) & $26(4-105)$ \\
Previous adjuvant chemotherapy: & \\
No & $12(60 \%)$ \\
Yes & $8(40 \%)$ \\
\hline
\end{tabular}

Based on the eligibility criteria, the effects of changing to toremifene were analyzed in 2 groups of patients:

Patients with gynaecological abnromalities under tamoxifen $(n=14)$. Patients in this group had abnormal ( $>5 \mathrm{~mm}$ ) endometrial thickness at TVUS under tamoxifen; in 5 patients the presence of a polyp was also diagnosed by sonohysterography or hysteroscopy. In four patients, the effects of changing to toremifene were not evaluable: two patients stopped the treatment before re-evaluation because of side effects (gastric pain, nausea and vomiting), one patient died for non-cancer causes and another was lost to follow-up. In the remaining 10 evaluable patients, after a median duration of toremifene treatment of 18 months (range 7-24), endometrial thickness remained abnormal in all cases. One patient had vaginal bleeding and underwent hysterectomy, which 
showed benign histology (glandulocystic hypertrophy). In two patients polyps were excised and examined: one was a fibrotic polyp, the other showed glandulocystic hypertrophy. The remaining three polyps did not change and were followed up by TVUS.

Patients with intolerance of tamoxifen $(n=6)$ : Three patients in this group had complete resolution of side effects previously reported with tamoxifen (pruritus, skin rash and gastric pain respectively) after switching to toremifene. The other three patients had no significant change in toxicity (dizziness, dysuria and fluid retention respectively) and stopped the treatment. TVUS findings before and after toremifene in this group showed no significant changes.

None of the patients in this study has had breast cancer relapse to date.

\section{Discussion}

Although the actual incidence of secondary cancers with tamoxifen is relatively low, of the order of 2 out of 1000 patients/year for endometrial cancer [10], this potential is often the cause of great concern for patients receiving long-term treatment after surgery for primary breast cancer. Screening with ultrasound in this higher-than-average risk group has been advocated, but the lack of correlation between ultrasound findings and endometrial pathology in patients treated with tamoxifen limits its usefulness [11,12]. Nonetheless, the diagnosis of abnormalities such as endometrial thickening and polyps contributes to the patients' worries, even when not accompanied by clinical symptoms. The desire to improve the safety of adjuvant hormone therapy of breast cancer has provoked a search for agents with less toxicity to the uterus. Toremifene is a new antiestrogen with a good safety profile and clinically equivalent efficacy compared to tamoxifen in advanced breast cancer [13]. In the adjuvant setting, toremifene has not yet been adequately studied; an improved non-clinical safety profile holds promise for a reduced risk of endometrial cancer [6], although this concept has recently been challenged by the finding of similar effects of tamoxifen and toremifene on the growth of endometrial cancer in athymic mice [14].

In humans, toremifene appears to have estrogen- like effects on the uterus very similar to those already known for tamoxifen. A prospective, randomized clinical study by Tomas et al has compared the effects of tamoxifen and toremifene on the uterus in postmenopausal breast cancer patients [15]. The authors reported comparable estrogenic effects of the two drugs on the endometrium and on the vaginal epithelium. There were no significant differences between tamoxifen and toremifene in endometrial thickness, occurrence of polyps and changes in Pap smear.

The endometrial effects of crossing over from tamoxifen to toremifene had not been described previously. Although long-term effects still need to be further investigated, our study suggested that no significant change in the TVUS-detected, asymptomatic, endometrial abnormalities frequently associated with tamoxifen use can be expected when switching to toremifene. On the other hand, when the crossover to toremifene was justified by previous toxicity with tamoxifen, a possible benefit was suggested by our study. Although the number of subjects in this group was small, three out of six patients with persistent side effects with tamoxifen showed excellent tolerance to toremifene. In such cases, switching from tamoxifen to toremifene might offer a possibility of completing the planned adjuvant antiestrogenic treatment. In conclusion, although long-term 
consequences still need to be investigated, available data suggest that the effects of toremifene on the human uterus are very similar to those of tamoxifen. When used upfront, toremifene induces the same uterine changes (endometrial thickening, development of polyps) that are commonly associated with tamoxifen use in postmenopausal patients [15]. Our study showed that a crossover from tamoxifen to toremifene did not modify previous tamoxifen-induced uterine abnormalities. However, there is no clear correlation between such changes, which can be found in the majority of women receiving tamoxifen and are usually asymptomatic $[12,16]$ and the development of endometrial cancer in a much smaller fraction of patients. Only with sufficient follow-up of ongoing phase III adjuvant trials, the role of toremifene and its possible advantages over tamoxifen will be adequately defined.

\section{References}

1. Early Breast Cancer Trialists' Collaborative Group. Tamoxifen for early breast cancer: an overview of the randomised trials. Lancet 557: 1451-1467, 1998.

2. Fisher B, Costantino JP, Redmond CK, Fisher ER, Wickerham DL and Cronin WM, et al: Endometrial cancer in tamoxifen-treated breast cancer patients: findings from the National Surgical Adjuvant Breast and Bowel Project (NSABP) B-14. J Natl Cancer Inst 86: 527 $537,1994$.

3. Buzdar AU and Hortobagy GN: Tamoxifen and toremifene in breast cancer: comparison of safety and efficacy. J Clin Oncol 16: 348-353, 1998.

4. Hard GC, latropoulos $\mathrm{Mj}$ and Jordan $\mathrm{K}$ et al: Major difference in the hepatocarcinogenicity and DNA adduct forming ability between toremifene and tamoxifen in female $\mathrm{Crl}: \mathrm{CD}(\mathrm{BR})$ rats. Cancer Res 55:4534-4541,1993.

5. Karlsson S, Hirsimaki Y and Mantyla L, et al: A two-year dietary carcinogenicity study of the antiestrogen toremifene in Sprague-Dawley rats. Drug Chem Toxicol 19:245-266, 1996.

6. Williams GM and Jeffrey AM: Safety assessment of tamoxifen and toremifene. Oncology (Hunting.) 11(5 suppl 4): 41-47, 1997.

7. International Agency for Research on Cancer. Tamoxifen. In: IARC Monographs on the Evaluation of Carcinogenic Risks to Humans.: Some Pharmaceutical Drugs, vol. 66, pp 253387. IARC Press, Lyon, France 1996.

8. Holli K: Adjuvant trials of toremifene vs tamoxifen: the European experience. Oncology (Hunting!) 72(5 suppl 5): 23-27, 1998.

9. Stenbygaard LE, Herrsted I, Thomsen JF, Svendsen KR, Engelholm SAA and Dombernowsky P: Toremifene and tamoxifen in advanced breast cancer a double-blind cross-over trial. Breast Cancer Res Treat 25: 57-63,1993.

10. Jordan VC and Assikis VJ: Endometrial carcinoma and tamoxifen: clearing up a controversy. Clin Cancer Res 1: 467-472,1995.

11. Barakat RR: Screening for endometrial cancer in the patient receiving tamoxifen for breast cancer. J Clin Oncol 17: 1967-1968, 1999.

12. Love $\mathrm{CDB}$, Muir BB and Scrimgeour JB, et al: Investigation of endometrial abnormalities in asymptomatic women treated with tamoxifen and an evaluation of the role of endometrial screening. J Clin Oncol 17: 2050-2054, 1999. 
13. Pyrhonen S, Ellmen J, Vuorinen J, Gershanovich M, Tominaga T, Kaufmann $M$ and Hayes DF: Meta-analysis of trials comparing toremifene with tamoxifen and factors predicting outcome of antiestrogen therapy in postmenopausal women with breast cancer. Breast Cancer Res Treat 56: 133-143,1999.

14. O'Regan RM, Cisneros A and England GM, et al: Elfects of the antiestrogens tamoxifen, toremifene and ICI 182,780 on endometrial cancer growth. J Natl Cancer Inst 90: 15521558, 1998.

15. Tomas E, Kauppila A, Blanco G, Apaja-Sarkkinen M and Laati-kainen T: Comparison between the effects of tamoxifen and toremifene on the uterus in postmenopausal breast cancer patients. Gynecol Oncol 59: 261-266,1995.

16. Bertelli G, Venturini $M$ and Del Mastro $L$ et al: Tamoxifen and the endometrium: findings of pelvic ultrasound examination and endometrial biopsy in asymptomatic breast cancer patients. Breast Cancer Res Treat 47: 41-46, 1998. 


\section{Chapter 5}

\section{Intramuscular depot medroxyprogesterone versus oral megestrol for the control of postmenopausal hot flashes in breast cancer patients: a randomized study}

G. Bertelli, M. Venturini, L. Del Mastro, M. Bergaglio, P. Sismondi, N. Biglia, S. Venturini, G. Porcile, P. Pronzato, M. Costantini and R. Rosso

Annals of Onologe: 13: $8 \times 3-848,200$ ? 


\begin{abstract}
Summary
Background. Hot llashes are frequent in postmenopausal breast cancer patients, especially when treated with tamoxifen. Estrogen replacement therapy is the most effective treatment for hot hashes, but its use is controversial in breast cancer survivors. Progestins may offer a good alternative for the control of hot flashes in this setting; in particular, oral megestrol acetate has been proven effective in a randomized, placebo-controlled clinical trial. With the aim of further improving these results, we have designed a randomized study comparing oral megestrol acetate with depot intramuscular (i.m.) medroxyprogesterone acetate (MPA) for the control of hot flashes in postmenopausal patients with a history of breast cancer.

Patients and Methods. Seventy-one postmenopausal paticnts were randomized to receive an i.m. injection of depot MPA $500 \mathrm{mg}$ on days 1,14 and 28, or oral megestrol acetate $40 \mathrm{mg}$ daily for 6 weeks. Patients recorded daily the number and severity of their hot flashes; response was delined as a $\geq 50 \%$ decrease in the number and severity of hot flashes.

Results. At week 6 , hot flashes were reduced by $86 \%$ on avernge in the whole group of patients, without significant differences between the two progestins. Response was obtained in 75 and $67 \%$ of patients receiving MPA or megestrol, respectively $(p=0.5)$. Responders were followed to assess maintenance of response (without further treatment), which was significantly better with i.m. MPA: in this group, $89 \%$ of responders still showed a benetit at week 24 , compared with $45 \%$ in the megestrol group $(p=0.03)$.

Conclusions. Our study shows that a short cycle of i.m. depot MPA injections provides significant and longlasting relief from postmenopausal hot tlashes in patients with a history of breast cancer, offering an alternative to estrogen replacement therapy or prolonged administration of oral megestrol.
\end{abstract}

\title{
Introduction
}

Hot flashes are among the most frequently reported sequelae in breast cancer survivors; their incidence ranges from 55 to $65 \%$ in recent surveys $[1,2]$. In one study the overall prevalence of hot flashes in breast cancer patients was $65 \% ; 44 \%$ of patients reported their hot flashes as 'severely bothersome', and 63\% expressed an interest in learning about management strategies [3]. Treatment with the antiestrogen tamoxifen was associated with increased severity and higher prevalence $(78 \%)$ of hot flashes.

Estrogen replacement therapy is considered the most effective treatment for postmenopausal hot flashes; however, the prescription of estrogens in breast cancer survivors is avoided by many clinicians because of a theoretical increased risk of relapse associated with their use.

The administration of progestational agents is a possible alternative to estrogens, offering good control of hot flashes in most patients. In a placebo-controlled, double blind randomized study, megestrol acetate reduced hot flashes by $85 \%$ in a group of breast and prostate cancer patients when administered at a dose of $40 \mathrm{mg} /$ day orally for 4 weeks [4]. The study did not evaluate the duration of response obtained with megestrol acetate; however, the results of a follow-up investigation showed that some patients chose to keep on taking this medication for up to 3 years [5]. This may be interpreted as indirect evidence that long-term treatment with megestrol is necessary to maintain the initial benefit.

Another progestin, medroxyprogesterone acetate (MPA), has been in use for many years for the treatment of hot flashes in healthy postmenopausal women [6]. Intramuscular administration of a depot formulation of MPA has been shown to be effective against hot flashes in randomized, placebo-controlled studies [7, 8]. An interesting characteristic of depot MPA is its long duration of action, due to the fact that progestin is released 
slowly from the muscle after the injection [9]. In our experience, some breast cancer patients benefit from a dramatic and prolonged reduction of their hot flashes after a short initial cycle of depot MPA injections. Based on these encouraging observations, we designed a randomized trial to compare this treatment modality with oral megestrol acetate in a group of breast cancer survivors.

\section{Patients and Methods}

Eligible patients were postmenopausal women with a history of breast cancer and no evidence of relapse, who had been suffering with bothersome hot flashes for at least 1 month before entry into the study. Hot flashes were defined as 'bothersome' if they occurred at least seven times per week and were sufficiently severe that the patient asked for therapeutic intervention. Postmenopausal status was defined as an absence of regular menses for $\geq 6$ months in patients with an intact uterus; patients with previous hysterectomy but no bilateral oophorectomy were considered postmenopausal if plasma follicle-stimulating hormone, leutinizing hormone and estradiol were in the postmenopausal range. No concurrent or planned treatment with estrogens, androgens, progestins, corticosteroids, clonidine, veralipride or ciclophenile was allowed; patients who had received adjuvant chemotherapy had to have concluded treatment for $\geq 2$ months. Concurrent adjuvant tamoxifen $20 \mathrm{mg} /$ day was allowed if started at least 1 month before entry in to the study and if the planned residual duration of treatment was at least 6 months. Written informed consent was obtained from all patients.

Patients were randomized to one of two groups: group 1 received intramuscular (i.m.) depot MPA (500 mg i.m. on days 1, 14 and 28); group 2 received oral megestrol acetate (40 mg p.o. once daily from day 1 to day 42 ). Treatment allocation was not double blinded because patients in group 2 would have required the administration of an i.m. placebo. This was judged to be impractical.

The frequency and severity of hot flashes in the participating patients were monitored through a self-compiled diary, which was started 7 days before the beginning of treatment (to provide baseline data) and continued for 6 weeks thereafter. The diary had columns for each day of the week and rows labeled 'mild', 'moderate', 'severe' and 'very severe'. Patients were asked to record every day the number of hot flashes that they had suffered and their severity, assigning them to one of the four grades detailed above. Choice of grade was subjective. No definition of mild, severe or very severe was given to the patients, who were only asked to be consistent over the duration of the study. At the end of each week, patients were also asked to record in their diary all postmenopausal symptoms, other than hot flashes, or side effects of therapy encountered during the last 7 days. Patients could choose items from a list that included insomnia, fatigue, mood instability, vaginal discharge, dizziness, appetite increase, appetite decrease, dry mouth and fluid retention.

Three main efficacy parameters were evaluated at the end of the sixth week from the start of treatment (i.e. after 6 weeks of daily oral treatment with megestrol acetate or 2 weeks after the last of three i.m. injections of depot MPA), using the average values recorded for that week in comparison to baseline values calculated during the initial week with no treatment. For each group, changes in average number of hot flashes per day and average daily hot-flash score were analyzed using values recorded in the first 
week (with no treatment) as a baseline. The average daily hot-flash score for each patient was calculated by adding the number of mild hot flashes plus twice the number of moderate hot flashes plus three times the number of severe hot flashes plus four times the number of very severe hot flashes in a week, and then dividing the sum by seven. The third parameter was the proportion of patients who obtained a $\geq 50 \%$ reduction in the frequency of hot flashes and hot-flash score, as compared with baseline values. This value was arbitrarily chosen as a threshold for a clinically significant result, and was used to define 'responding' patients. All patients who achieved a $<50 \%$ reduction, or who did not complete the treatment for any reason, including side effects or refusal, were defined as treatment failures and withdrawn from the study. Off-study patients, who returned for their normal clinical follow-up, were only asked to report late side effects of treatment such as withdrawal bleeding. Responding patients were asked to continue their diaries for up to a total of 24 weeks from the start of treatment. No maintenance treatment was given in responding patients, who were visited at 2-monthly intervals. When hot flash frequency or score returned to $>50 \%$ of baseline values, the patient was considered to have lost the initial response and was withdrawn from the study.

Statistical analysis. The primary end point of the study was the proportion of responding patients 6 weeks after the planned start of treatment ( 7 weeks after randomization). A patient was classified as a responder if she achieved $a \geq 50 \%$ reduction in the frequency of hot flashes and hot-flash score. Sample size was calculated assuming a proportion of responders in group 2 (receiving megestrol acetate) of $70 \%$, and that the treatment with MPA was associated with a $25 \%$ absolute increase in the proportion of responders (from 70 to $95 \%$ ). For an $80 \%$ power and a two-sided $5 \%$ significance, 90 subjects were planned to detect such a difference. The study was stopped after 71 patients had been randomized over a period of 2 years because of difficulties encountered during patient enrollment. The proportion of responders was compared by means of the chi-square test [10]. Confidence limits for proportions were estimated according to Fleiss [10].

\section{Results}

From June 1996 to June 1998, a total of 71 breast cancer patients were randomized to group 1 (i.m. MPA; $n=37$ ) or group 2 (oral megestrol acetate; $n=34$ ). As can be seen from Table 1, which summarizes the main characteristics of patients in the two groups, the majority of patients in the study were receiving adjuvant tamoxifen $(n=52,73.2 \%)$. The median duration of tamoxifen treatment was 10 months (range 1-43); although eligibility criteria allowed patients to be entered after 1 month of tamoxifen treatment. most patients ( 34 of 52,65.4\%) had been using tamoxifen for at least 6 months.

After randomization, five patients in each group refused to start the assigned treatment and withdrew from the study. Two more patients, both in group 1, were found to be ineligible after randomization (one for medical contraindications to progestin treatment, one not postmenopausal). Six patients did not provide complete diary recordings during treatment (five patients who dropped out before completion for side effects and one who was lost to follow-up). 
TABLE 1

Patient characteristics.

\begin{tabular}{|c|c|c|}
\hline & Medroxyprogesterone & Megestrol \\
\hline \multicolumn{3}{|l|}{ Age (years): } \\
\hline Median (range) & $51(40-67)$ & $53(42-72)$ \\
\hline \multicolumn{3}{|l|}{ Months since last period: } \\
\hline Median (range) & $21(1-195)$ & $24(6-199)$ \\
\hline \multicolumn{3}{|l|}{ Previous adjuvant chemotherapy: } \\
\hline No. of patients $(\%)$ & $22(59.4)$ & $22(64.7)$ \\
\hline \multicolumn{3}{|l|}{ Chemotherapy induced menopause: } \\
\hline No. of patients $(\%)$ & $13(35.1)$ & $11(32.3)$ \\
\hline \multicolumn{3}{|l|}{ Months from end of chemotherapy: } \\
\hline Median (range) & $11(4-50)$ & $15(2-26)$ \\
\hline \multicolumn{3}{|l|}{ Tamoxifen use: } \\
\hline No. of patients $(\%)$ & $27(73.0)$ & $25(73.5)$ \\
\hline \multicolumn{3}{|l|}{ Months of Tamoxifen use: } \\
\hline Median (range) & $10(2-43)$ & $11(1-39)$ \\
\hline \multicolumn{3}{|c|}{$\begin{array}{l}\text { Patients on Tamoxifen who also had previous } \\
\text { chemotherapy: }\end{array}$} \\
\hline No. of patients (\% of Tamoxifen users) & I $5(48.4)$ & $16(51.6)$ \\
\hline \multicolumn{3}{|l|}{ Months with hot flushes: } \\
\hline Median (range) & $11.5(1-189)$ & $10.5(1-199)$ \\
\hline \multicolumn{3}{|l|}{ Months since breast cancer diagnosis: } \\
\hline Median (range) & $14(2-220)$ & $17(3-137)$ \\
\hline \multicolumn{3}{|l|}{ Hot flushes number at baseline: } \\
\hline Daily average (mean $\pm \mathrm{SD})$ & $8.8 \pm 4.1$ & $9.2 \pm 6.3$ \\
\hline Range & $2-19$ & $1-30$ \\
\hline \multicolumn{3}{|l|}{ Hot flushes score at baseline: } \\
\hline Daily average (mean $\pm S D$ ) & $19.3 \pm 10.6$ & $20.8 \pm 16.7$ \\
\hline Range & $4-44$ & $2-71$ \\
\hline
\end{tabular}

Reduction of hot flashes. Figure 1 shows changes in the number of daily hot flashes and the daily hot-flash score calculated from diary recordings during the baseline week and the following 24 weeks. The curves are based on all available diary recordings, which were provided by 65 patients $(91 \%)$ at baseline and $53(75 \%)$ at week 6 . The mean number of hot flashes and hot-flash score at baseline did not differ significantly between the two groups. At week 6, the mean number of hot flashes per day was $1.21[95 \%$ confidence interval (CI) $0.65-1.77$ ] in group 1 and 1.42 (95\% CI $0.67-1.17$ ) in group 2; the mean scores were 2.08 (range 1.04-3.12) and 2.34 (range 0.99-3.7), respectively. Differences between the two groups at week 6 were not statistically significant. To assess the relative reduction in the number of hot flashes and the hot-flash score between baseline and week 6 , we considered the 53 patients who provided complete diary recordings at baseline and at week 6 . Overall, the average daily number of hot flashes was reduced by $87.5 \pm 16.7 \%$ (range $28.6-100$ ) as compared with baseline values, and the average daily hot-flash score was reduced by $89.6 \pm 17.1 \%$ (range $6.9-100$ ). The differences between the two groups were not significant. Good control of hot flashes by both treatments is also apparent in the reduction of the frequency of related symptoms, such as insomnia, mood instability and fatigue, as compared with baseline values ( $\mathrm{Ta}$ ble 2). 
FIGURE 1

Changes in daily number of hot flashes (A) and hot-flash score (B) during treatment (first 6 weeks) and up to 24 weeks after randomization in the two treatment groups. The number of patients providing valid diary recordings for each time period is shown under the curves. MPA, medroxyprogesterone acetate.

A average daily score

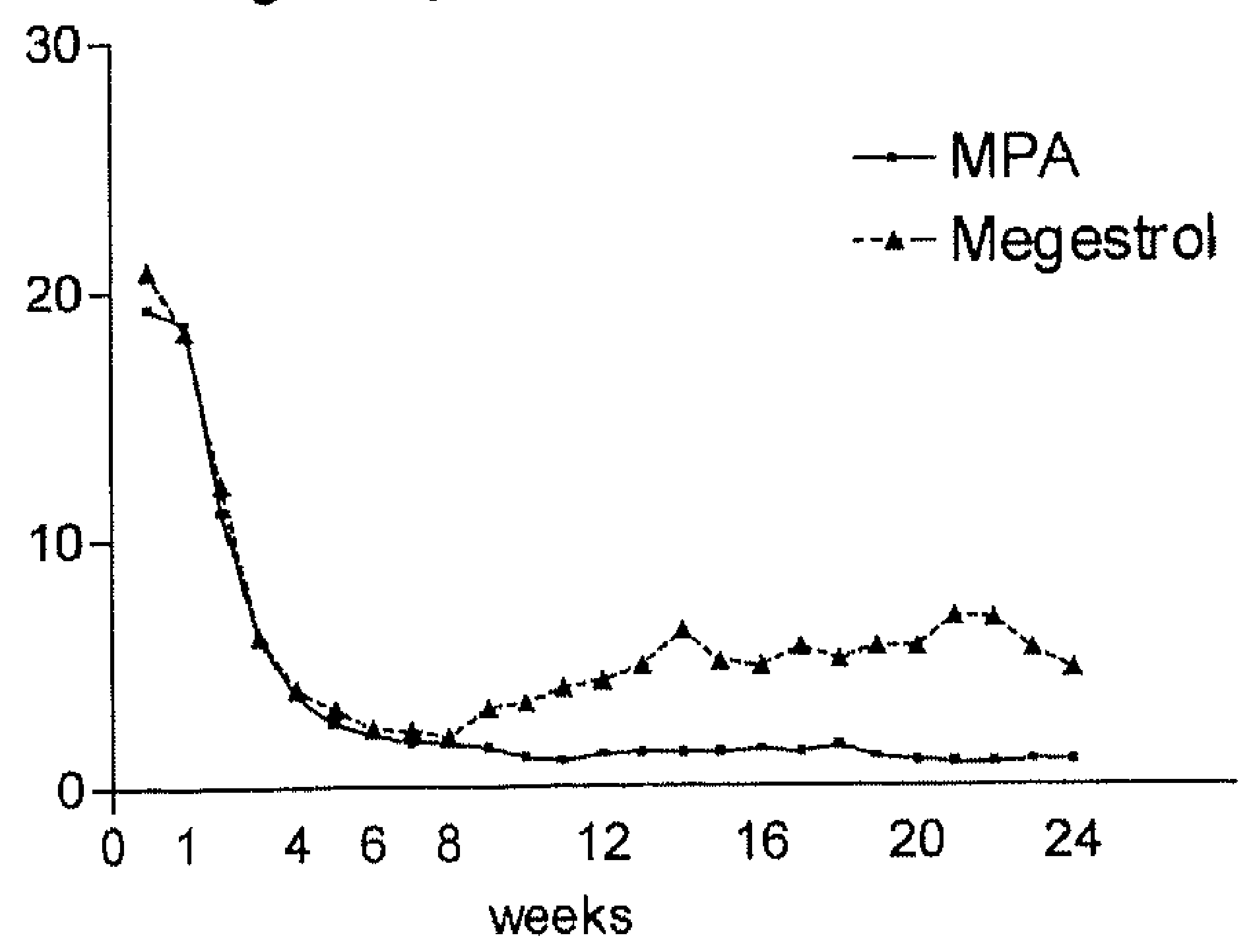

MPA (n=37)

Megestrol $(n=34)$

\begin{tabular}{lllllllll}
34 & 29 & 29 & 29 & 24 & 22 & 23 & 21 & 17 \\
31 & 29 & 27 & 24 & 18 & 18 & 14 & 12 & 10 \\
\multicolumn{4}{c}{ patients at risk }
\end{tabular}

B average daily number

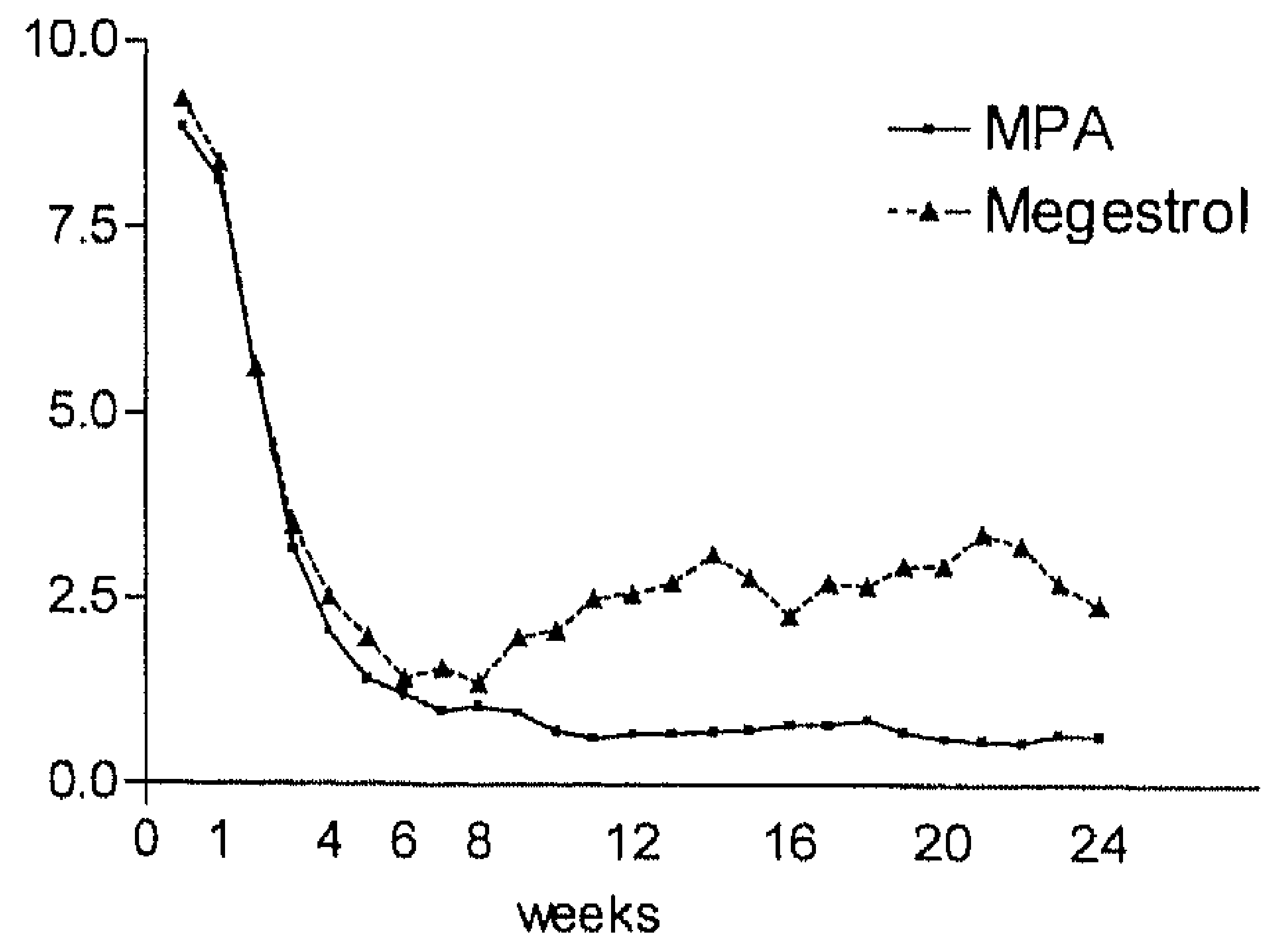

MPA $(n=37)$

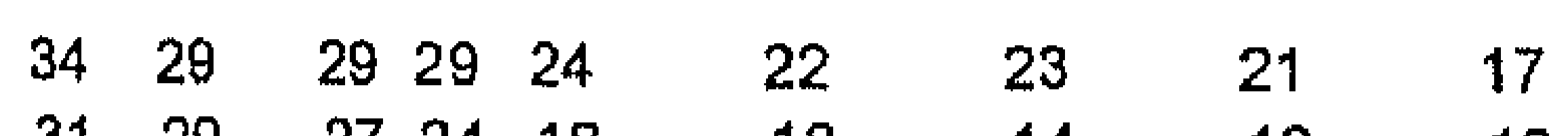

Megestrol $(n=34)$
$\begin{array}{lllllll}31 & 29 & 27 & 24 & 18 & 18 & 14\end{array}$
patients at risk 
TABLE 2

Frequency of other menopausal symptoms.

\begin{tabular}{lll}
\hline & No. of patients $(\%)$ & \\
\hline & $\begin{array}{l}\text { Medroxyprogesterone } \\
\text { (group 1: } \mathrm{n}=37 \text { ) }\end{array}$ & $\begin{array}{l}\text { Megestrol } \\
\text { (group 2: } \mathrm{n}=34)\end{array}$ \\
$\begin{array}{l}\text { At baseline: } \\
\text { Insomnia }\end{array}$ & $20(54.1)$ & $18(52.9)$ \\
Mood instability & $17(45.9)$ & $18(52.9)$ \\
$\quad$ Fatigue & $18(48.6)$ & $15(44.1)$ \\
End of treatment: & & \\
Insomnia & $5(13.5)$ & $5(14.7)$ \\
Mood instability & $4(10.8)$ & $10(29.4)$ \\
Fatigue & $8(21.6)$ & $9(26.4)$ \\
\hline
\end{tabular}

Response rate. Response rate at the end of week 6 was evaluated according to an intention-to-treat criterion on the whole group of 71 randomized patients. Overall, 50 of 71 patients $(70.4 \% ; 95 \%$ CI $58-81 \%)$ achieved a response as previously defined, i.e. $\geq$ $50 \%$ reduction in hot flashes frequency and hot-flash score; this was often a complete response (total disappearance of hot flashes). Response distribution in the two treatment groups was as follows: 28 of $37(75.7 \% ; 95 \%$ CI $59 \%$ to $88 \%)$ in group 1, with nine of $37(24.3 \%)$ treatment failures (seven never started for ineligibility or refusal, one was lost to follow up and one had a $<50 \%$ reduction) and 22 of $34(64.7 \% ; 95 \% \mathrm{Cl} 46 \%$ to $80 \%$ ) in group 2 , with 12 of $34(24.3 \%$ ) treatment failures (five never started for refusal, five stopped early because of side effects, and two had a $<50 \%$ reduction). Responders in group 2 include one patient who had discontinued megestrol acetate before completion of treatment due to a skin rash, but provided complete recordings in her diary showing $>50 \%$ reduction of hot flashes frequency and hot-flash score at the end of week 6 . No significant difference between the proportion of responders between the two arms was observed ( $P=0.567)$. Table 3 shows the distribution of responses in the two groups according to the number of hot flashes at baseline. The number of hot flashes at baseline was not significantly associated with the proportion of responses $(P=0.188)$.

TABLE 3

Reduction of hot flashes after 6 weeks, according to the number or hot flashes at baseline, in the two groups.

\begin{tabular}{lll} 
& \multicolumn{2}{c}{$\begin{array}{c}\text { No. }(\%) \text { of patients with } \\
\geq 50 \% \text { reduction in hot flushes }\end{array}$} \\
\hline $\begin{array}{l}\text { Average no. of daily hot flushes } \\
\text { at baseline": }\end{array}$ & Medroxyprogesterone & Megestrol \\
$1-3$ & (group 1) & (group 2) \\
$4-9$ & $3 / 4(75.0)$ & $1 / 5(20.0)$ \\
$\geq 10$ & $12 / 17(70.6)$ & $11 / 14(78.6)$ \\
& $13 / 13(100)$ & $10 / 12(83.3)$ \\
"Data not available in six patients. & & \\
\hline
\end{tabular}

Response duration. Maintenance of response in the group of 50 initial responders was assessed at 2-monthly follow-up visits, for 6 months after randomization. However, due to a reduction in the number of patients filling in their diaries during this part of the study, complete diary data were not available for $25(50 \%)$ patients. In these patients, the duration of benefit was assessed by asking, at each visit, whether they thought the 
initial treatment benefit was still present. When this was not the case, the patient was considered as having lost her response and was withdrawn from the study. We had found during the first part of the study that the correlation between the overall judgement of benefit from treatment, given by patients and their diary recordings was good. Taking into account these limits, a difference between the maintenance of response with MPA and megestrol was observed. Out of 28 responding patients in the MPA group, 25 $(89.3 \%)$ were still responding at 6 months from randomization. In the megestrol group, only 10 of 22 initial responders $(45.4 \%)$ were still in response after 6 months. In this latter group, moreover, it was found that four patients had continued to use megestrol tablets, up to a maximum of 3 months in one patient and 6 months in three patients. None of the patients randomized to MPA received additional injections after the three initial ones. The difference in response maintenance at 6 months after randomization is significantly in favor of MPA, even without removing the four protocol violators from the megestrol group $(P=0.03)$.

Tolerability. Although the treatment in both arms was generally well tolerated, more patients in the megestrol group experienced adverse effects, which in six women $(16.6 \%)$ led to early discontinuation of therapy (no patient in the MPA group interrupted treatment). Reasons for interruption were skin rashes in two patients (5.9\%), dyspnoea in two patients $(5.9 \%)$, gastric pain in one patient $(2.9 \%)$ and increased arterial blood pressure in one patient $(2.9 \%)$. Table 4 shows the other side effects reported during treatment. Withdrawal bleeding after the end of treatment was experienced by two patients $(5.4 \%)$ in the MPA group and seven (18.9\%) in the megestrol group.

TABLE 4

Reported adverse effects during treatment.

\begin{tabular}{lll} 
& No. of patients (\%) & \\
\hline Fluid retention & $3(8.1)$ & $6(17.6)$ \\
Dizziness & $5(13.5)$ & $8(23.5)$ \\
Vaginal dryness & $10(27.0)$ & $9(26.5)$ \\
Dry mouth & $12(32.4)$ & $16(47.0)$ \\
\hline
\end{tabular}

\section{Discussion}

The management of hot flashes in postmenopausal women with a history of breast cancer is an important part of clinical practice in oncology, especially considering the increasing number of patients who receive adjuvant treatments and have a long life expectancy. Hot flashes may be associated with premature menopause induced by chemotherapy; moreover, they are the most common side effect of treatment with tamoxifen. Since estrogen replacement therapy is usually avoided in women with a history of breast cancer, patients should be informed of the possible alternatives. Among nonhormonal remedies, clonidine [11], vitamin E [12] and soy phytoestrogens [13] have shown either limited or no benefit in placebo-controlled trials. Until recently, the only treatment with proven benefit in this setting was the progestational agent megestrol acetate, which at the dose of $40 \mathrm{mg} /$ day p.o. was superior to placebo in a randomized, double-blind study [4]. Some of the women obtaining control of their hot flashes with megestrol may choose to continue the treatment at a low dose for years, in order to 
maintain the initial benefit [5]. Although there are no definitive data on the safety of progestin use in women with a history of breast cancer, concerns about its prolonged use may limit the prescription of megestrol acetate for hot flashes.

Another progestational agent, MPA, has been in use for some years in healthy postmenopausal women with hot flashes; oral and parenteral formulations have been evaluated in controlled clinical trials [6-8]. To our knowledge, our study is the first were depot i.m. MPA was used for hot flashes in breast cancer survivors, many of whom were concurrently receiving the antiestrogen tamoxifen. Dosages and schedule of MPA in this study were based on previous empirical observations of long-term relief of hot flashes after a short cycle of i.m. injections in some of our patients. Although no definitive conclusions can be drawn from the comparison with oral megestrol, due to the small number of patients recruited and the difficulty in obtaining valid diary recordings from all subjects during treatment and follow-up, our results suggest that depot MPA can be expected to abrogate or substantially reduce hot flashes in most breast cancer patients. Moreover, nearly $90 \%$ of patients responding to MPA in our study maintained the initial benefits of treatment for 6 months without further treatment. Thus, when considering progestin therapy for the control of hot flashes in breast cancer survivors, depot MPA can be regarded as a reasonable treatment option. Occasionally a patient does not respond to progestins; in this case, other treatments should be offered with the aim of obtaining satisfactory control of the symptom. Encouraging results have recently been reported with low-dose antidepressants, such as paroxetine [14] and venlafaxine [15], suggesting that they may play an important role in this field.

\section{References}

1. Ganz PA, Rowland JH, Desmond K et al. Life after breast cancer: understanding women's health-related quality of life and sexual functioning. J Clin Oncol 1998; 16: 501-514.

2. Couzi RJ, Helzlsouer KJ, Fetting JH. Prevalence of menopausal symptoms among women with a history of breast cancer and attitudes toward estrogen replacement therapy. J Clin Oncol 1995; 13: 2737-2744.

3. Carpenter JS, Andrykowski MA, Cordova $M$ et al. Hot flashes in postmenopausal women treated for breast carcinoma. Cancer 1998; 82: 1682-1691.

4. Loprinzi CL, Michalak JC, Quella SK et al. Megestrol acetate for the prevention of hot flashes. N Engl J Med 1994; 331: 347-352.

5. Quella SK, Loprinzi CL, Sloan JA et al. Long term use of megestrol acetate by cancer survivors for the treatment of hot flashes. Cancer 1998; 82: 1784-1788.

6. Schiff I, Tulchinsky D, Cramer D, Ryan KJ. Oral medroxyprogesterone in the treatment of postmenopausal symptoms. JAMA 1980; 244: 1443-1445.

7. Bullock JL, Massey FM, Gambrell RD. Use of medroxyprogesterone acetate to prevent menopausal symptoms. Obstet Gynecol 1975; 46: 165-168.

8. Morrison JC, Martin DC, Blair RA et al. The use of medroxyprogesterone acetate for relief of climacteric symptoms. Am J Obstet Gynecol 1980; 138: 99-104.

9. Mishell DR. Pharmacokinetics of depot medroxyprogesterone acetate contraception. J Reprod Med 1996; 41: 381-390.

10. Fleiss JL. Statistical methods for rates and proportions. New York, NY: John Wiley 1981. 
11. Goldberg RM, Loprinzi CL, O'Fallon JR et al. Transdermal clonidine for ameliorating tamoxifen-induced hot flashes. J Clin Oncol 1993; 12: 155-158.

12. Barton DL, Loprinzi CL, Quella SK et al. Prospective evaluation of vitamin $E$ for hot flashes in breast cancer survivors. J Clin Oncol 1998; 16: 495-500.

13. Quella SK, Loprinzi CL, Barton DL et al. Evaluation of soy phytoestrogens for the treatment of hot flashes in breast cancer survivors: a North Central Cancer Treatment Group trial. J Clin Oncol 2000; 18: 1068-1074.

14. Stearns V, Issacs $\mathrm{C}$, Rowland J et al. A pilot trial assessing the efficacy of paroxetine hydrocloride (Paxil) in controlling hot flashes in breast cancer survivors. Ann Oncol 2000; 11: 17-22.

15. Loprinzi CL, Kugler JW, Sloan $\mathbf{J}$ et al. Venlafaxine in management of hot flashes in survivors of breast cancer: a randomised controlled trial. Lancet 2000; 356: 2059-2063. 


\title{
Chapter 6
}

\author{
A randomized trial of exemestane after \\ two to three years of tamoxifen therapy \\ in postmenopausal women with primary \\ breast cancer
}

Charles Coombes, Emma Hall, Loma J. Cibson, Rohert Pariclaens, Jacek Jassem. Thierry Delozier, Stephen F. Jones, Isabel Alvarez, Cianfilippo Bertelli, Olaf Ortmann, Alan S. Coates, Emilio Bajetta, David Dodwell. Robert E. Coleman, Lessley J.

Fatlow field, Elizabeth Mickiewicz, Jorn Andersen. Per E. Lonning, Giorgio Coceoni, Alan Stewart, Nick Stuart, Claire F. Snowdon, Marina Carpentieri, Giorgio Massimini. Judith M. Bliss, for the Intergroup Exemestane Study

New Linglund Journal of Medicine 2014:3501:1081-92. 


\section{Summary}

Background. Tamoxifen, taken for live years, is the standard adjuvant treatment for postmenopausal women with primary, estrogen-receptor-positive breast cancer. Despite this treatment, however, some patients have a relapse.

Methods. We conducted a double-blind, randomized trial to test whether, after two to three years of tamoxifen therapy, switching to exemestane was more effective than continuing tamoxifen therapy for the remainder of the five years of treatment. The primary end point was disease-free survival.

Results. Of the 4742 patients enrolled, 2362 were randomly assigned to switch to exemestane, and 2380 to continue to receive tamoxifen. After a median follow-up of 30.6 months, 449 first events (local or metastatic recurrence, contralateral breast cancer, or death) were reported - 183 in the exemestane group and 266 in the recurrence, contralateral bradjusted hazard ratio in the exemestane group as compared with the tamoxifen group tamoxifen group. The unadjusted hazard ratio in the exemestane group as compared 0.68 ( 95 percent confidence interval, 0.56 to $0.82 ; \mathrm{P}<0.001$ by the log-rank test), reprenting a 32 percent reduction in risk and corresponding to an absolute benefit in terms of disease-free survival of 4.7 percent $(95$ percent confidence interval, 2.6 to 6.8 ) at three years after randomization. Overall survival was not significantly different in the two groups, with 93 deaths occurring in the exemestane group and 106 in the tamoxifen group. Severe toxic eflects of exemestane were rare. Contralateral breast cancer occurred in 20 patients in the tamoxifen group and 9 in the exemestane group $(P=0.04)$.

Conclusions. Exemestane therapy after two to three years of tamoxifen therapy significantly improved disease-free survival as compared with the standard live years of tamoxilen treatment.

\section{Introduction}

Breast cancer is estrogen-dependent in many cases, and reducing the estrogen levels by means of ovariectomy can cause regression of established disease [1], especially if the tumor is rich in estrogen receptors [2]. The selective estrogen-receptor modulator tamoxifen blocks the action of estrogen by binding to one of the activating regions of the estrogen receptor $[3,4]$. When given to women with estrogen-receptor-positive breast cancer for five years after surgery, tamoxifen reduces the risk of recurrence by 47 percent and the risk of death by 26 percent [5]. The risk-benefit ratio of using tamoxifen for longer than five years remains unclear $[6,7]$, and trials addressing this question are ongoing. International guidelines recommend that patients should not receive adjuvant tamoxifen therapy for more than five years outside the context of a clinical trial [8]. Altemative endocrine therapy is often effective after disease has relapsed despite tamoxifen treatment, since at that point, estrogen receptors are still present in most patients [9]. Several trials have confirmed the superiority of aromatase inhibitors over progestins in this setting $[10,11]$. Aromatase is an enzyme that catalyzes the conversion of androgens to estrogens. There are two classes of third-generation oral aromatase inhibitors: irreversible steroidal inactivators, exemplified by exemestane $[12,13]$, and reversible nonsteroidal inhibitors, such as anastrozole and letrozole [14].

Exemestane inhibits aromatization in vivo by about 98 percent [15]. It is superior to megestrol acetate with respect to time to progression in advanced breast cancer [14] and has antitumor effects in patients who have no response to third-generation nonsteroidal aromatase inhibitors [16]. Preliminary results show that exemestane is superior to tamoxifen as first-line therapy for metastatic disease [17]. Theoretically, exemestane should not cause endometrial thickening or endometrial cancer, which are occasionally observed after tamoxifen therapy [18]. 
The Intergroup Exemestane Study (IES) was designed to investigate whether exemestane, when given to postmenopausal women who remained free of recurrence after receiving adjuvant tamoxifen therapy for two to three years for primary breast cancer, could prolong disease-free survival, as compared with continued tamoxifen therapy. Here we report the results of the second planned interim analysis, which we are releasing in accordance with the recommendation of the independent data and safety monitoring committee.

\section{Methods}

Study Design. Our study is an international, intergroup, phase 3, randomized, doubleblind trial comparing the efficacy and safety of continued adjuvant tamoxifen therapy with the efficacy and safety of exemestane therapy in postmenopausal women with primary breast cancer who remain free of disease after receiving adjuvant tamoxifen therapy for two to three years. Women were randomly assigned to receive oral exemestane $(25 \mathrm{mg})$ or tamoxifen $(20 \mathrm{mg})$ daily in order to complete a total of five years of adjuvant endocrine treatment (Figure 1). Randomization was performed with the use of permuted blocks and was stratified according to center.

The primary end point was disease-free survival, defined by the time from randomization to recurrence of breast cancer at any site, diagnosis of a second primary breast cancer, or death from any cause. Secondary end points included overall survival, the incidence of contralateral breast cancer, and long-term tolerability. For consistency and comparability with other reported trials [19], we also report breast-cancer-free survival, with censoring of deaths that occurred without a recurrence of breast cancer or a diagnosis of contralateral breast cancer. Results from substudies assessing the quality of life, uterine thickness, bone metabolism, and bone mineral density will be reported separately.

The study was coordinated by the International Collaborative Cancer Group (ICCG), Imperial College London, and conducted under the auspices of the Breast International Group (BIG). The trial was governed by a steering committee comprising representatives from the ICCG, participating cooperative groups, BIG, and the pharmaceuticalindustry sponsor. Data for each cooperative group were collected by the group's data center and collated centrally by the ICCG Data Center. Central review and querying and analysis of data were undertaken by the ICCG Data Center in collaboration with the Institute of Cancer Research, where the independent statisticians were based. The sponsor had no access to the trial data base or interim analyses. The study was overseen by a data and safety monitoring committee that was independent of the ICCG Data Center, the steering committee, and the sponsor. The institutional review board at each participating institution approved the study protocol, and all patients gave written informed consent. Randomization was performed by the data center for each cooperative group or through the ICCG Data Center. 
FIGURE 1

Trial Schema. The percentage of patients who continue to receive treatment represents the percentage who are not known to have discontinued their randomized treatment and who began initial tamoxifen therapy less than five years before December 31, 2003.

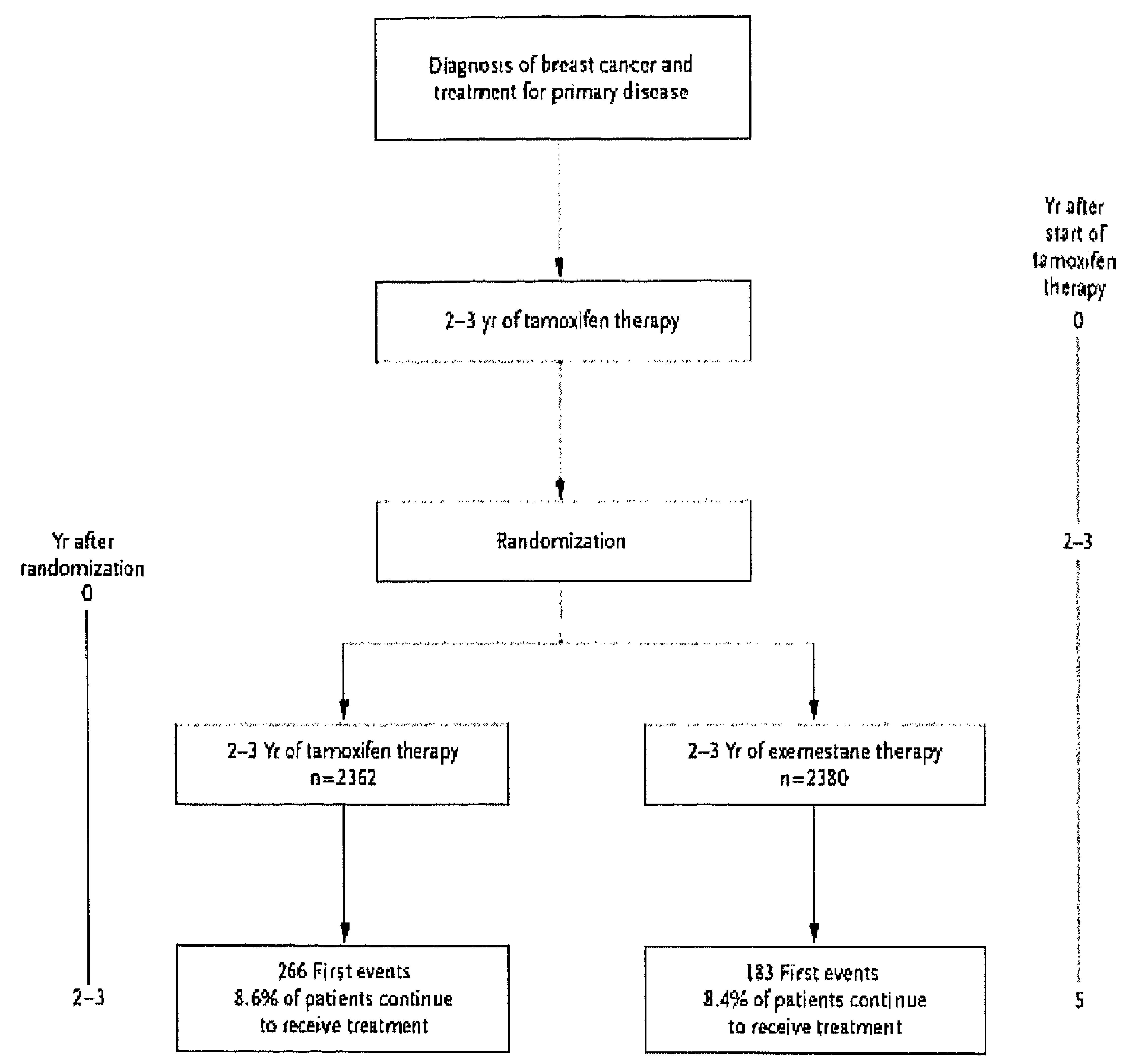

Eligibility Criteria. Patients were eligible if they had histologically confirmed, completely resected unilateral invasive breast carcinoma that was positive for estrogen receptors (as determined by means of standard immunostaining procedures) or that was of unknown receptor status. Patients were postmenopausal (55 years of age or older with amenorrhea for more than two years, or amenorrhea for more than one year at the time of diagnosis) and had received adjuvant tamoxifen therapy for at least two years but not more than three years and one month. Most patients ( 95 percent) received tamoxifen at a dose of $20 \mathrm{mg}$ daily, but patients who received $30 \mathrm{mg}$ daily were eligible (and continued to receive the same dose if they were assigned to the tamoxifen group). Patients were required to have adequate hematologic, renal, and liver function at the time of randomi- 
zation (defined as a normal blood count, a serum creatinine concentration less than 1.5 times the upper limit of normal, and a serum alanine aminotransferase concentration less than 2.5 times the upper limit of normal). The criteria for exclusion included the presence of a tumor with known negative estrogen-receptor status; evidence of local relapse or a distant metastasis since the time of diagnosis; a clinically significant skeletal, cardiac, or endocrine disorder; and the use of hormone-replacement therapy within four weeks before randomization. Patients were also excluded if they had clinical evidence of severe osteoporosis or a history of a previous neoplasm other than carcinoma in situ of the cervix or basal-cell skin carcinoma or if they were taking concomitant anticoagulant agents, a selective estrogen-receptor modulator other than tamoxifen, or any other form of hormonal therapy.

The protocol required adequate treatment of primary disease, including postoperative radiotherapy in patients who had been treated with breast-preserving surgery. Neoadjuvant chemotherapy was permitted according to a consistent policy within each center. Patients were required to have started chemotherapy within three months after diagnosis and to have begun receiving tamoxifen and radiotherapy within three months after the completion of chemotherapy.

Follow-up Procedures. Symptoms, side effects, findings on clinical examination, and the level of compliance with treatment were recorded at three-month intervals during the first year after randomization, every six months during the second and third years, and annually thereafter. Hematologic and biochemical analyses and mammography (if the local procedure permitted) were performed annually.

Statistical Analysis. Enrollment of 4400 patients was required in order to detectan absolute difference of 3.6 percent in disease-free survival three years after randomization (with 88 percent power and a two-sided level of significance of 4.3 percent after adjustment for interim analyses). The a priori expectation was that the principal analysis would be conducted after 716 end-point events had occurred. Three interim efficacy analyses were to be conducted, with the use of O'Brien-Fleming stopping boundaries, after one quarter, one half, and three quarters of the planned total number of events. Emerging trial data and interim analyses were reviewed by the independent data and safety monitoring committee, whose terms of reference dictated that their decisions be guided (but not mandated) by the above stopping rules. Analyses were performed according to the intention-to-treat principle and included all patients who underwent randomization. All data were censored on June 30, 2003, but the snapshot of data used for the analysis of efficacy was updated to include all data received by the ICCG Data Center through December 31, 2003. Log-rank tests were used to compare the two groups. Two-sided $P$ values and 95 percent confidence intervals are reported. Cox proportionalhazards regression was used to adjust for pre-specified prognostic factors [20]. Hazard ratios of less than 1.0 favor exemestane. Kaplan-Meier time-to-event curves are presented. The groups were compared in terms of the incidence of adverse effects with the use of chi-square tests. Because of the early release of the efficacy results, data on adverse events are provisional; the validation process is ongoing. Here, we emphasize the adverse effects for which there is a difference between groups with a $P$ value of 0.01 or less. 


\section{Results}

Study Population. We recruited 4742 women from 37 countries and 20 cooperative groups between February 1998 and February 2003. Recruitment continued beyond the target enrollment of 4400 in order to complete accrual to the substudies on the effects on bone and quality of life. The median follow-up was 30.6 months (interquartile range, 23.9 to 36.6 ). The two groups were balanced with regard to base-line characteristics (Table 1). A total of 192 patients were subsequently found to be ineligible ( 16 because

\begin{tabular}{|c|c|c|}
\hline Variable & Exeme stane $(N=2362)$ & Tamoxifen $(\mathrm{N}=2380)$ \\
\hline \multicolumn{3}{|l|}{ Demographic characteristics } \\
\hline Age - yr & $64.3+.8 .1$ & $64.2 \pm 8.2$ \\
\hline White race - no. (96) & $2308(97.7)$ & $2325(97.7)$ \\
\hline \multicolumn{3}{|l|}{ Nodal status - no. $(96)$} \\
\hline Negative & $1211(51.3)$ & $1211(50.9)$ \\
\hline 1-3 Pasitive nodes & $715(30.3)$ & $706(29.7)$ \\
\hline 24 Positive nodes & $321(13.6)$ & $330(13.9)$ \\
\hline Positive, but no. of nodes missing & $5(0.2)$ & $9(0,4)$ \\
\hline Unknown & $84(3.6)$ & $96(4.0)$ \\
\hline Missing data & $26(1.1)$ & $28(1.2)$ \\
\hline \multicolumn{3}{|l|}{ Histologic type - no. (\%) } \\
\hline Infiltrating ductal & $1814(76.8)$ & $1871(78.6)$ \\
\hline Infiltrating lobutar & $346(14.6)$ & $327(13.7)$ \\
\hline Other & $172 .(7.3)$ & $156(6.6)$ \\
\hline Unknown & $3(0.1)$ & $1(\leftarrow 0.1)$ \\
\hline Missing data & $27(1.1)$ & $25(1.1)$ \\
\hline \multicolumn{3}{|l|}{ Estrogen.receptor status - no. $(06) j$} \\
\hline Positive & $1917(81.2)$ & $1936(81.3)$ \\
\hline Progester one-receptor po sitive & $1312(55.6)$ & $130 \%(54.9)$ \\
\hline Progesterone-receptor negative & $351(14.9)$ & $384(16.1)$ \\
\hline Progester one-receptor status unknown or missing & $254(10.8)$ & $245(10.3)$ \\
\hline Negative & $26(1.1)$ & $33(1.4)$ \\
\hline Unknown & $398(16.9)$ & $392(16.5)$ \\
\hline Missing data & $21(0.9)$ & $19(0.8)$ \\
\hline \multicolumn{3}{|l|}{ Progest erone-receptor status - no. (\%) } \\
\hline Positive & $1320(55.9)$ & $1313(55.2)$ \\
\hline Negative & $360(15.2)$ & $395(16.6)$ \\
\hline Unknown & $659(27.9)$ & $653(27.4)$ \\
\hline Missing data & $23(1.0)$ & $19(0.8)$ \\
\hline \multicolumn{3}{|l|}{ Type of surgery - no. (\%) } \\
\hline Mastectomy & $1222(51.7)$ & $1235(51.9)$ \\
\hline Breast-conserving & $1116(47.2)$ & $1123(47.2)$ \\
\hline Unknown & $3(0.1)$ & $2(0.1)$ \\
\hline Missing data & $21(0.9)$ & $20(0.8)$ \\
\hline \multicolumn{3}{|l|}{ Previous cherriotherapy - no. $(\%)$} \\
\hline Yes & $766(32.4)$ & $765(32.1)$ \\
\hline No & $1575(66.7)$ & $1596(67.1)$ \\
\hline Missing data & $21(0.9)$ & $19(0.8)$ \\
\hline \multicolumn{3}{|l|}{ Previous hormone.r eplacement therapy - no. (96) } \\
\hline Yes & $567(24.0)$ & $557(23.4)$ \\
\hline No & $1723(72.9)$ & $1747(73.4)$ \\
\hline Unknown & $51(2.2)$ & $54(2.3)$ \\
\hline Missing data & $21(0.9)$ & $22(0.9)$ \\
\hline \multicolumn{3}{|l|}{ Duration of tamoxifen therapy at randomization - yr } \\
\hline Median & 2.4 & 2.4 \\
\hline Interquartlle range & $2.1-2.7$ & $2.1-2.7$ \\
\hline \multicolumn{3}{|l|}{ Tamoxifen dose - no. (96) } \\
\hline $20 \mathrm{mg}$ & $2243(95.0)$ & $2270(95.4)$ \\
\hline $30 \mathrm{mg}$ & $77(3.3)$ & $76(3.2)$ \\
\hline Missing data & $42(1.8)$ & $34(1.4)$ \\
\hline $\begin{array}{l}\text { *Plus-minus values are means } \pm S D \text {. Patients with missing } \\
\text { the "unknown" category, data were reported as unknown. } \\
\text { Data for positive and negative estrogen-receptor status in } \\
\text { whose status was unknown at randomization. }\end{array}$ & $\begin{array}{l}\text { no value reported for a gi } \\
\text { ospectively ascertained s }\end{array}$ & $\begin{array}{l}\text { ariable; for patients in } \\
\text { for some patients }\end{array}$ \\
\hline
\end{tabular}


of previous breast cancers, 31 because of previous other cancers, 74 because they had undergone breast-conserving surgery but had not received radiotherapy, 25 because they were of uncertain menopausal status, 24 because they had known estrogen-receptornegative tumors, 8 because they had used hormone-replacement therapy within four weeks before randomization, and 14 for other reasons); these patients are included in all analyses on an intention-to-treat basis.

Efficacy. The second interim analysis, which was triggered by the reporting of 358 events, was presented to the data and safety monitoring committee on December 2, 2003, and included all data that had been received relating to events and follow-up through June 30,2003 . At that meeting, the committee recommended that key efficacy data be released, because the $\mathrm{O}^{\prime} B$ rien-Fleming stopping boundary $(\mathrm{P}=0.004)$ had been exceeded. The steering committee agreed to the release at a meeting on December 3,

\begin{tabular}{|c|c|c|c|}
\hline \multirow[t]{2}{*}{ Variable } & $\begin{array}{l}\text { Exemestane Group } \\
\qquad(\mathrm{N}=2362)\end{array}$ & $\begin{array}{l}\text { Tarnoxifen Group } \\
\qquad(\mathrm{N}=2380)\end{array}$ & $\begin{array}{l}\text { All Patients } \\
(N=4742)\end{array}$ \\
\hline & \multicolumn{3}{|c|}{ no. of patients } \\
\hline \multicolumn{4}{|l|}{ Events included in analys is of disea se.free survival" } \\
\hline Local recurrence only & 21 & 33 & 54 \\
\hline Distant recurrence & 114 & 174 & 288 \\
\hline Primary cancer in contralateral breast & 9 & 20 & 29 \\
\hline Intercurrent death (without recurrence) & 39 & 39 & 78 \\
\hline Recurrence, contralater al breast cancer, or intercurrent death & 183 & 266 & 449 \\
\hline \multicolumn{4}{|l|}{ Death } \\
\hline Any cause & 93 & 106 & 199 \\
\hline Breast-cancer-related & 54 & 67 & 121 \\
\hline Intercurrent (without recurrence) & 39 & 39 & 78 \\
\hline Vascular causes & 12 & 6 & 18 \\
\hline Cardiac causes & 10 & 8 & 18 \\
\hline Other cancer-related & 6 & 10 & 16 \\
\hline Thrombotic causes & 1 & 1 & 2 \\
\hline Pulmonary causes & 0 & 1 & 1 \\
\hline Other causes & 6 & 7 & 13 \\
\hline Unknown causes or missing data & 4 & 6 & 10 \\
\hline Second primary non-breast cancer & 27 & 53 & 80 \\
\hline Lung & 4 & 12 & 16 \\
\hline Gastrointestinal & 7 & 10 & 17 \\
\hline Endometrial & 5 & 11 & 16 \\
\hline Other gynecologic & 3 & 3 & 6 \\
\hline Genitourinary & 3 & 4 & 7 \\
\hline Melanoma & 1 & $s$ & 6 \\
\hline Lymphoreticular & 2 & 3 & 5 \\
\hline Other & 2 & 5 & 7 \\
\hline
\end{tabular}

* Data for distant recurrence and primary cancer in the contralateral breast include patients who also reported a local relapse. One patient in the exemestane group died of breast cancer after contralateral breast cancer had been reported and is also included in the analysis of distant-disease-free survival. 
2003. This report constitutes a refined analysis of that presented to the data and safety monitoring committee.

FIGURE 2

Kaplan-Meier Estimates of Disease-free Survival (Panel A) and Overall Survival (Panel B). The hazard ratios are for the exemestane group as compared with the tamoxifen group. P values were determined by the log-rank test. An additional six patients in the exemestane group and four patients in the tamoxifen group had a recurrence or a second primary cancer in the contralateral breast or died more than four years after randomization (Panel A); an additional four patients in the exemestane group and two patients in the tamoxifen group died more than four years after randomization (Panel B).

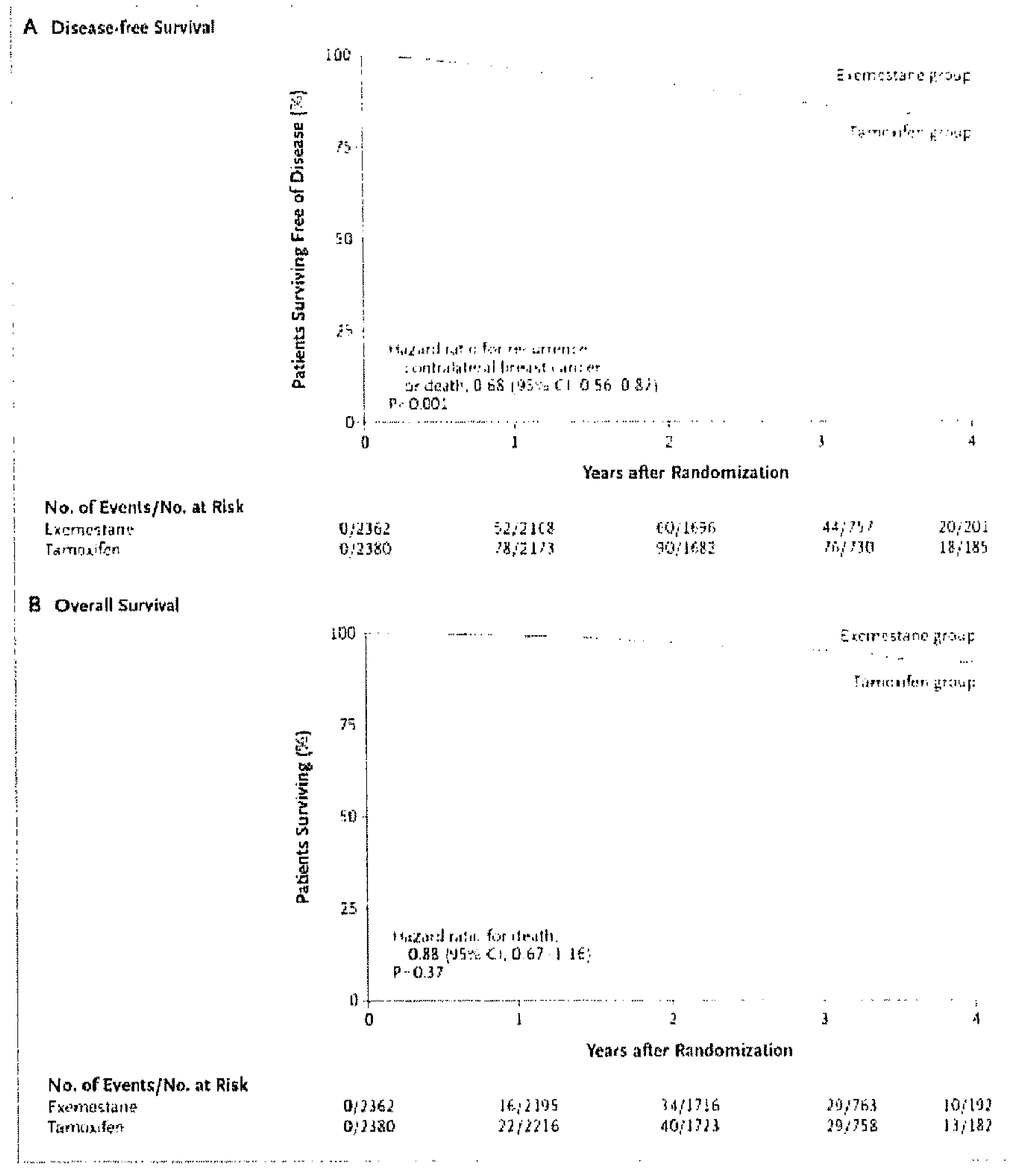

A total of 449 first events were reported: 183 in the exemestane group and 266 in the tamoxifen group (Table 2). The unadjusted hazard ratio in the exemestane group as 
compared with the tamoxifen group was 0.68 (95 percent confidence interval, 0.56 to $0.82 ; \mathrm{P}=0.00005$ by the log-rank test), which corresponds to an absolute benefit of 4.7 percent (95 percent confidence interval, 2.6 to 6.8 ) at three years (Figure 2). Diseasefree survival three years after randomization was 91.5 percent $(95$ percent confidence interval, 90.0 to 92.7 ) in the exemestane group and 86.8 percent ( 95 percent confidence interval, 85.1 to 88.3 ) in the tamoxifen group. In a subsidiary analysis of breast-cancerfree survival in which deaths of patients who did not have a recurrence or contralateral breast cancer were censored, the hazard ratio was 0.63 (95 percent confidence interval, 0.51 to $0.77 ; \mathrm{P}=0.00001 ; 144$ events in the exemestane group vs. 227 in the tamoxifen group). Survival free of distant disease was also better in the exemestane group (hazard ratio, $0.66 ; 95$ percent confidence interval, 0.52 to $0.83 ; \mathrm{P}=0.0004)$. A total of 199 patients have died (93 in the exemestane group and 106 in the tamoxifen group). There is no statistically significant difference in overall survival at this stage (hazard ratio, 0.88 ; 95 percent confidence interval, 0.67 to $1.16 ; \mathrm{P}=0.37$ ) (Figure 2). The causes of death are listed in Table 2. Exemestane significantly reduced the risk of contralateral breast cancer (hazard ratio, $0.44 ; 95$ percent confidence interval, 0.20 to $0.98 ; \mathrm{P}=0.04$ ).

Adjusting for the pre-specified prognostic factors did not affect the hazard ratios (Table 3), and there was no evidence of heterogeneity among subgroups defined according to estrogen-receptor status, combined estrogen-receptor and progesterone-receptor status, number of positive nodes, receipt or type of previous chemotherapy, or use at any time of hormone-replacement therapy (Figure 3).

\begin{tabular}{|c|c|c|c|c|}
\hline \multicolumn{5}{|c|}{$\begin{array}{l}\text { Table } 3 . \text { Hazard Ratios in the Exemestane Group as Compared } \\
\text { with the Tamoxifen Group. }\end{array}$} \\
\hline \multirow[t]{2}{*}{ End Point } & \multicolumn{2}{|c|}{ Unadjusted } & \multicolumn{2}{|l|}{ Adjusted } \\
\hline & $\begin{array}{c}\text { Hazard Ratio } \\
(95 \% \mathrm{Cl})\end{array}$ & PValue & $\begin{array}{c}\text { Hazard Ratio } \\
(95 \% \mathrm{Cl})\end{array}$ & PValue \\
\hline Dis ease-free survival & $0.68(0.56-0.82)$ & $<0.001$ & $0.67(0.56-0.82)$ & $<0.001$ \\
\hline $\begin{array}{l}\text { Breast-cancer-free } \\
\text { survival }\end{array}$ & $0.63(0.51-0.77)$ & $<0.001$ & $0.62(0.50-0.76)$ & $<0.001$ \\
\hline $\begin{array}{l}\text { Time to contralateral } \\
\text { breast cancer }\end{array}$ & $0.44(0.20-0.98)$ & 0.04 & $0.44(0.20-0.98)$ & 0.04 \\
\hline Overall survival & $0.88(0.67-1.16)$ & 0.37 & $0.89(0.67-1.17)$ & 0.41 \\
\hline \multicolumn{5}{|c|}{$\begin{array}{l}\text { A Cox model including estrogen-receptor status (either positive or negative, } \\
\text { unknown or missing), nodal status (n egative, } 1 \text { to } 3 \text { positive nodes, } 4 \text { or more } \\
\text { positive nodes, or unknown or missing), chemotherapy (yes or no), and use of } \\
\text { hormone-replacement therapy (yes, no, or unknown or mis sing) was used to } \\
\text { estimate the adjusted hazard ratios. Forty patients with unknown chemother- } \\
\text { apy status were excluded from the analysis. Cl denotes confidence interval. } \\
\text { P values were determined by the log-rank test. }\end{array}$} \\
\hline
\end{tabular}




\section{FIGURE 3}

Subgroup Analysis of Disease-free Survival. The hazard ratio given for all patients was adjusted for estrogen-receptor status, nodal status, receipt or non-receipt of chemotherapy, and use or nonuse of hormone-replacement therapy $(\mathrm{P}=0.00004)$. The size of the rectangles is proportional to the size of the subgroups.

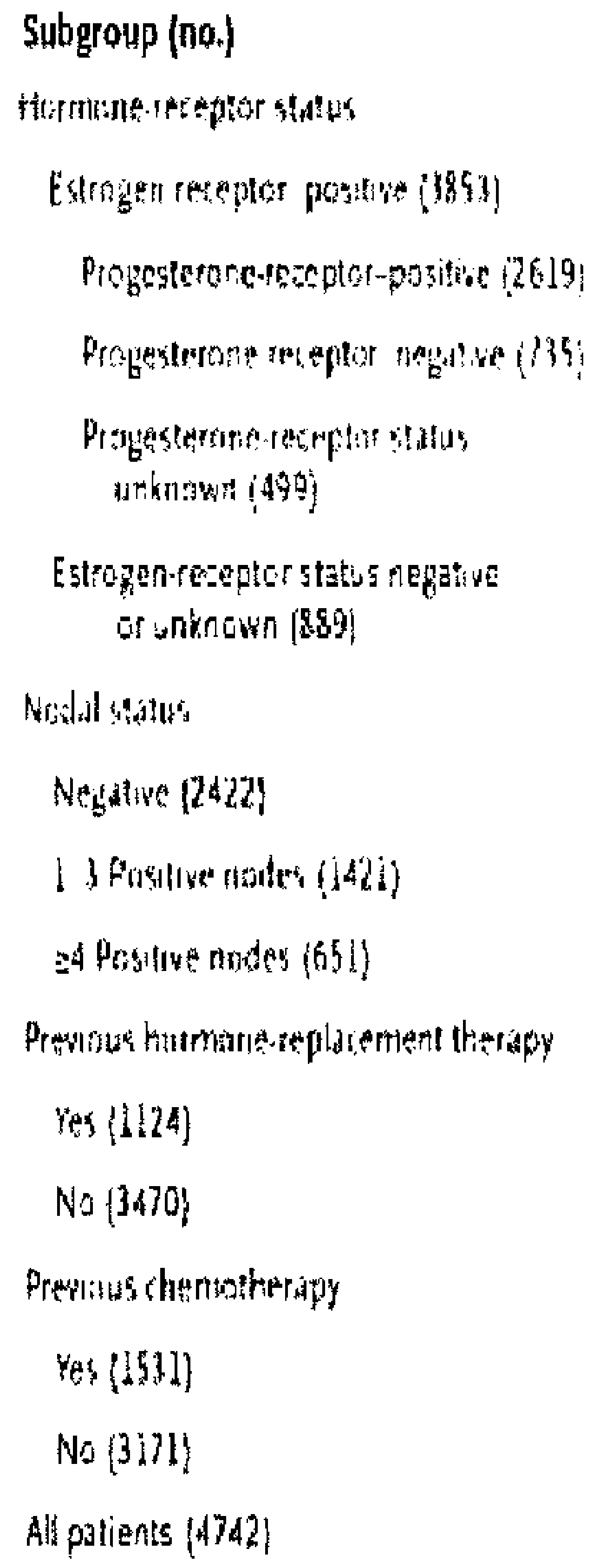

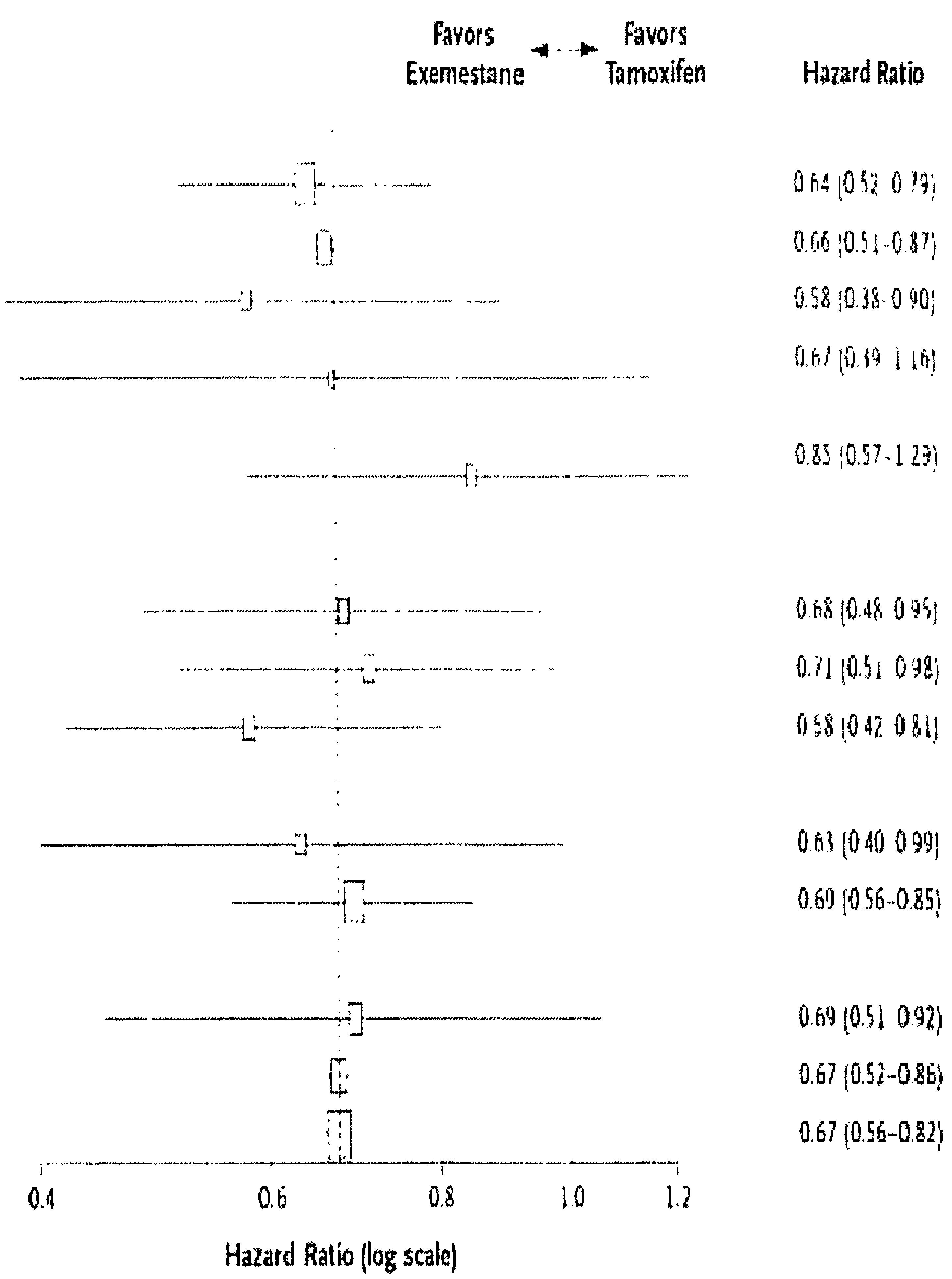

Adverse Effects and Safety. Exemestane was associated with a higher incidence of arthralgia and diarrhea than tamoxifen, but gynecologic symptoms, vaginal bleeding, and muscle cramps were more common with tamoxifen (Table 4). Thromboembolic events were recorded more frequently in the tamoxifen group than in the exemestane group ( 55 patients [2.4 percent] vs. 30 patients [1.3 percent], $\mathrm{P}=0.007$ ). There was also a suggestion of an increased incidence of osteoporosis and visual disturbances associated with exemestane. Fractures were reported more frequently in the exemestane group than in the tamoxifen group, although the difference was not statistically significant (72 patients [ 3.1 percent] vs. 53 patients [2.3 percent], $\mathrm{P}=0.08$ ). More patients in the tamoxifen group than in the exemestane group had a second primary non-breast cancer that occurred before a distant relapse (53 patients [2.2 percent] vs. 27 patients [1.1 percent]; 
hazard ratio, $0.51 ; 95$ percent confidence interval, 0.32 to $0.80 ; \mathrm{P}=0.003$ ) (Table 2). Specifically, cancer of the endometrium, lung cancer, and melanoma developed in fewer patients in the exemestane group than in the tamoxifen group, although these individual differences were not statistically significant.

\begin{tabular}{|c|c|c|c|c|c|c|c|c|c|c|c|}
\hline \multicolumn{12}{|l|}{ Table 4, Adverse Events." } \\
\hline \multirow[t]{4}{*}{ Type of Event } & \multicolumn{5}{|c|}{ Exemestane Group } & \multicolumn{5}{|c|}{ Tamoxifen Group } & \multirow[t]{3}{*}{ PValue } \\
\hline & Grade & Grade & Grade & Grade & & Grade & Grade & Grade & Grade & & \\
\hline & 1 & 2 & 3 & 4 & Any Grade & 1 & 2 & 3 & 4 & Any Grade & \\
\hline & \multicolumn{10}{|c|}{ number (percent) } & \\
\hline $\begin{array}{l}\text { Cardiovascular disease other than } \\
\text { myocardial infarction }\end{array}$ & & & & & $984(42.6)$ & & & & & $913(39.2)$ & 0.016 \\
\hline Hot llashes & 504 & 363 & 97 & 3 & $967(42.0)$ & 493 & 342 & 84 & 4 & $923(39.6)$ & 0.082 \\
\hline Pain or aches & 392 & 305 & 61 & 8 & $766 \mid 33.2\}$ & 383 & 242 & 35 & 4 & $684(29.4)$ & 0.001 \\
\hline Fatgue & 336 & 178 & 31 & 0 & $545(23.6)$ & 352 & 157 & 36 & 2 & $547(23.5)$ & 0.176 \\
\hline Insomnia & 269 & 143 & 37 & 0 & $449(19.5)$ & 234 & 140 & 31 & 1 & $406(17.4\}$ & 0.151 \\
\hline Sweating & 222 & 153 & 51 & 3 & $429\{18.6\}$ & 215 & 145 & $\$ 1$ & 1 & $418(17.9)$ & 0.702 \\
\hline Headaches & 272 & 129 & 26 & 1 & $428(18.6)$ & 243 & 116 & 17 & 2 & $378(16.2)$ & 0.035 \\
\hline Dizziness & 206 & 73 & 9 & 0 & $288(12.5)$ & 192 & 74 & 13 & 0 & $279(12.0)$ & 0.904 \\
\hline Nausea & 177 & 57 & 14 & 0 & $248(10.8)$ & 189 & 53 & 16 & 0 & $258(11.1)$ & 0.835 \\
\hline Visual disturbances & 134 & 32 & 4 & 0 & $170(7.4)$ & 115 & 8 & 10 & 0 & $133(5.7)$ & 0.024 \\
\hline Osteoporosis & & & & & $171(7.4)$ & & & & & $134(5.7)$ & 0.023 \\
\hline Gynecologic symptoms & & & & & $135(5.8)$ & & & & & $211(9.0)$ & $<0.001$ \\
\hline Arthralgia & & & & & $124(5.4)$ & & & & & $85(3.6)$ & 0.005 \\
\hline Depression & 68 & 50 & 2 & 0 & $120(5.2)$ & $\$ 1$ & 37 & 5 & 0 & $93(4.0)$ & 0.114 \\
\hline Diarrhéa & 63 & 28 & 8 & 1 & $100(4.3)$ & 37 & 16 & 1 & 0 & $54(2.3)$ & $<0.001$ \\
\hline Vaginal bleeding & 49 & 33 & 11 & 0 & $93(4.0)$ & 73 & 50 & 5 & 1 & $129(5.5)$ & 0.087 \\
\hline Cramps & 45 & 16 & 3 & 0 & $64(2.8)$ & 60 & 37 & 3 & 2 & $102(4.4)$ & 0.002 \\
\hline $\begin{array}{l}\text { Thromboembolic disease } \\
\text { Including ungraded serious } \\
\text { adverse events }\end{array}$ & 11 & 4 & 8 & 1 & $\begin{array}{l}24(1.0) \\
30(1.3)\end{array}$ & 11 & 13 & 15 & 6 & $\begin{array}{l}45(1.9) \\
55(2.4)\end{array}$ & $\begin{array}{l}0.005 \\
0.007\end{array}$ \\
\hline
\end{tabular}

* Data are given for adverse events whose incidence in the two groups differed by 1 percent or more, for which the difference between groups was significant at the I percent level, or whose incidence was at least 10 percent in either group. Grades are according to Common Toxicity Criteria of the National Cancer Institute (version 1.0). Data on cardiovascular disease, gynecologic symploms, osteoporosis, and arthralgia were available for 2309 patients in the exemestane group and 2332 patients in the tamoxifen group; data on the other adverse effects were avalable for 2305 and 2329 patients, respectively. Pain or aches, arthralgia, depression, diarrhea, and cramps were recorded in an "other" caregory; data are preliminary and may underestimate the true incidence. For graded adverse events, $P$ values were determined by trend tesis combin. ing grades 3 and 4 . 
Treatment Compliance. Randomly assigned treatment was stopped early in 667 patients (365 in the exemestane group and 302 in the tamoxifen group; 14.1 percent of the total study population) for reasons other than relapse or death, after a median total duration of treatment of 36.1 months (from the initiation of tamoxifen therapy). A total of 138 patients in the exemestane group and 121 in the tamoxifen group discontinued therapy because of adverse events, and another 164 patients in the exemestane group and 116 in the tamoxifen group refused to continue therapy. An additional 63 patients in the exemestane group and 65 in the tamoxifen group have discontinued their randomly assigned treatment for other reasons, including protocol violations, or have been lost to follow-up. On the basis of the time since randomization, 9 percent of patients are likely to be still receiving treatment.

\section{Discussion}

We found that switching patients to adjuvant treatment with exemestane after two to three years of tamoxifen therapy was associated with a statistically and clinically significant improvement in disease-free survival, which included a reduction in the incidence of metastatic disease. This strategy also reduced the risks of contralateral breast cancer, endometrial cancer, and intriguingly, other primary cancers. At the time of this report, the observed number of deaths over the relatively short follow-up period precludes the detection of a statistically significant difference in overall survival. The data and safety monitoring committee recommended the early release of results on the basis of a planned interim analysis. More than 90 percent of the patients will have completed their randomly assigned treatment by the time this report is published; thus, the trial should still be able to achieve its long-term assessment of survival benefit. There are several theoretical reasons to suggest a benefit of sequential endocrine therapy involving switching from tamoxifen to an aromatase inhibitor after two to three years. First, many patients with breast cancer have a relapse and die of metastatic disease within five years after the initial diagnosis. Second, in both patients with primary cancer and those with metastatic disease, resistance occurs as early as 12 to 18 months after the initiation of tamoxifen therapy. In some patients with resistant disease, tamoxifen may act as an agonist, potentially stimulating the division of breast-cancer cells. Third, serious side effects of tamoxifen, including thromboembolism and uterine carcinoma, can occur after prolonged use. Fourth, since tamoxifen can decrease bone resorption [21], we reasoned that pretreatment with tamoxifen might lessen the effect of any osteopenia caused by exemestane.

When we designed this study, there was considerable uncertainty regarding the optimal duration of adjuvant tamoxifen therapy in patients with primary breast cancer. The 1990 overview by the Early Breast Cancer Trialists' Collaborative Group had suggested that there was a likely benefit of continuing tamoxifen therapy for five years [22]. Randomized trials directly comparing two years of tamoxifen therapy with five years of tamoxifen therapy $[23,24]$ confirmed that there was a relative risk reduction of 18 to 19 percent with the longer-term therapy. Thus, although five years of tamoxifen treatment was the identified standard, switching treatment after only two to three years was postulated to offer patients the bulk of the benefit of tamoxifen while minimizing the risk of long-term side effects. 
Despite the promising results of the Anastrozole, Tamoxifen Alone or in Combination Trialists' Group (ATAC) study, which showed that anastrozole was superior to tamoxifen [25], five years of tamoxifen therapy remains the widely recommended standard for adjuvant treatment [8], although the Food and Drug Administration recently approved anastrozole monotherapy as an alternative. A study by Goss et al. [19] found that after five years of tamoxifen therapy, patients who received letrozole had a higher rate of disease-free survival than those who received placebo. Our large, multicenter study challenges the concept of five years of monotherapy with endocrine agents after the surgical treatment of primary breast cancer. Two smaller studies conducted by Italian researchers have used sequential aminoglutethimide after tamoxifen therapy in 308 patients [26] and anastrozole after tamoxifen therapy in 426 patients [27]. Although they were underpowered, both trials suggested that the sequence may be better than tamoxifen alone, supporting the results we present here.

The improvement in disease-free survival achieved by switching from tamoxifen to exemestane is consistent with the hypothesis that breast cancer frequently becomes resistant to tamoxifen within five years after treatment is initiated. The molecular mechanisms underlying such resistance are unclear. Laboratory studies indicate that a reduction in the antagonist properties of tamoxifen caused by the up-regulation of tyrosine kinase receptors (in particular, HER2 and epidermal growth-factor receptors), downstream protein kinases (such as mitogen-activated protein kinase [28] and protein kinase B, or Akt [29]), or both may result in a significant increase in the agonist activity of tamoxifen, as well as increased sensitivity to estradiol. These effects could explain the benefit that has been observed to result from lowering the estradiol level through the sequential use of an aromatase inhibitor [9].

Results in the subgroup with estrogen-receptor-positive breast cancer were very similar to those among all patients. According to an unplanned subgroup analysis, exemestane seemed to be equally effective in both progesterone-receptor-positive and progesteronereceptor-negative subgroups, as well as in node-positive and node-negative subgroups, contrary to the report suggesting that patients with estrogen-receptor-positive and progesterone-receptor-negative carcinomas may preferentially benefit from anastrozole therapy [30].

The reduction in the incidence of contralateral breast cancer in the exemestane group as compared with the tamoxifen group (hazard ratio, $0.44 ; 95$ percent confidence interval, 0.20 to $0.98 ; \mathrm{P}=0.04$ ) suggests that preventive strategies involving the prolonged use of tamoxifen monotherapy [31,32] may not be optimal. The non-significant decrease in the rate of endometrial cancer is consistent with expectations, since tamoxifen therapy is a well-recognized risk factor for endometrial cancer $[33,34]$. The decreased incidence of other second primary (non-breast) cancers is more difficult to explain. Reports of associations between tamoxifen therapy and cancer at other sites have been inconclusive [34], and such associations were not substantiated by the Early Breast Cancer Trialists' Collaborative Group study [5]. Thus, it is not clear whether the observed differences in the incidence of new primary cancers represent increases in risks due to tamoxifen treatment, a previously unreported protective effect of an aromatase inactivator; or chance findings.

The rate of discontinuation of treatment was slightly higher in the exemestane group than in the tamoxifen group, perhaps reflecting differences in the side-effect profiles of the two treatments that may have been particularly evident to patients switching from 
one treatment to another. The analysis of adverse events indicated that there was a lower incidence of thromboembolic events among women who switched to exemestane. There was a slight but non-significant increase in the rate of osteoporosis and reported fractures in the exemestane group as compared with the tamoxifen group. Recent studies have shown that all third-generation aromatase inhibitors or inactivators increase bone resorption $[35,36]$. The substudy of the IES on bone mineral density aims to determine the degree of bone mineral loss in patients who have been treated with tamoxifen and then switched to exemestane. The increase in the rate of arthralgia in the exemestane group is similar to that seen with other aromatase inhibitors [37], and diarrhea has been reported previously in patients receiving exemestane [16]. Cholesterol levels, which were reduced by tamoxifen treatment [38], were found to be unaltered in another study of exemestane [39] but were not systematically measured in the present study; we have not observed a significantly increased incidence of myocardial infarction ( 1.0 percent in the exemestane group vs. 0.4 percent in the tamoxifen group).

Several issues still need to be clarified, including the correct sequence of therapy, which we believe to be an important factor in the success of this study and that reported by Goss et al. [19], as well as the effect of aromatase inhibition on bone metabolism. The answers to these questions will have to await the results of ongoing and new studies. Our results add to the evidence that the sequential use of aromatase inactivators and tamoxifen provides additional options for improving adjuvant endocrine therapy for postmenopausal women with hormone-responsive primary breast cancer. Our results indicate that five years of tamoxifen monotherapy after surgery may be sub optimal for postmenopausal patients with estrogen-receptor-positive breast cancer and suggest that clinicians should consider switching patients to exemestane between two and three years after the start of tamoxifen therapy.

\section{References}

1. Beatson GT. On the new treatment of inoperable cases of carcinoma of the mamma: suggestions for a new method of treatment, with illustrative cases. Lancel 1896;2:104-107.

2. Jensen EV, Jordan VC. The estrogen receptor: a model for molecular medicine. Clin Cancer Res 2003;9:1980-1989.

3. Cole MP, Jones CTA, Todd IDH. A new anti-oestrogenic agent in late breast cancer: an early clinical appraisal of IC46474. Br J Cancer 1971;25:270-275.

4. Jordan VC. Third annual William L. McGuire Memorial Lecture: "studies on the estrogen receptor in breast cancer" -20 years as a target for the treatment and prevention of cancer. Breast Cancer Res Treat 1995;36:267-285.

5. Early Breast Cancer Trialists' Collaborative Group. Tamoxifen for early breast cancer: an overview of the randomised trials. Lancet 1998;351:1451-1467.

6. Peto R. Five years of tamoxifen - or more? J Natl Cancer Inst 1996;88:1791-1793.

7. Fisher B, Dignam J, Bryant J, et al. Five versus more than five years of tamoxifen therapy for breast cancer patients with negative lymph nodes and estrogen-receptor positive tumors. $J$ Natl Cancer Inst 1996;88:1529-1542.

8. Goldhirsh A, Wood WC, Gelber RD, Coates AS, Thürlimann B, Senn H-J. Meeting highlights: updated international expert consensus on the primary therapy of early breast cancer. J Clin Oncol 2003;21:3357-3365. 
9. Ali S, Coombes RC. Endocrine-responsive breast cancer and strategies for combating resistance. Nat Rev Cancer 2002;2:101-112.

10. Buzdar AU, Jonat W, Howell A, et al. Anastrozole versus megestrol acetate in the treatment of postmenopausal women with advanced breast carcinoma: results of a survival update based on a combined analysis of data from two mature phase III trials. Cancer 1998;83:1 1421152. [Erratum, Cancer 1999;85:1010.

11. Dombernowsky P, Smith I, Falkson G, et al. Letrozole, a new oral aromatase inhibitor for advanced breast cancer: double-blind randomized trial showing a dose effect and improved efficacy and tolerability compared with megestrol acetate. J Clin Oncol 1998;16:453-461.

12. Evans TR, Di Salle E, Ornati G, et al. Phase I and endocrine study of exemestane (FCE 24304), a new aromatase inhibitor, in postmenopausal women. Cancer Res 1992;52:59335939.

13. Giudici D, Ornati G, Briatico G, Buzzetti F, Lombardi P, di Salle E. 6-Methylenandrosta1,4-diene-3,17-dione (FCE 24304): a new irreversible aromatase inhibitor. J Steroid Biochem 1988;30:391-394.

14. Hamilton A, Piccart $M$. The third-generation non-steroidal aromatase inhibitors: a review of their clinical benefits in the second-line hormonal treatment of advanced breast cancer. Ann Oncol 1999; 10:377-384.

15. Geisler J, King N, Anker G, et al. In vivo inhibition of aromatization by exemestane, a novel irreversible aromatase inhibitor, in postmenopausal breast cancer patients. Clin Cancer Res 1998;4:2089-2093.

16. Lonning PE, Bajetta E, Murray R, et al. Activity of exemestane in metastatic breast cancer after failure of nonsteroidal aromatase inhibitors: a phase II trial. J Clin Oncol 2000;18:22342244.

17. Paridaens R, Dirix L, Lohrisch C, et al. Mature results of a randomized phase II multicenter study of exemestane versus tamoxifen as first-line hormone therapy for postmenopausal women with metastatic breast cancer. Ann Oncol 2003;14:1391-1398.

18. Goldstein SR. The effect of SERMS on the endometrium. Ann $N$ Y Acad Sci 2001;949:237-242.

19. Goss PE, Ingle JN, Martino $S$, et al. A randomized trial of letrozole in postmenopausal women after five years of tamoxifen therapy for early-stage breast cancer. $\mathrm{N}$ Engl J Med 2003;349:1793-1802.

20. Cox DR. Regression models and life-tables. J R Stat Soc [B] 1972;34:187-220.

21. Love RR, Mazess RB, Barden HS, et al. Effects of tamoxifen on bone mineral density in postmenopausal women with breast cancer. N Engl J Med 1992;326:852-856.

22. Early Breast Cancer Trialists' Collaborative Group. Systemic treatment of early breast cancer by hormonal, cytotoxic, or immune therapy: 133 randomised trials involving 31000 recurrences and 24000 deaths among 75000 women. Lancet 1992;339:71-85.

23. Current Trials Working Party of the Cancer Research Campaign Breast Cancer Trials Group. Preliminary results from the cancer research campaign trial evaluating tamoxifen duration in women aged lifty years or older with breast cancer. J Natl Cancer Inst 1996;88:1834-1839. [Erratum, J Natl Cancer Inst 1997;89:590.]

24. Swedish Breast Cancer Cooperative Group. Randomized trial of two versus five years of adjuvant tamoxifen for postmenopausal early stage breast cancer. J Natl Cancer Inst 1996;88:1543-1549. 
25. Baum M, Budzar AU, Cuzick J, et al. Anastrozole alone or in combination with tamoxifen versus tamoxifen alone for adjuvant treatment of postmenopausal women with early breast cancer: first results of the ATAC randomised trial. Lancet 2002;359:2131-2139. [Erratum, Lancet 2002;360:1520.

26. Boccardo F, Rubagotti A, Amoroso D, et al. Sequential tamoxifen and aminoglutethimide versus tamoxifen alone in the adjuvant treatment of postmenopausal breast cancer patients: results of an Italian cooperative study. J Clin Oncol 2001;119:4209-4215.

27. Boccardo F, Rubagotti A, Amoroso D, et al. Anastrozole appears to be superior to tamoxifen in women already receiving adjuvant tamoxifen treatment. Breast Cancer Res Treat 2003;82:Suppl 1:S6-S6. abstract.

28. Chen D, Washbrook E, Sarwar N, et al. Phosphorylation of human estrogen receptor alpha at serine 118 by two distinct signal transduction pathways revealed by phosphorylation-specific antisera. Oncogene 2002;21:4921-4931.

29. Campbell RA, Bhat-Nakshatri P, Patel NM, Constantinidou D, Ali S, Nakshatri H. Phosphatidylinositol 3-kinase/AKT-mediated activation of estrogen receptor alpha: a new model for anti-estrogen resistance. J Biol Chem 2001;276:9817-9824.

30. Dowsett $M$. Analysis of time to recurrence in the ATAC (arimidex, tamoxifen, alone or in combination) trial according to estrogen receptor and progesterone receptor status. Breast Cancer Res Treat 2003;82:Suppl 1:S7-S7. abstract.

31. Fisher B, Costantino JP, Wickerham DL, et al. Tamoxifen for prevention of breast cancer: report of the National Surgical Adjuvant Breast and Bowel Project P-1 Study. J Natl Cancer Inst 1998;90:1371-1388.

32. Cuzick J, Powles $T$, Veronesi $U$, et al. Overview of the main outcomes in breast-cancer prevention trials. Lancet 2003;361:296-300.

33. Fisher B, Costantino JP, Redmond CK, Fisher ER, Wickerham DL, Cronin WM. Endometrial cancer in tamoxifen-treated breast cancer patients: findings from the National Surgical Adjuvant Breast and Bowel Project (NSABP) B-14. J Natl Cancer Inst 1994;86:527-537.

34. Rutqvist LE, Johansson H, Signomklao T, Johansson U, Fornander T, Wilking N. Adjuvant tamoxifen therapy for early stage breast cancer and second primary malignancies. J Natl Cancer Inst 1995;87:645-651.

35. Bajetta $\mathrm{E}$, Martinetti A, Zilembo $\mathrm{N}$, et al. Biological activity of anastrozole in postmenopausal patients with advanced breast cancer: effects on estrogens and bone metabolism. Ann Oncol 2002;13:1059-1066.

36. Harper-Wynne C, Ross G, Sacks N, et al. Effects of the aromatase inhibitor letrozole on normal breast epithelial cell proliferation and metabolic indices in postmenopausal women: a pilot study for breast cancer prevention. Cancer Epidemiol Biomarkers Prev 2002;11:614-621.

37. Donnellan PP, Douglas SL, Cameron DA, Leonard RC. Aromatase inhibitors and arthralgia. J Clin Oncol 2001;19:2767-2767.

38. Love RR, Newcomb PA, Wiebe DA, et al. Effects of tamoxifen therapy on lipid and lipoprotein levels in postmenopausal patients with node-negative breast cancer. J Natl Cancer Inst 1990;82:1327-1332.

39. Atalay G, Dirix L, Biganzoli L, et al. The effect of exemestane on serum lipid profile in postmenopausal women with metastatic breast cancer: a companion study to EORTC Trial 10951, 'randomized phase II study in first line hormonal treatment for metastatic breast cancer with exemestane or tamoxifen in postmenopausal patients.' Ann Oncol 2004;15:211-217. 


\section{Chapter 7}

Intergroup Exemestune Stuly:

results of the Endometrial sub protocol

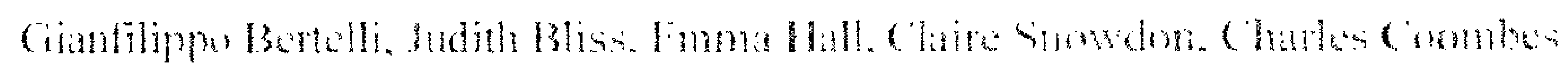

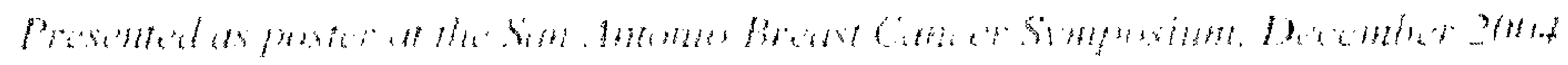




\section{Introduction}

The IES is an inter co-operative group, randomized, double-blind, phase 3 trial in postmenopausal women with early breast cancer. Patients disease free after 2-3 yrs tamoxifen were randomized to continue tamoxifen or switch to exemestane for a further 2-3 yrs to complete 5 yrs adjuvant endocrine therapy.

Tamoxifen induces uterine abnormalities detectable by ultrasound e.g. endometrial thickening, in postmenopausal women. The effects of switching to an anti-aromatase agent such as exemestane are not known, yet the IES study which reported earlier this year (NEJM 2004; 350: 1081-92) provides an ideal opportunity to evaluate this. Preliminary results of the IES Endometrial Sub-protocol, in which a subgroup of patients underwent detailed endometrial assessments, are reported.

\section{IES TRIAL DESIGN}

4740 patients with ER positive/unknown, histologically or cytologically confirmed, completely resected, adequately treated unilateral adenocarcinoma of the breast were randomized.

\section{ENDOMETRIAL. SUB-PROTOCOL DESIGN}

All patients from a subset of centers were considered.

Eligibility criteria included:

intact uterus

no previous endometrial cancer or other uterine malignancy

no undiagnosed vaginal bleeding within the past 12 months.

Primary endpoint:

proportion of patients with endometrial thickness $\geq 5 \mathrm{~mm}$ after 2 yrs of randomized treatment.

Secondary endpoints:

transvaginal ultrasound changes including mean endometrial thickness, mean uterine volume, presence of polyps, fibroids, ovarian cysts.

histological and immunohistochemical findings in patients who undergo biopsy or hysterectomy.

\section{Methods}

Transvaginal ultrasound examinations were performed at randomization and at 6,12 and 24 months to assess:

- Endometrial thickness (ET) (independently reviewed)

- Uterine volume

- Uterine diameter

- Other uterine findings

These evaluations were to be repeated 1 and 2 years after treatment completion. 


\section{Statistical considerations}

Planned sample size: 176 patients (200 allowing for a 15\% attrition rate) were required to detect a $25 \%$ difference in the proportion of patients with endometrial thickening (ET $\geq 5 \mathrm{~mm}$ ) (assuming 55\% in the tamoxifen arm and 30\% in the exemestane arm) with $90 \%$ power, $5 \%$ two-sided significance.

Statistical methods: The primary endpoint was analyzed using a chi-squared test. Within group changes in ET and uterine volume from baseline were analyzed as secondary endpoints using paired t-tests. Between group comparisons used two-sample t-tests and descriptive tables. All analyses used a $5 \%$ significance level and were performed by intention to treat.

Statistical power in this analysis: Following publication of main trial results, it was decided to analyse and release available data from each sub-protocol. At this time, the complete treated case subset of 90 patients provides $67 \%$ power.

\section{Patient population}

Two hundred and nineteen randomized patients were entered into the endometrial subprotocol. Analyses were conducted on the snapshot of data comprising all data received relating to visits to 30 th June 2003 . Two analysis subsets are considered here:

One hundred and eighty patients with endometrial assessments performed within windows of \pm 1 month of 6 and 12 month follow-ups, and \pm 2 months of 24 month followup. These assessment windows are in accordance with the protocol and provided 179 patients at baseline (1 patient-missing data on baseline ET), 137 at 6 mnths, 121 at 12 months and 96 at 24 months. This is an "all available data" analysis.

Ninety patients who had received at least 2 yrs randomized treatment and had an ontreatment ultrasound performed between 22 and 26 months from treatment start. This is a "treated and complete case" analysis.

Thirty six patients were entered from a single centre and have not been included in this analysis as it has not been possible to verify the reliability of data. Three patients were not included as they have no assessment data available.

\section{Results}

Baseline patient characteristics were well balanced between the treatment arms in both analysis populations (Table 1 ).

Most patients had not had endometrial ultrasound during pre-randomization tamoxifen treatment and of those who had, the majority was assessed as normal. Eleven/84 patients in the exemestane group and 14/96 in the tamoxifen group reported at least one abnormality. Twelve patients in each group had undergone investigations other than endometrial ultrasound. 
TABLE 1

Baseline patient and tumour characteristics.

\begin{tabular}{|c|c|c|c|c|c|}
\hline $\begin{array}{c}\text { Exemestane } \\
\mathrm{N}=84\end{array}$ & $\begin{array}{c}\text { Tamoxifen } \\
N=96\end{array}$ & & & $\begin{array}{c}\text { Exemestane } \\
\mathrm{N}=47\end{array}$ & $\begin{array}{c}\text { Tamoxifen } \\
\mathrm{N}=43\end{array}$ \\
\hline$\%$ & $\%$ & & & $\%$ & $\%$ \\
\hline 23.8 & 19.8 & & $<55$ & 23.4 & 14.0 \\
\hline 20.2 & 22.9 & & $55-59$ & 19.1 & 25.6 \\
\hline 22.6 & 18.8 & Age (years) & $60-64$ & 17.0 & 20.9 \\
\hline 16.7 & 25.0 & & $65-69$ & 19.1 & 30.2 \\
\hline 16.7 & 13.5 & & $70+$ & 21.3 & 9.3 \\
\hline 82.1 & 68.8 & & Positive & 80.9 & 67.4 \\
\hline 6.0 & 9.4 & ER Status & Negative & 8.5 & 14.0 \\
\hline 11.9 & 21.9 & & Unknown/Missing & 10.6 & 18.6 \\
\hline 41.7 & 44.8 & & Positive & 42.6 & 44.2 \\
\hline 16.7 & 12.5 & PgR Status & Negative & 17.0 & 11.6 \\
\hline 41.7 & 42.7 & & Unknown & 40.4 & 44.2 \\
\hline 75.0 & 66.7 & Suraerv Tyno & Mastectomy & 83.0 & 67.4 \\
\hline 25.0 & 33.3 & surgery tуре & BCT & 17.0 & 32.6 \\
\hline 16.7 & 11.5 & Use of HRT & Yes & 19.1 & 14.0 \\
\hline 48.8 & 52.1 & $\begin{array}{l}\text { Prior Use of } \\
\text { Chemotherapy }\end{array}$ & Yes & 44.7 & 39.5 \\
\hline
\end{tabular}

TABLE 2

Normalized ET at 24 Months (randomized patients within the visit windows).

\begin{tabular}{|c|c|c|c|c|c|}
\hline \multicolumn{2}{|c|}{} & \multicolumn{4}{|c|}{ Endometrial thickness at 24 month follow-up } \\
\cline { 3 - 6 } \multicolumn{2}{|c|}{} & \multicolumn{2}{|c|}{ Exemestane $(\mathrm{N}=84)$} & \multicolumn{2}{c|}{ Tamoxifen $(\mathrm{N}=96)$} \\
\cline { 2 - 6 } & $<5 \mathrm{~mm}$ & $\geq 5 \mathrm{~mm}$ & $<5 \mathrm{~mm}$ & $\geq 5 \mathrm{~mm}$ \\
\hline \multirow{2}{*}{$\begin{array}{c}\text { Endometrial } \\
\text { thickness at } \\
\text { baseline }\end{array}$} & $\geq 5 \mathrm{~mm}$ & $13(76.5 \%)$ & $4(23.5 \%)$ & $11(61.1 \%)$ & $7(38.9 \%)$ \\
\cline { 3 - 6 } & $\geq 5 \mathrm{~mm}$ & $15(48.4 \%)$ & $16(51.6 \%)$ & $6(20.0 \%)$ & $24(80.0 \%)$ \\
\hline
\end{tabular}

Figures la and $1 \mathrm{~b}$ show the proportion $(95 \% \mathrm{CI})$ of patients with endometrial thickness $\geq 5 \mathrm{~mm}$ at each assessment. Numbers of patients are also shown.

Thus, in randomized patients within the visit windows, the proportion with ET $>5 \mathrm{~mm}$ at the 24 month follow-up visit is $41.7 \%$ [95\% CI: $27.8 \%$ to $55.6 \%$ ] in the exemestane group and $64.6 \%$ [95\% CI: $51.5 \%$ to $77.7 \%$ ] in the tamoxifen group, $(p=0.024)$. Differences in the proportion with $\mathrm{ET} \geq 5 \mathrm{~mm}$ were also seen at 6 and 12 months $(p=0.008$ and 0.003 respectively). Similarly, for treated complete cases, the proportion with ET $\geq 5 \mathrm{~mm}$ at 24 months is $40.4 \%$ [ $95 \% \mathrm{CI}: 26.4 \%$ to $54.4 \%$ ] in the exemestane arm and $65.1 \%$ [95\% CI: $50.9 \%$ to $79.3 \%$ ] in the tamoxifen arm, $(\mathrm{p}=0.019)$.

In patients with an abnormal ET at baseline (i.e. $\geq 5 \mathrm{~mm}$ ), 15 patients in the exemestane arm $(48.4 \%)$ and 6 patients in the tamoxifen arm $(20.0 \%)$ had a normal ET (i.e. $<5 \mathrm{~mm}$ ) at 24 months (Table 2). A similar effect was also seen for treated complete cases, with 15 patients in the exemestane group $(50.0 \%)$ and 6 patients in the tamoxifen group $(21.4 \%)$ having a normal endometrial thickness after 2 years of treatment. 
FIGURE 1

a,b Percentage $(95 \% \mathrm{CI})$ of patients with $\mathrm{ET} \geq 5 \mathrm{~mm}$.

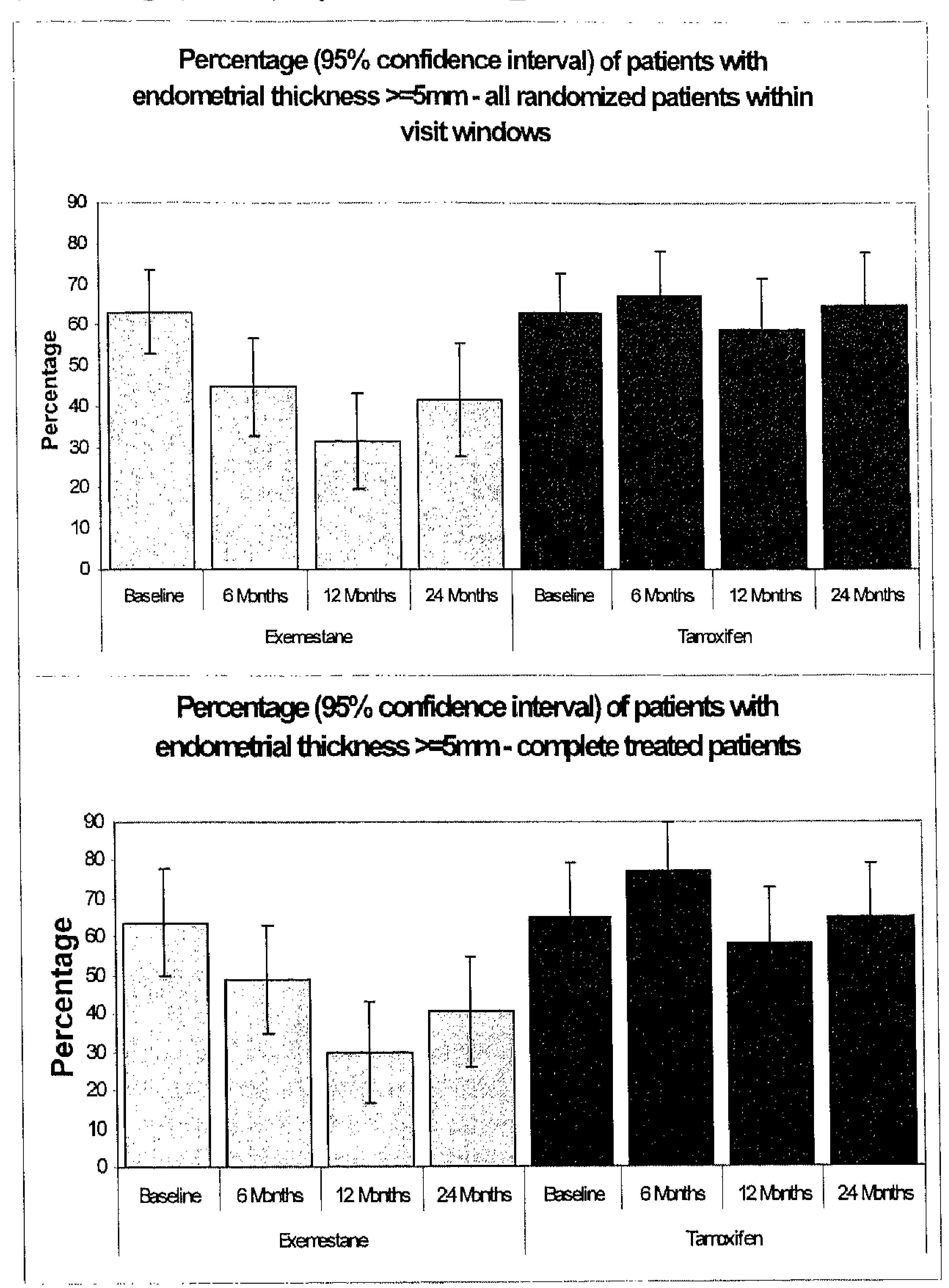

At 6 months, a significant change in ET was seen within the exemestane group (mean change $-1.75 \mathrm{~mm}, 95 \% \mathrm{CI}:-2.95$ to $-0.56, \mathrm{p}=0.005$ ) but not in the tamoxifen group (Table 3 and Figure 2). The comparison of mean change was significant at 6 months (mean difference $-1.70 \mathrm{~mm} ; 95 \% \mathrm{Cl}:-3.05$ to $-0.35, \mathrm{p}=0.014$ ). Mean uterine volume was also significantly reduced at 6 months in patients switching to exemestane (mean change $-15.5 \mathrm{~cm}^{3}, 95 \% \mathrm{Cl}:-23.40$ to $-7.51, \mathrm{p}=0.0002$ ) while remaining similar in the tamoxifen group (Table 4). 
FIGURE 2

a,b Mean change (95\% CI) in ET from baseline.

\section{All randomized patients in visit windows}

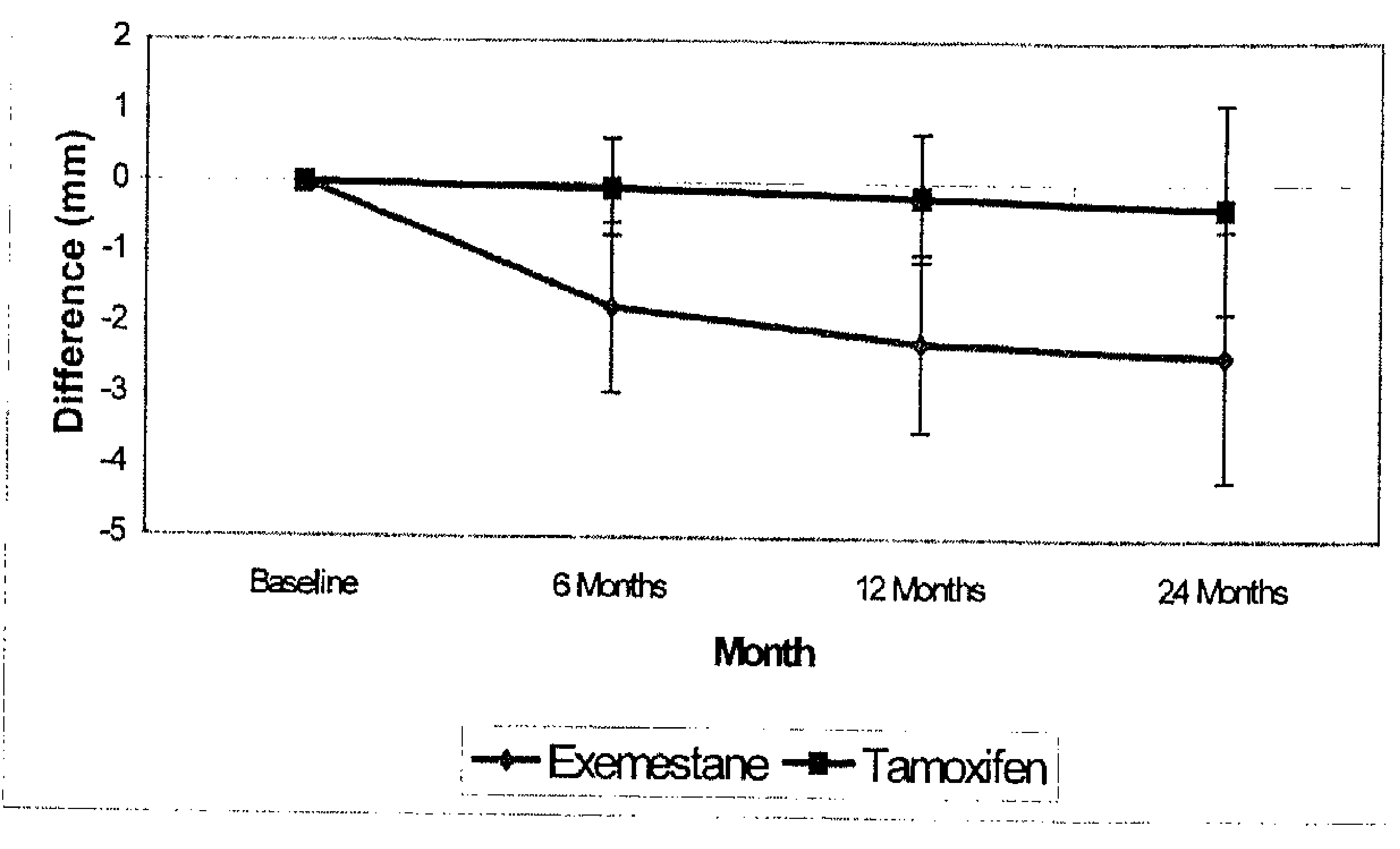

Treated complete cases

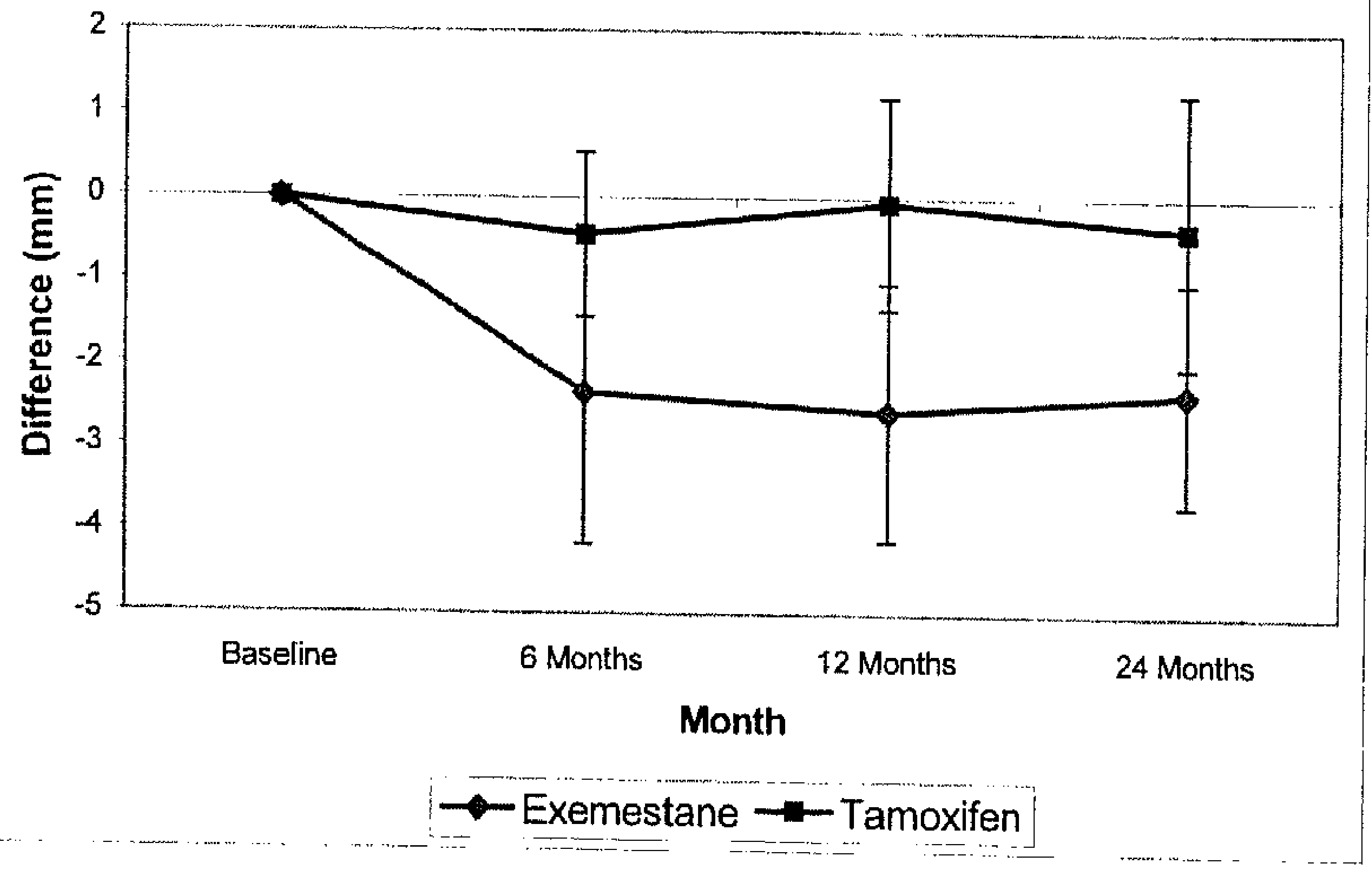


TABLE 3

Difference in ET $(\mathrm{mm})$ from baseline.

\begin{tabular}{|c|c|c|c|c|c|c|c|c|}
\hline & \multicolumn{4}{|c|}{ Exemestane } & \multicolumn{4}{|c|}{ Tamoxifen } \\
\hline & Baseline & $6 \mathrm{mth}$ & $12 \mathrm{mth}$ & $24 \mathrm{mth}$ & Baseline & $6 \mathrm{mth}$ & $12 \mathrm{mth}$ & $24 \mathrm{mth}$ \\
\hline $\begin{array}{l}\text { All available } \\
\text { data analysis }\end{array}$ & $N=84$ & $N=67$ & $N=60$ & $N=48$ & $N=95$ & $N=70$ & $N=61$ & $N=48$ \\
\hline $\begin{array}{l}\text { Mean } \\
\text { (SD) }\end{array}$ & $\begin{array}{r}7.24 \\
(5.49) \\
\end{array}$ & $\begin{array}{c}498 \\
(3.82)\end{array}$ & $\begin{array}{c}4.58 \\
(3.09)\end{array}$ & $\begin{array}{c}514 \\
(4.26)\end{array}$ & $\begin{array}{l}745 \\
(541)\end{array}$ & $\begin{array}{c}6.63 \\
(408)\end{array}$ & $\begin{array}{l}660 \\
(492)\end{array}$ & $\begin{array}{l}713 \\
(5.42)\end{array}$ \\
\hline $\begin{array}{l}\text { Mean change } \\
\text { from baseline } \\
\text { (SD) }\end{array}$ & - & $\begin{array}{c}-1.75^{\star \star} \\
(4.90)\end{array}$ & $\begin{array}{l}-2.24^{*} \\
(4.87)\end{array}$ & $\begin{array}{l}-242^{k *} \\
(6.06)\end{array}$ & - & $\begin{array}{l}-0.06 \\
(288)\end{array}$ & $\begin{array}{l}-0.20 \\
(351)\end{array}$ & $\begin{array}{l}-034 \\
(5.05)\end{array}$ \\
\hline $\begin{array}{l}\text { Complete case } \\
\text { analysis }\end{array}$ & & $n=47$ & $n=47$ & $n=47$ & & $n=40$ & $n=43$ & $n=43$ \\
\hline $\begin{array}{l}\text { Mean change } \\
\text { from baseline } \\
\text { (SD) }\end{array}$ & - & $\begin{array}{l}-2.35^{*} \\
(4.53)\end{array}$ & $\begin{array}{l}-2.57^{\text {*k }} \\
(5.28)\end{array}$ & $\begin{array}{c}-2.36^{* * *} \\
(613)\end{array}$ & - & $\begin{array}{l}.044 \\
(307)\end{array}$ & $\begin{array}{l}.006 \\
(4.23)\end{array}$ & $\begin{array}{l}.038 \\
(5.32)\end{array}$ \\
\hline
\end{tabular}

TABLE 4

Difference in Uterine Volume in $\mathrm{cm}^{3}$ from baseline.

\begin{tabular}{|c|c|c|c|c|c|c|c|c|}
\hline & \multicolumn{4}{|c|}{ Exemestane } & \multicolumn{4}{|c|}{ Tamoxifen } \\
\hline & Baseline & $6 \mathrm{mth}$ & $12 \mathrm{mth}$ & $24 \mathrm{mth}$ & Baseline & $6 \mathrm{mth}$ & $12 \mathrm{mth}$ & $24 \mathrm{mth}$ \\
\hline $\begin{array}{l}\text { All available } \\
\text { data analysis }\end{array}$ & $N=79$ & $N=65$ & $N=58$ & $N=42$ & $N=92$ & $N=69$ & $N=58$ & $N=51$ \\
\hline $\begin{array}{l}\text { Mean } \\
\text { (SD) } \\
\end{array}$ & $\begin{array}{c}48.0 \\
(36.0)\end{array}$ & $\begin{array}{c}32.0 \\
(25.1)\end{array}$ & $\begin{array}{c}27.9 \\
(16.3) \\
\end{array}$ & $\begin{array}{c}28.7 \\
(16.0)\end{array}$ & $\begin{array}{c}49.6 \\
(26.9) \\
\end{array}$ & $\begin{array}{r}45.8 \\
(28.8) \\
\end{array}$ & $\begin{array}{c}46.3 \\
(363) \\
\end{array}$ & $\begin{array}{c}50.2 \\
(34.4) \\
\end{array}$ \\
\hline $\begin{array}{l}\text { Mean change } \\
\text { from baseline } \\
\text { (SD) }\end{array}$ & - & $\begin{array}{l}-15.5^{*} \\
(32.1)\end{array}$ & $\begin{array}{l}-22.9^{*} \\
(36.6)\end{array}$ & $\begin{array}{l}-20.7^{*} \\
(326)\end{array}$ & - & $\begin{array}{c}-1.4 \\
(18.1)\end{array}$ & $\begin{array}{c}-0.1 \\
(20.2)\end{array}$ & $\begin{array}{c}1.2 \\
(23.0)\end{array}$ \\
\hline $\begin{array}{l}\text { Complete case } \\
\text { analysis }\end{array}$ & & $n=44$ & $n=42$ & $n=40$ & & $n=38$ & $n=40$ & $n=43$ \\
\hline $\begin{array}{l}\text { Mean change } \\
\text { from baseline } \\
\text { (SD) }\end{array}$ & - & $\begin{array}{l}-17.8 * \\
(30.3)\end{array}$ & $\begin{array}{l}-23.0^{*} \\
(31.6)\end{array}$ & $\begin{array}{l}-20.4^{*} \\
(32.9)\end{array}$ & - & $\begin{array}{c}0.6 \\
(17.6)\end{array}$ & $\begin{array}{c}2.7 \\
(20.7)\end{array}$ & $\begin{array}{c}2.0 \\
(23.6)\end{array}$ \\
\hline
\end{tabular}

\section{Conclusions}

After two years of randomized treatment, the proportion of patients with ET $\geq 5 \mathrm{~mm}$ was significantly reduced in patients switching to exemestane.

A significant reduction in ET and uterine volume was observed at 6 months in the exemestane group, and although the thickness continued to decrease, no further significant changes were observed. Switching to exemestane allows reversal of sub-clinical uterine abnormalities associated with tamoxifen.

Further follow-up will allow a more complete analysis of endometrial changes whilst on treatment (to 24 months). Information on post-treatment changes is still required. Assessments at 1 and 2 years after completion of randomized treatment will elucidate this. 


\title{
Chapter 8
}

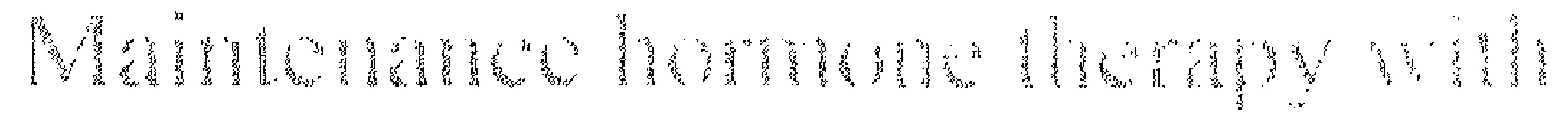

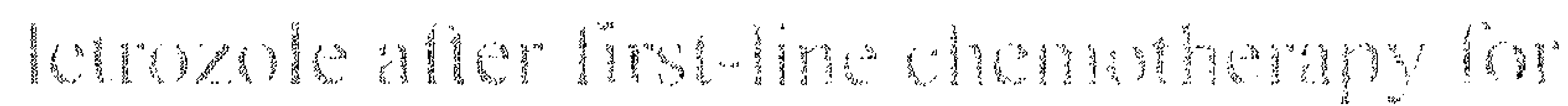 \\ udvanced hreast concri
}

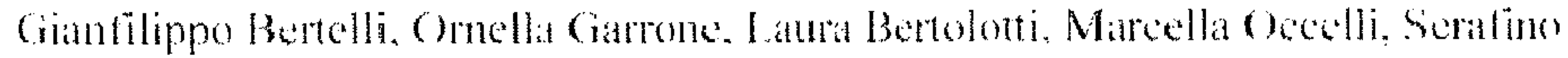
Conforti, Nicola Marzano. Antonio Pebbraro. Paolo Carlini, Christina Liossi. Luciat Del Mastro, and Robert C.E. L comard

Oncologe $2005 ; 6 x ; 36+2-370$ 


\section{Summary}

Objectives. Maintenance hormone therapy, after first-line chemotherapy, is routinely used by many clinicians in advanced breast cancer patients with potentially hormone-sensitive tumours. There is, however, insufficient evidence in the literature to support this practice. We investigated the effects of the third-generation aromatase inhibitor letrozole as a maintenance therapy in postmenopausal patients who had responded or had stable disease with first-line chemotherapy.

Methods. Fifty-eight patients (median age 62 years, range 31-80) were recruited and received letrozole, 2.5 mg/day starting within 8 weeks since the last cycle of chemotherapy. Estrogen and/or progesterone receptor status was positive in $81 \%$ of the patients, unknown in $19 \% ; 57 \%$ of the patients had visceral diseasc. Firstline chemotherapy included anthracyclines and/or taxanes in $74 \%$ of cases.

Results. The median time to progression from starting letrozole was 18.5 months. A shorter time to progression was found in patients with abnormal CA 15-3 levels at the start of maintenance letrozole (median TTP, 9.9 months: $p=.01$ ), or with levels increasing $>25 \%$ from baseline during the first six months of letrozole therapy (median TTP, 8.2 months: p<.0001). Response status improved during letrozole in $15.5 \%$ of patients who had obtained less than a complete response to chemotherapy. Maintenance treatment was well tolerated and had no significant impact on quality of lile scores.

Conclusions. This study provides evidence in support of the common clinical practice of maintenance hormone therapy after chemotherapy in suitably selected patients with advanced breast cancer.

\section{Introduction}

The optimal duration of cytotoxic treatment for metastatic breast cancer is controversial. Several trials have explored the benefit of extending chemotherapy duration in patients whose disease responds or stabilizes, showing that maintenance treatment can prolong response duration and time to progression, although survival does not seem to be improved [1-3]. In a recent EORTC study, non-progressing patients after six courses of CMF were randomised to stop or continue treatment: continuation of CMF resulted in a significantly longer time to treatment failure ( 5.2 vs. 3.5 months), without however any gain in overall survival or in quality-adjusted survival time (Q-TWiST) [4]. In the absence of clear evidences of benefit from prolonged chemotherapy, many clinicians prefer to interrupt the treatment after six-eight cycles, and observe the patient without further treatment until progression.

An attractive alternative to watchful waiting, at least in potentially endocrine-responsive patients (those with hormone receptor-positive tumours, or unknown receptor status), is administration of maintenance hormone therapy. As opposed to maintenance chemotherapy, one would at least expect that any therapeutic gain will be obtained with relatively little toxicity, thus with a potential net benefit for quality of life. This strategy, however, has not been adequately explored so far. Only one prospective study has been published [5], where medroxyprogesterone acetate was administered as maintenance therapy after first-line chemotherapy in a group of patients, which included premenopausal $(48 \%)$ and estrogen receptor-negative $(39 \%)$ women. Despite these limitations, the study showed a significant, albeit modest improvement of time to progression (TTP), from 3.7 months in the control group to 4.9 months in the medroxyprogesterone acetate group. Medroxyprogesterone acetate, however, is a progestational agent, which can have side effects in a substantial proportion of patients, and indeed, $15 \%$ of the patients in the above trial discontinued treatment because of toxicity (weight gain, oedema, pulmonary embolism). 
With the advent of new, more powerful and tolerable endocrine agents in recent years [6], we felt that the concept of maintenance hormone therapy after chemotherapy was worth exploring again. Letrozole is a third-generation aromatase inhibitor, which has shown superior efficacy and tolerability to progestins and superior efficacy to tamoxifen $[7,8]$. In this article, we report the results of the MANTLE (Maintenance Therapy with Letrozole) trial, an exploratory study of maintenance hormone therapy in postmenopausal, hormone-responsive breast cancer patients who have responded or stabilized to first-line chemotherapy for advanced disease.

\section{Patients and Methods}

Eligibility criteria. Eligible patients had histologically or cytologically documented breast cancer, with measurable or evaluable metastatic disease that had responded or stabilized to first-line chemotherapy. Any single-agent or polychemotherapy regimen for breast cancer was allowed as first-line treatment, provided that the duration of chemotherapy had been at least 3 months, that the patient had not progressed, and that no more than 8 weeks had elapsed since the administration of the last cycle. Previous adjuvant chemotherapy and/or adjuvant tamoxifen were allowed. Previous treatment with tamoxifen for palliation of metastatic disease, before first-line chemotherapy, was allowed. Previous radiotherapy (adjuvant or palliative) was allowed, provided that the patient had at least one non-irradiated site of measurable or evaluable disease. Patients already receiving bisphosphonates were allowed to continue them and could be entered into the trial, provided there were evaluable sites of disease other than bone. Patients had to be postmenopausal, as defined by one of the following: absence of menses for at least 12 months, or age $>50$ years and previous hysterectomy, or previous surgical or radiation oophorectomy, or amenhorreic status induced by LHRH-analogues. Positivity of estrogen receptors (ER) and/or progesterone receptors (PgR), or unknown tumour receptor status, was also required. Positivity of hormone receptors was defined as $\geq 10$ fmol/mg/cytosol protein when assessed by dextran-coated charcoal method, or $\geq 10 \%$ positive cells by immunohistochemistry method. Additional eligibility criteria were: World health Organization (WHO) performance status (PS) $\leq 3$, recovery from toxicity of previous chemotherapy, written informed consent. The study was approved by the Ethical Committee of the National Cancer Institute in Genoa and by participant centres' local ECs, according to national and institutional guidelines. Exclusion criteria were: male sex, previous treatment with aromatase inhibitors or any other endocrine agent other than tamoxifen, life expectancy less than 3 months, severe psychiatric conditions preventing informed consent and/or follow up.

Treatment. All patients received letrozole, $2.5 \mathrm{mg}$ daily p.o., starting within eight weeks from the last cycle of chemotherapy. The administration of letrozole continued until tumour progression or withdrawal due to toxicity or patient's decision.

Evaluations. The primary endpoint of the study was to evaluate time to progression. Secondary endpoints were the assessment of changes in CA 15-3 levels, response status, performance status, and quality of life during maintenance treatment with letrozole. Time to progression was defined as the time from starting letrozole to progression, or cancer-related death, or death form unknown cause during therapy, or the last day of follow up for patients who had not progressed at the date of analysis. 
Patients were subjected to the following assessments: tumor assessment (WHO criteria), performance status (PS, WHO criteria), EORTC QLQ-C30 questionnaire [9], and CA15-3 serum level. Those assessments were made at baseline and subsequently every three months. Patients were assessed to detect an improvement of objective tumor response and/or PS. CA15-3 was measured by each participant center's local laboratory; normal values for each laboratory were obtained for normalization.

Statistical methods. All analyses are considered as exploratory in nature as the study was not comparative. Time to progression was analysed by means of Kaplan-Meier procedure and Cox proportional hazards model. Backward elimination process was adopted to select variables giving important contribution to the model (a threshold of 0.05 was set to retain a variable in the model). No protection for multiple comparisons was applied to log-rank test results when comparing couples of strata. Descriptive statistics were used for presenting other variables.

As no expected proportions were available for comparing proportion of patients reaching a clinically important target with present data, confidence intervals $(\mathrm{CI})$ were adopted to evaluate results. Exact method was chosen assuming binomial distribution. Confidence level was set at $95 \%$. Change and percentage change from baseline of quality of life total score were tested by means of Student $t$ test for paired samples.

\section{Results}

Fifty-eight patients were recruited into the study by 21 Italian centres between August 1999 and December 2001. Table 1 summarizes patients' characteristics at study entry. Participating patients had received chemotherapy regimens consistent with current standards for first-line therapy [10]: treatment included anthracyclines and/or taxanes in $74.1 \%$ of cases, while CMF or other (mainly vinorelbine-based) regimes were used in the remaining $25.9 \%$. Adjuvant chemotherapy had been received by $63.8 \%$ of patients, and tamoxifen - as adjuvant therapy or for advanced disease - by $77.6 \%$. Most patients $(56.9 \%)$ had visceral disease, consistent with the fact that chemotherapy was chosen as their first-line therapy. Thirteen patients $(22.4 \%)$ were receiving bisphosphonates at entry and continued them.

Time to progression. At the time of analysis, 34 patients $(58.6 \%)$ had interrupted letrozole because of disease progression, while $24(41.4 \%)$ were still on treatment without progression. The median time to progression (TTP) in the whole group is 18.5 months ( $95 \% \mathrm{CI}=9.9$ to 30.8 months) (Figure 1). Only six patients have died $(10.3 \%)$. The mean survival time, estimated by means of Kaplan-Meier procedure, was 12 months (median not reached).

Changes in Ca 15-3 levels. Baseline (at study entry) CA 15-3 values were available for 54 patients: these were above the upper limit of normal (ULN) for the local laboratory in $29(53.7 \%)$. Time to progression appeared longer in patients with normal vs. abnormal CA $15-3$ at baseline ( 30.8 months vs. 9.9 months, $p=0.010$ log-rank test). Trends in C.A15-3 levels between baseline and visit 2 (approximately six month after starting letrozole) were also analysed, after excluding 16 patients who did not provide $\mathrm{Ca}$ 15-3 values during this period or progressed before visit 2. Based on the marker's behaviour, three groups of patients were identified. Patients who obtained at least a $25 \%$ decrease of initially abnormal $\mathrm{Ca} 15-3$ levels $(n=8)$ had prolonged benefit from maintenance 
TABLE 1

Patients' characteristics.

\begin{tabular}{|c|c|}
\hline \multicolumn{2}{|l|}{ Age (years): } \\
\hline Median (range) & $62(31-80)$ \\
\hline \multicolumn{2}{|l|}{ Disease-free interval (months): } \\
\hline \multirow[t]{2}{*}{ Mcdian (range) } & $37(0-180)$ \\
\hline & No. of patients $(\%)$ \\
\hline \multicolumn{2}{|l|}{ Nodal status at diagnosis: } \\
\hline Unknown & $4(6.9)$ \\
\hline Node negative & $21(36.2)$ \\
\hline Node positive & $33(56.9)$ \\
\hline \multicolumn{2}{|l|}{ Receptor status: } \\
\hline Both unknown & $11(19)$ \\
\hline $\mathrm{ER}+\mathrm{PgR}+$ & $36(62.1)$ \\
\hline $\mathrm{ER}+\mathrm{PgR}-$ & $7(12.1)$ \\
\hline $\mathrm{ER}-\mathrm{PgR}+$ & $1(1.7)$ \\
\hline \multicolumn{2}{|l|}{ Adjuvant chemotherapy: } \\
\hline None & $21(36.2)$ \\
\hline $\mathrm{CMF}$ & $18(3 !)$ \\
\hline Anthracycline based & $15(25.9)$ \\
\hline Taxane based & $4(6.9)$ \\
\hline \multicolumn{2}{|l|}{ Previous Tamoxifen: } \\
\hline No & $13(22.4)$ \\
\hline Adjuvant & $39(67.2)$ \\
\hline For advanced disease & $6(10.3)$ \\
\hline \multicolumn{2}{|l|}{ First-line chemotherapy: } \\
\hline $\mathrm{CMF}$ & $6(10.3)$ \\
\hline Anthracycline based & $17(29.3)$ \\
\hline Taxane based & $11(19)$ \\
\hline Anthracycline + Taxanes & $15(25.9)$ \\
\hline Other & $9(15.5)$ \\
\hline \multicolumn{2}{|c|}{ Response to first line chemotherapy: } \\
\hline $\mathrm{CR}$ & $13(22.4)$ \\
\hline PR & $36(44.8)$ \\
\hline $\mathrm{SD}$ & $19(32.8)$ \\
\hline \multicolumn{2}{|l|}{ Dominant metastatic site: } \\
\hline Viscera & $33(56.9)$ \\
\hline Bones & $20(34.5)$ \\
\hline Soft tissues & $5(8.6)$ \\
\hline \multicolumn{2}{|l|}{ Performance status: } \\
\hline 0 & $31(53.4)$ \\
\hline 1 & $18(31.0)$ \\
\hline 2 & $8(13.8)$ \\
\hline 3 & $1(1.7)$ \\
\hline
\end{tabular}


FIGURE 1

Time to progression from the start of maintenance therapy with letrozole, in the whole group of patients $(\mathrm{n}=58)$.

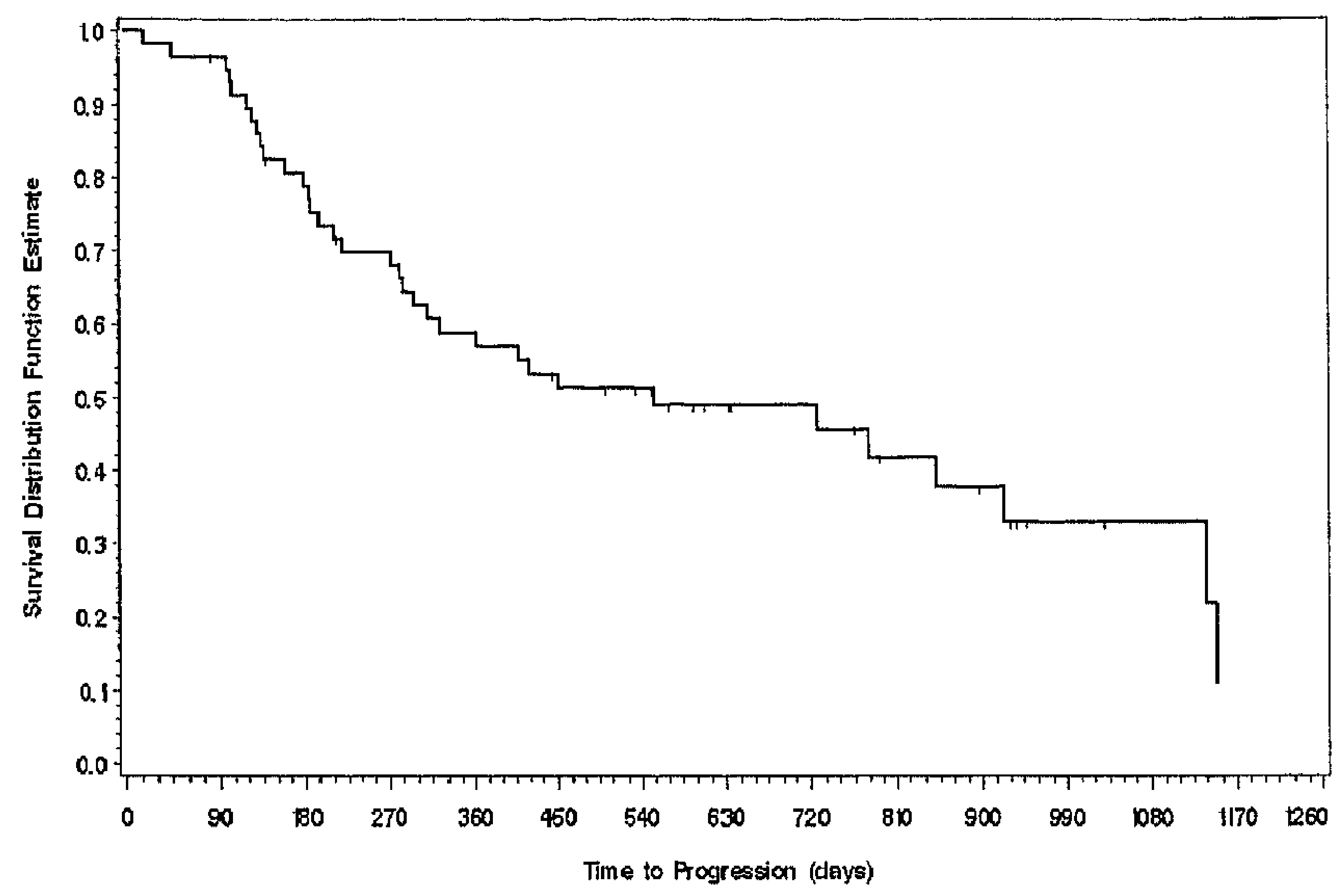

FIGURE 2

Time to progression by $\mathrm{Ca} 15-3$ trend during the first two visits.

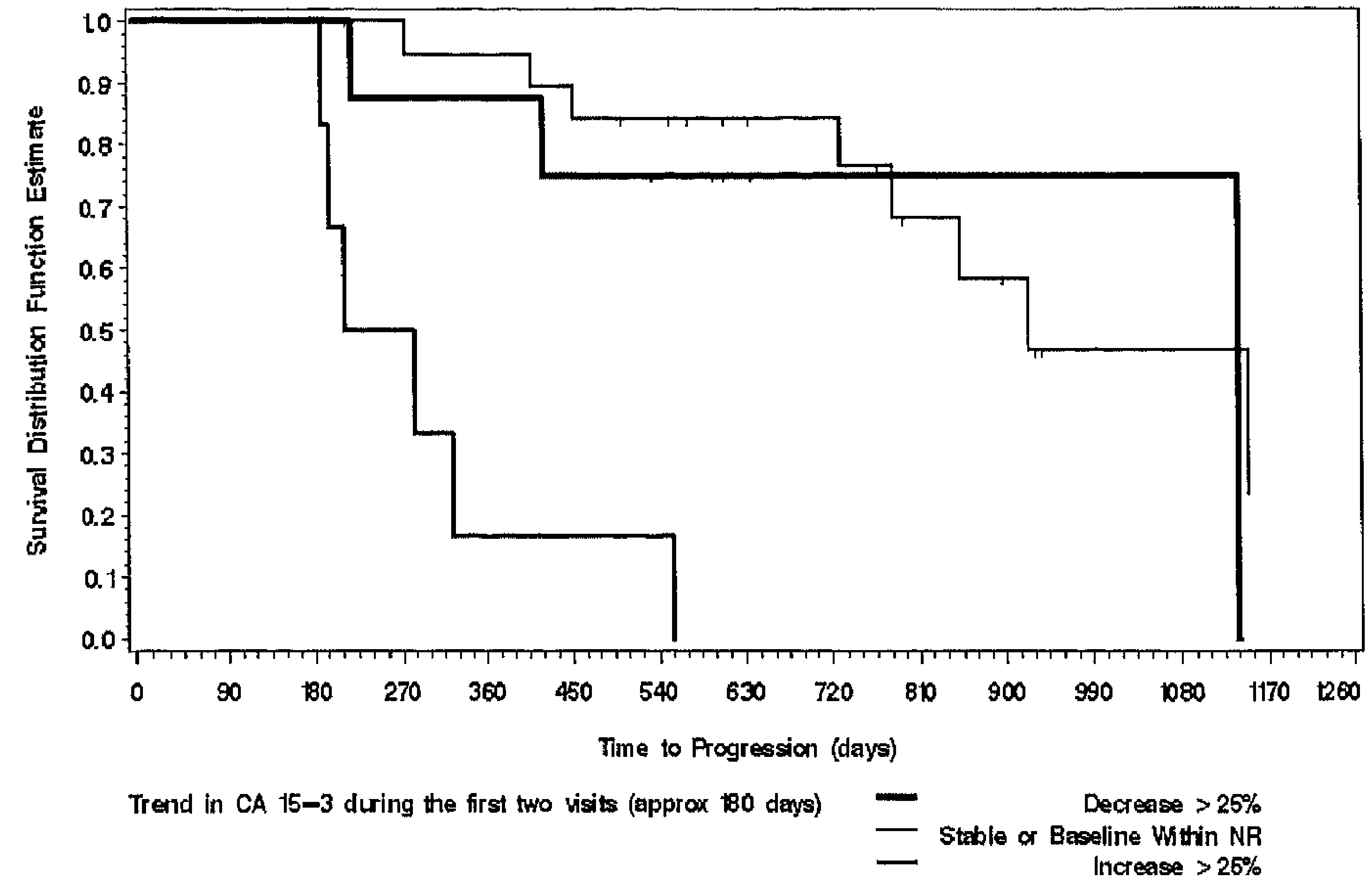


treatment, with a median TTP of 38 months. Median baseline values of CA 15-3 in this group were $2.79 \times$ ULN, which was reduced to $1.8 \times$ ULN at visit $1,1.38 \times$ ULN at visit 2 , and $1 \times U L N$ at visit 5. Patients $(n=24)$ with normal or elevated CA $15-3$ at baseline, who had less than $25 \%$ variations in the marker's level during the first 2 visits, also appeared to have a long TTP (median 30.8 months). Conversely, an increase of $\geq 25 \%$ of the marker during the first six months $(n=6)$ seemed predictive of more rapid disease progression (median TTP 8.2 months). Differences in TTP were statistically significant for the group with increasing marker levels vs. the group with stable or within normal levels ( $<<0.0001, \log$-rank test), and not significant for the group with decreasing levels vs. the group with stable or within normal levels ( $\mathrm{p}=0.53$ : Figure 2 ).

Changes in quality of life. Responses to the EORTC QLQ C-30 questionnaires were linearly transformed to give scores from 0 to 100 . Incomplete questionnaires were handled according to EORTC's recommendations: when at least half the items in a scale were present, missing values were recorded as their mean. There were no statistically significant variations in mean global health status scores during the study. The subscales for physical, emotional, cognitive, role and social functioning also did not show significant variations from baseline by means of the paired-samples t-test, as well as the symptoms subscales for pain, fatigue, nausea and vomiting, diarrhoea, constipation, insomnia, appetite loss, and dyspnoea.

Side effects. Tolerability was excellent. No patient interrupted letrozole for reasons other than progression. No grade 3-4 toxicity was reported. Grade 1-2 toxicities included asthenia in 4 patients $(6.9 \%)$, hot flushes in $2(3.4 \%)$, headache in $2(3.4 \%)$, gastrointestinal complaints and arthralgia in 1 patient respectively $(1.7 \%)$.

Factors correlated with TTP. Univariate analysis of TTP based on nodal and receptor status at diagnosis, exposure to adjuvant chemotherapy, disease-free interval, type of first-line chemotherapy, response to first line chemotherapy, and dominant site of metastases were performed. Only nodal status and response to first line chemotherapy significantly correlated with TTP $(\mathrm{p}=0.035$ for complete response vs. partial response or stability, log-rank test). A Cox model was set up, which included the above variables plus CA 15-3 (abnormal vs. normal at baseline; 54 patients with valid data). The backward elimination process left only "CA 15-3 abnormal at baseline" as a significant variable $(\mathrm{P}=0.013$ and Hazard Ratio=2.6). A second model was set up, which included also CA 15-3 trend (38 patients with valid data). The backward elimination process left only "CA 15-3 trend" as significant variable $(\mathrm{p}<0.0001$ and Hazard Ratio $=13.0$ for the contrast "increasing vs. stable or normal at baseline"). The contrast "decreasing vs. stable or normal at baseline" was tested and not retained in the final model $(\mathrm{p}=0.122)$.

\section{Discussion}

Despite its widespread use in routine clinical practice, there is a lack of systematic data on the impact of endocrine therapy used as maintenance therapy for hormone receptorpositive advanced breast cancer following chemotherapy. This is almost certainly because maintenance hormone therapy is presumed to be useful and well tolerated. However, without a quantification of these effects, this clinical practice is hardly defensible as evidence-based. The only prospective study available so far was conducted with medroxyprogesterone acetate in a mixed population of ER-positive and ER-negative 
patients [5]; two other studies have suggested benefit from maintenance hormone therapy after conventional [11] and high-dose chemotherapy [12], respectively, but their retrospective nature does not allow firm conclusions.

The present study has quantified the benefits of a well tolerated and potent aromatase inhibitor used as maintenance treatment after chemotherapy, and has demonstrated a progression-free survival of more than 18 months for the whole group. Since the study was not randomised, it is not possible to affirm conclusively that letrozole contributed to the prolonged time to progression in our patients, which however compares favourably with the median time to progression of 7.8 months reported in the observation arm of a randomised ECOG (Eastern Cooperative Oncology Group) trial of maintenance therapy following complete remission to induction chemotherapy [13]. Patients randomised to receive maintenance treatment in this trial obtained a median time to progression of 18.7 months: interestingly, the maintenance regimen (CMFPTH: cyclophosphamide, methotrexate, fluorouracil, prednisone, tamoxifen and halotestin) included endocrine manipulation, and $74 \%$ of the patients had ER-positive or ER-unknown disease.

Some insights may also be gained by examination of the tumour marker profile in our study. There were cases where a sharp fall in the Ca 15.3 level occurred early after commencing letrozole. These patients, regardless of objective clinical response, also had a prolonged time to disease progression. A similar observation was made concerning those patients who commenced letrozole with a normal level of marker and sustained that marker level during the progression-free period. On the other hand, patients with an early increase in CA 15-3 levels progressed rapidly. The use of tumour markers, such as CA 15-3, to monitor patients in this setting is supported by recent findings of a study where markers' sensitivity for progression was $68 \%$ in 112 patients after first-line chemotherapy, with a lead-time of more than 3 months in $27 \%$ of cases [14].

The overall results of our study do seem to justify the common clinical practice of maintenance hormone therapy after chemotherapy in ER-positive patients with advanced breast cancer. One of the major benefits of this study should be to encourage this sort of research in a group of patients where we can be absolutely sure that relapse will occur. The potential of low-toxicity interventions as maintenance therapy in hormoneinsensitive cancers, where the natural history of the disease is probably more aggressive and where the impact of the intervention is even less certain, should be investigated. Very low dose cytotoxic agents, e.g. cyclophosphamide and methotrexate, which appear to be effective in metastatic breast cancer, could act as angiogenesis inhibitors in this setting. New specific anti-angiogenesis agents under development could also be tested. Vaccine trials have already been reported, with the endpoint of relapse free survival being a consistent and reliable one [15]. The observations on CA 15-3 emerged from our trial also suggest areas for further study. Early, low-toxicity interventions based on marker behaviour could produce improved time to symptomatic relapse: proteomic research has a potential to yield more useful information in this area, reflecting tumour biology better than traditional tumour markers. 


\section{References}

1. Coates A, Gebski V, Bishop JF, Jeal PN, Woods RL, Snyder R, Tattersall MH, Byrne M, Harvey V, Gill G. Improving the quality of life during chemotherapy for advanced breast cancer. A comparison of intermittent and continuous treatment strategies. $\mathrm{N}$ Engl J Med 1987;317:1490-5.

2. Muss HB, Case LD, Richards F 2nd, White DR, Cooper MR, Cruz JM, Powell BL, Spurr $\mathrm{CL}$, Capizzi RL. Interrupted versus continuous chemotherapy in patients with metastatic breast cancer. The Piedmont Oncology Association. N Engl J Med 199;325:1342-8.

3. Gregory RK, Powles TJ, Chang JC, Ashley S. A randomised trial of six versus twelve courses of chemotherapy in metastatic carcinoma of the breast. Eur J Cancer 1997;33:2194-7.

4. Nooij MA, de Haes JC, Beex LV, Wildiers J, Klijn J, Becquart D, Jassem J, Engelsman E, Duchateau L; EORTC Breast Cancer Group. Continuing chemotherapy or not after the induction treatment in advanced breast cancer patients. clinical outcomes and oncologists' preferences. Eur J Cancer 2003;39:614-21.

5. Kloke O, Klaassen U, Oberhoff C, Hartwich G, Szanto J, Wolf E, Heckmann M, Huhn R, Stephan L, Schnepper U, Donsbach GM, Bechtel C, Rudolph R, Berke A, Borquez D, Hawig I, Hirche H, Schindler AE, Seeber S, Becher R. Maintenance treatment with medroxyprogesterone acetate in patients with advanced breast cancer responding to chemotherapy: results of a randomized trial. Essen Breast Cancer Study Group. Breast Cancer Res Treat 1999;55:51-9.

6. Smith IE, Dowsett M. Aromatase inhibitors in breast cancer. N Engl J Med 2003;348:2431-42.

7. Mouridsen H, Gershanovich M, Sun Y, Perez-Carrion R, Boni C, Monnier A, Apffelstaedt J, Smith R, Sleeboom HP, Janicke F, Pluzanska A, Dank M, Becquart D, Bapsy PP, Salminen E, Snyder R, Lassus M, Verbeek JA, Staffler B, Chaudri-Ross HA, Dugan M. Superior efficacy of letrozole versus tamoxifen as first-line therapy for postmenopausal women with advanced breast cancer: results of a phase III study of the International Letrozole Breast Cancer Group. J Clin Oncol 2001;19:2596-606.

8. Dombernowsky P, Smith I, Falkson G, Leonard R, Panasci L, Bellmunt J, Bezwoda W, Gardin G, Gudgeon A, Morgan M, Fornasiero A, Hoffmann W, Michel J, Hatschek T, Tjabbes T, Chaudri HA, Hornberger U, Trunet PF. Letrozole, a new oral aromatase inhibitor for advanced breast cancer: double-blind randomized trial showing a dose effect and improved efficacy and tolerability compared with megestrol acetate. J Clin Oncol 1998;16:453 -61.

9. Aaronson NK, Ahmedzai S, Bergman B, Bullinger M, Cull A, Duez NJ, Filiberti A, Flechtner $\mathrm{H}$, Fleishman SB, de Haes JC, ct al. The European Organization for Research and Treatment of Cancer QLQ-C30: a quality-of-life instrument for use in international clinical trials in oncology. J Natl Cancer Inst 1993;85:365-76.

10. Cazzaniga M, Pronzato P, Leto di Priolo SL, De Matteis A, Di Costanzo F, Passalacqua R, Rosso R, Torri V. Patterns of relapse and modalities of treatment of breast cancer: the 'IRIS' Project, a multicenter observational study. Oncology 2004;66:260-8.

11. Berruti A, Zola P, Buniva T, Bau MG, Farris A, Sarobba MG, Bottini A, Tampellini M, Durando A, Destefanis M, Monzeglio C, Moro G, Sussio M, Perroni D, Dogliotti L. Prognostic factors in metastatic breast cancer patients obtaining objective response or disease stabilization alter first-line chemotherapy with epirubicin. Evidence for a positive effect of maintenance hormonal therapy on overall survival. Anticancer Res 1997;17:2763-8. 
12. Montemurro F, Rondon G, Ueno NT, Munsell M, Gajewski JL, Champlin RE. Factors affecting progression-free survival in hormone-dependent metastatic breast cancer patients receiving high-dose chemotherapy and hematopoietic progenitor cell transplantation: role of maintenance endocrine therapy. Bone Marrow Transplant 2002;29:861-6.

13. Falkson G, Gelman RS, Pandya KJ, Osborne CK, Tormey D, Cummings FJ, Sledge GW, Abeloff MD. Eastern Cooperative Oncology Group randomized trials of observation versus maintenance therapy for patients with metastatic breast cancer in complete remission following induction treatment. J Clin Oncol 1998;16:1669-76.

14. Soletormos G, Nielsen D, Schioler V, Mouridsen H, Dombernowsky P. Monitoring different stages of breast cancer using tumour markers CA 15-3, CEA and TPA. Eur I Cancer 2004;40:481-6.

15. Miles D, Ibrahim N, Roché H, Guillem V, Martin M, Perren T, Cameron D, Glaspy J, Dodwell D, Parker J, Tres A. An international, randomized phase III clinical trial of STn- KLH (Theratope $(\mathbb{B})$ therapeutic cancer vaccine in metastatic breast cancer patients. San Antonio Breast Cancer Symposium, December 3-6 2003 (abs.n. 36). 


\section{Chapter 9}

\section{Sequential treatment with exemestane and non-steroidal aromatase inhibitors in advanced breast cancer}

Gianfilippo Bertelli, Onella Carrone, Mareo Merlano. Marcella Occelli, Laura Bertoloti, lederico Castiglione, Forella Pepi, Onella Fusco. L.ucia Del Mastro and Robert C.F.Lemard

Oncology $2006: 69: 471-47 ?$ 


\section{Summary}

Background. The steroidal aromatase inactivator exemestane has demonstrated activity after prior failure of non-steroidal aromatase inhibitors (including third-generation inhibitors letrozole and anastrozole) in postmenopausal women with advanced breast cancer. If exemestane is used as first anti-aromatase agent, however, it is unclear whether patients can still benefit from letrozole or anastrozole after progression.

Patients and methods. Postmenopausal patients with advanced, hormone receptor-positive or -unknown breast cancer were eligible for this study. Patients with no prior exposure to anti-aromatase drugs received exemestane. $25 \mathrm{mg}$ daily, as first anti-aromatase agent. At the time of progression, patients were crossed-over to anastrozole or letrozole if further endocrine therapy was considered appropriate. Patients with prior exposure to anti-aromatase agents were also included in the study, and were given anastrozole or letrozole if they had previously received exemestane, or exemestane if they had previously received anastrozole or letrozole. The primary endpoint of the study was the clinical benefit (C.B) rate (complete response + partial response + stabilization of disease for $\geq 24$ weeks).

Results. Forty patients reccived exemestane $25 \mathrm{mg}$ daily as first anti-aromatase agent, with a CB rate of $67.5 \%(95 \%$ C.I., $52.9 \%-82.0 \%)$ and a median time to progression (TTP) of 9.6 months. In 18 patients, letrozole $(n=17)$ or anastrozole $(n=1)$ were used after failure of exemestane: the C.B rate was $55.6 \%$ (95\% C.I., $32.6 \%-78.5 \%$ ) with a median TTP of 9.3 months. In 23 patients, exemestane was used after failure of letrozole or anastrozole: the CB rate was $43.5 \%(95 \%$ C.I., $23.2 \%-63.7 \%)$ with a median TTP of 5.1 months.

Conclusions. Our study confirms that exemestane is active after prior failure of letrozole or anastrozole. We have also shown that patients can receive exemestane as their first anti-aromatase agent, and still benelit from letrozole or anastrozole after progression. This suggests that the partial non-cross resistance between steroidal and non-steroidal anti-aromatase agents is independent of the sequence employed.

\section{Introduction}

Anti-aromatase treatment is rapidly gaining acceptance as first-choice endocrine manipulation in postmenopausal women with hormone-sensitive breast cancer. Comparative trials against tamoxifen support the use of third-generation anti-aromatase agents anastrozole, letrozole and exemestane in the setting of first-line endocrine therapy for metastatic disease [1-4] or as neoadjuvant therapy [5-7]. All three agents are also currently being tested in the adjuvant setting in large multinational trials, with preliminary results consistently showing a significant improvement of disease free survival [8-11]. In January 2005, The American Society of Clinical Oncology updated its guidelines on adjuvant therapy for postmenopausal women with hormone receptor-positive breast cancer [12], recommending the inclusion of an anti-aromatase either from the start of treatment or in a sequential fashion after 2 to 5 years of tamoxifen.

As a consequence, the traditional paradigm of sequential use of available endocrine agents for the control of advanced breast cancer is undergoing profound changes. More and more patients, when confronted with metastatic disease, will have already received an anti-aromatase agent in the adjuvant setting. Many of those who have not had an anti-aromatase agent as part of their adjuvant therapy, and receive one as first-line therapy for advanced disease, will need further endocrine manipulation when they progress. In either case, the possibility of re-challenging with an anti-aromatase agent is attractive: if further benefit can be obtained from these low-toxicity drugs, patients could be spared other treatments until further progression.

Anti-aromatase drugs can be divided into two categories, steroidal inactivators and nonsteroidal inhibitors, based on their chemical structure and mechanism of action [13]. Exemestane (and the parent compound 4-hydroxyandrostenedione, or formestane) has a 
steroidal structure closely resembling the natural substrate (androstenedione) of the aromatase enzyme, which is irreversibly inactivated as a result of covalent binding. Non-steroidal aromatase inhibitors (which include the first-generation agent aminoglutethimide, second-generation vorozole and fadrozole, and the third-generation agents letrozole and anastrozole) bind to the heme part of aromatase, and the inhibition is reversible. The clinical significance of these differences is unclear, as all modern antiaromatase agents result in profound suppression of oestrogen levels in postmenopausal women, which is believed to be their principal mechanism of action against breast cancer. Despite this, there is clinical evidence of activity of steroidal inactivators after failure of non-steroidal inhibitors [14-18], suggesting a degree of non-cross resistance between the two classes of anti-aromatase agents. The opposite sequence, i.e. nonsteroidal inhibitors after a steroidal inactivator, has been explored in a study of anastrozole in patients who had previously received formestane [19]. No study has examined so far this sequence using exemestane, which is the steroidal inactivator in current clinical use.

We have designed an exploratory clinical trial (GONO-MIG8) where postmenopausal breast cancer patients were given exemestane as their first anti-aromatase therapy, followed by letrozole or anastrozole at the time of disease progression. Patients who had previously received anti-aromatase treatment were also included in the study, and treated with an agent of the opposite class.

\section{Patients and Methods}

Study design. The study was designed as an open-label, non-randomised multicentre clinical trial. The primary endpoint of the study was to evaluate the Clinical Benefit (CB) rate, defined as the proportion of patients obtaining either a complete response (CR) or a partial response (PR) or stable disease (SD) of $\geq 24$ weeks duration, with exemestane and non-steroidal aromatase inhibitors used in a sequential fashion. The secondary endpoints were time to progression (TTP) and overall survival (OS).

Inclusion criteria. Postmenopausal women with cytologically or histologically proven breast cancer were eligible. Patients had to have advanced (metastatic or inoperable) disease, with at least one measurable or assessable lesion, progressing at the time of recruitment into the study. Postmenopausal status was defined as one of the following: $>12$ months since last menstrual period, or age $>50$ and previous hysterectomy, or surgical or radiation bilateral oophorectomy at any age, or LHRH-induced amenhorrea. Patients were required to have positive or unknown ER and/or PgR status: positivity was defined as $\geq 10 \mathrm{fmol} / \mathrm{mg}$ cytosol protein with the dextran-coated charcoal method, or as $\geq 10 \%$ of positive cells with the immunohistochemistry method. Prior adjuvant or palliative chemotherapy was allowed, provided that the patient had recovered from treatment-related toxicity. Prior radiotherapy was allowed, provided that the patient had measurable or assessable disease in non-irradiated areas. Bisphosphonates were allowed in patients who had measurable or assessable lesions outside the bones. No concomitant investigational agents were allowed during the study.

Prior endocrine therapy with tamoxifen as adjuvant therapy or for advanced disease was allowed but not compulsory. Prior therapy for advanced disease with one anti-aromatase agent was allowed and used for treatment stratification in the study (vide infra), but no 
other prior endocrine therapy for advanced disease was allowed. Additional eligibility criteria included a performance status (ECOG scale) of 0 to 3 and predicted life expectancy $\geq 3$ months. Patients were excluded in case of severe psychiatric conditions precluding adequate compliance with treatment and/or follow up.

The study was approved by the Ethical Committee of the National Cancer Institute in Genova, Italy and by participant centres' local ECs, according to national and institutional guidelines. All patients gave written informed consent for their participation.

Treatment. Upon entry into the study, patients were stratified in 3 groups according to previous exposure to anti-aromatase agents for advanced disease. Patients who had not previously received anti-aromatase treatment were started on exemestane $25 \mathrm{mg}$ once daily (group A). Patients who had received a steroidal aromatase inactivator (i.e., exemestane or formestane) prior to study entry, were started on either letrozole $2.5 \mathrm{mg}$ or anastrozole $1 \mathrm{mg}$ once daily, depending on the investigator's preference (group B). Finally, patients who had previously received letrozole or anastrozole were started on exemestane $25 \mathrm{mg}$ once daily (group C). Treatment in all groups was to be continued until progression: in group A, if further endocrine therapy was considered clinically appropriate, patients were offered a cross-over to letrozole or anastrozole (depending on the investigator's preference) and to continue the study in group B. Further therapy (endocrine or chemotherapy) after disease progression in groups B and C was not specified by the protocol, but given according to individual clinicians' judgement.

Assessments. Baseline assessments included a complete clinical history, physical examination, haematology, blood chemistry, chest X-rays, abdominal CT-scan or ultrasound, and bone scan. Tumour assessments were repeated using the same diagnostic modalities every 3 months. Response assessment was based on modified World Health Organization criteria. Complete response (CR) was defined as the disappearance of all known lesions without any new lesions. Partial response (PR) was defined as at least a $50 \%$ decrease in the sum of the product of the perpendicular diameters of all measurable lesions, without any new lesions or progression of existing lesions. Objective responses had to be confirmed by a second assessment performed 4 weeks or longer after the first assessment: external audit of responses was not performed in this study. Progressive disease (PD) was defined by a $25 \%$ or greater increase in the size (products of the diameters) of one or more lesions, or the appearance of any new lesions. SD was defined as a less than $50 \%$ decrease or a less than $25 \%$ increase in measurable disease without any new lesions, the stabilization of assessable disease for 8 or more weeks without any new lesions, or failure to meet the criteria for $\mathrm{CR}, \mathrm{PR}$, or PD. Side effects were assessed using the NCI Common Toxicity Criteria.

Statistical methods. A recruitment of 50-70 patients was considered adequate to allow exploratory analyses, as the study was not comparative. Time to progression and overall survival were analysed by means of the Kaplan-Meier method. TTP was defined as the time from starting an anti-aromatase agent in the study to progression, or death from any cause, or censored at the last day of follow up for patients who had not progressed at the date of analysis (November 2004). In patients who were crossed-over from exemestane to a non-steroidal aromatase inhibitor upon progression, TTP was calculated separately for the two treatments. OS was defined as the time from starting the first treatment in the study to death from any cause, or censored at the last day of follow up for patients who were alive at the date of analysis.

Descriptive statistics were used for presenting other variables. 


\section{Results}

Seventy patients were recruited between June 2000 and November 2003: six were found to be ineligible (one because of prior treatment with both steroidal and non-steroidal anti-aromatase agents, one because of male sex, and four because they were still on their first anti-aromatase agent and had not yet progressed) and were excluded from the study. Table 1 summarizes patient characteristics at study entry. According to the study design, 40 patients who had never previously received an aromatase inhibitor were started on exemestane $25 \mathrm{mg}$ daily (group A). Twenty-four patients had prior exposure to anti-aromatase agents and were treated in the study with another agent of the opposite

\section{TABLE 1}

Patient characteristics at baseline.

\begin{tabular}{ll}
\hline Total no. of patients & 64 \\
\hline Age (years) & 67 \\
Median & $(47-86)$ \\
Range & $31(48.4)$ \\
\hline ECOG Performance Status (\%) & $23(35.9)$ \\
0 & $6(9.4)$ \\
1 & $2(3.1)$ \\
2 & $2(3.1)$ \\
3 & \\
Unknown & 26.0 \\
\hline Disease Free Interval (months) & $(0-215)$ \\
Median & \\
Range & $51(79.7)$ \\
\hline Hormone receptor status (\%) & $13(20.3)$ \\
Positive & $34(53.1)$ \\
Unknown & $16(25.0)$ \\
\hline Node-positive at diagnosis $(\%)$ & \\
Positive & $11(17.2)$ \\
Unknown & $28(43.8)$ \\
\hline Previous tamoxifen (\%) & $28(43.8)$ \\
No & \\
Adjuvant & $24(37.5)$ \\
For advanced disease & $31(48.4)$ \\
\hline Previous chemotherapy $(\%)$ & $26(40.6)$ \\
Adjuvant & $24(37.5)$ \\
For advanced disease & $14(21.9)$ \\
\hline Dominant site of disease $(\%)$ & $40(62.5)$ \\
Bone & $23(35.9)$ \\
Viscera & $1(1.6)$ \\
\hline Soft tissue & \\
Previous anti-aromatase treatment & \\
None & \\
Steroidal & \\
\hline & \\
\hline
\end{tabular}


class: one patient who had received exemestane was started on anastrozole (group B), and 23 patients who had received a non-steroidal aromatase inhibitor were started on exemestane (group C). Patients' characteristics in the subgroups were similar to those shown in Table 1 for the whole study population, with the exception of the proportion of hormone receptor unknown patients which was $5 / 40(12.5 \%)$ in group A and 8/23 $(34.8 \%)$ in group $C$.

Results with exemestane used as first anti-aromatase agent. In group A ( $1=40), 11$ patients $(27.5 \%)$ had a partial response, $18(45 \%)$ had a stabilization of disease which in 16 patients $(40 \%)$ lasted $\geq 24$ weeks, and 11 progressed $(27.5 \%)$. Thus, the clinical benefit rate for exemestane used as first anti-aromatase agent was $67.5 \%$ (95\% C.I., $52.9 \%$ $82.0 \%$ ) (Table 2), with a median time to progression of 9.6 months and a median overall survival of 29.7 months. In the subgroup of patients who received exemestane without prior treatment with tamoxifen for advanced disease (i.e., as first-line endocrine therapy for advanced disease, $n=23)$, eight patients $(34.8 \%)$ achieved a partial response, and 10 $(43.5 \%)$ a stable disease lasting $\geq 24$ weeks, for a CB rate of $78.3 \%$ ( $95 \%$ C.I., $61.4 \%$ $95.1 \%)$.

TABLE 2

Response.

\begin{tabular}{llll}
\hline & Group A & $\begin{array}{l}\text { Group B } \\
\text { Letrozole/Anastrozole } \\
\text { after Exemestane }\end{array}$ & $\begin{array}{l}\text { Group C } \\
\text { Exemestane after } \\
\text { Letrozole/Anastrozole }\end{array}$ \\
\hline $\begin{array}{l}\text { Number } \\
\text { Response (\%): }\end{array}$ & 40 & 18 & 23 \\
$\begin{array}{l}\text { Complete Response } \\
\text { Partial Response }\end{array}$ & 0 & $2(11.1)$ & 0 \\
$\begin{array}{l}\text { Stable Disease (SD) } \\
\text { Progressive Disease }\end{array}$ & $11(27.5)$ & $2(11.1)$ & $2(8.7)$ \\
Not Evaluable & $18(45.0)$ & $9(50.0)$ & $9(39.1)$ \\
& $11(27.5)$ & $5(27.8)$ & $11(47.8)$ \\
SD $\geq 24$ weeks & 0 & 0 & $1(4.3)$ \\
Clinical Benefil & $16(40.0)$ & $6(33.3)$ & $8(34.8)$ \\
& $27(67.5)$ & $10(55.6)$ & $10(43.5)$ \\
\hline
\end{tabular}

$\mathrm{CR}=$ Complete Response; $\mathrm{PR}=$ Partial Response; $\mathrm{SD}=\mathrm{Stable}$ Disease (no change); $\mathrm{PD}=\mathrm{Progressive}$ Disease; Clinical Benefit $=C R+P R+S D \geq 24$ weeks

Results with letrozole or anastrozole used after exemestane. One patient who had received exemestane for advanced disease prior to study entry, and 17 patients who started treatment with exemestane in group A and eventually progressed, received second-line anti-aromatase therapy with a non-steroidal aromatase inhibitor (letrozole, $n=17$, or anastrozole, $n=1)$. Two patients $(11.1 \%)$ had a complete response, two $(11.1 \%)$ a partial response, nine $(50 \%)$ had a stabilization of disease, which in six patients $(33.3 \%)$ lasted $\geq 24$ weeks, and five $(27.8 \%)$ progressed. Thus, the $\mathrm{CB}$ rate for nonsteroidal aromatase inhibitors used after exemestane was 55.6\% (95\% C.I., 32.6\% $78.5 \%$ ), with a median time to progression of 9.3 months. As this group was mainly made up of patients originally from group A, median overall survival was superimposable at 29.7 months. Clinical benefit with non-steroidal inhibitors was observed both in 
patients who had previously obtained a $\mathrm{CB}$ with exemestane $(6 / 12,50 \%)$ and in those who had not $(4 / 6,66.6 \%)$.

Results with exemestane used as second anti-aromatase agent. In group $\mathrm{C}(\mathrm{n}=23), 2$ patients $(8.7 \%)$ had a partial response, $9(39.1 \%)$ had a stabilization of disease, which in 8 patients $(34.8 \%)$ lasted $\geq 24$ weeks, and 11 progressed $(47.8 \%)$. Thus, the $\mathrm{CB}$ rate for exemestane used after non-steroidal inhibitors (letrozole in 12 patients, anastrozole in 11 patients) was $43.5 \%$ (95\% C.I., $23.2 \%-63.7 \%$ ), with a median time to progression of 5.1 months and a median overall survival of 27.2. Clinical benefit with exemestane was observed both in patients who had previously obtained a CB with first-line nonsteroidal inhibitors $(8 / 14,57.1 \%)$ and in those who had not $(2 / 9,22.2 \%)$.

Toxicity. Side effects with exemestane used as first or second anti-aromatase agent were assessed in 63 patients and included grade 1 arthralgia in 5 patients (7.9\%), grade 1 hot flushes in $6(9.5 \%)$, drowsiness in $2(3.2 \%)$, weight gain in $2(3.2 \%)$. Two patients (3.2\%) interrupted treatment with exemestane before progression: one for patient's refusal, and one because of a myocardial infarction. Side effects in 18 patients treated with anastrozole or letrozole after exemestane included grade 1-2 hot flushes in 3 patients $(16.7 \%)$, skin rash in one patient $(5.5 \%)$ and dizziness in one patient $(5.5 \%)$. No patient interrupted treatment for side effects.

\section{Discussion}

The possibility of successfully using a second anti-aromatase agent after failure of prior anti-aromatase treatment was first reported by Murray and Pitt in 1995 [14]. In their study, 112 postmenopausal women with advanced breast cancer, who had previously received the non-steroidal inhibitor aminoglutethimide, were treated with 4hydroxyandrostenedione (formestane), a steroidal aromatase inactivator: $21 \%$ had a partial response and $22 \%$ had a stabilization of disease. Patients responded to formestane irrespective of whether they had responded or not to prior aminoglutethimide. Similar results were later reported by Thurlimann et al [15] in 80 patients who received the oral steroidal aromatase inactivator exemestane, $200 \mathrm{mg}$ daily, after progression on aminoglutethimide. The overall response rate (complete response + partial response) was $26 \%$, and the clinical benefit rate (CR $+P R+S D \geq 24$ weeks) was $39 \%$. Again, prior resistance to aminoglutethimide did not exclude benefit from exemestane.

Because formestane and exemestane are more powerful inhibitors of aromatase than aminoglutethimide, a further reduction of peripheral oestrogen levels was considered as a possible explanation for the partial lack of cross-resistance observed in the above studies. However, a subsequent trial by Lonning et [16] al showed that exemestane can also induce tumor regressions and prolonged stabilization after prior exposure to letrozole, anastrozole or vorozole, which are all more powerful than aminoglutethimide. In their study, 241 women received exemestane $25 \mathrm{mg}$ daily (the dose currently used in clinical practice) after failure of non-steroidal inhibitors, with an overall response rate (as assessed by an independent review panel) of $6.6 \%$ and a clinical benefit rate of $24.3 \%$. Results in the subgroup of patients $(n=105)$ who had previously received letrozole, anastrozole or vorozole were similar $(\mathrm{ORR}=4.8 \%$ and $\mathrm{CBR}=20.4 \%)$ : in these patients, serum oestrogen levels were already suppressed by the previous agent, and the 
cross-over to exemestane did not result in further detectable reduction of oestrogens. In a recent trial by Iaffaioli et al [17], exemestane $25 \mathrm{mg}$ daily was used in 50 postmenopausal women with metastatic breast cancer previously treated with anastrozole: the objective response rate with exemestane was $8 \%$ and the clinical benefit rate $44 \%$, remarkably similar to what we observed in our study $(8.7 \%$ objective response rate and $43.5 \%$ clinical benefit rate) in 23 patients who had previously been treated with letrozole or anastrozole and received exemestane as their second anti-aromatase agent. Carlini et al [18] also reported a 43\% clinical benefit rate with exemestane in 30 metastatic breast cancer patients pretreated with letrozole or anastrozole.

Thus, sufficient clinical evidence suggests that a steroidal inactivator (exemestane in today's practice) can be offered to patients who have already received letrozole or anastrozole, with a good chance to achieve further disease control. Can we assume that the opposite sequence is also valid? Only one study so far has examined a sequence where a steroidal inactivator is used as first anti-aromatase agent. Harper-Wynne and Coombes [19] reported in 1999 on 21 patients who had received prior formestane, and were treated with anastrozole as second anti-aromatase agent. Although anastrozole did not result in objective responses, $62 \%$ of the patients had stabilization of disease, and in $24 \%$ of patients disease stabilization lasted $>6$ months.

Our study is the first to report on the use of exemestane as first anti-aromatase agent, with a planned crossover to anastrozole or letrozole at the time of progression. The clinical benefit rate with exemestane was encouragingly high $(67.5 \%$ overall): in patients who had not had prior tamoxifen for advanced disease (i.e., receiving exemestane as first-line endocrine therapy) clinical benefit reached $78.3 \%$ with objective responses in $34.8 \%$ of patients, which compares well with the objective response rate of $45 \%$ reported by Paridaens et al in a phase III study of first-line exemestane versus tamoxifen [4]. The median time to progression in our study was more than 9 months: at the time of the analysis, $15 \%$ of the patients had been on exemestane for $>1$ year and had not yet progressed. This helps explain why the number of patients who eventually were crossed-over to letrozole or anastrozole is relatively small $(n=18)$. In this group however, the non-steroidal inhibitors obtained two CRs and two PRs, for an overall objective response rate of $22.2 \%$ : six additional patients $(33.3 \%)$ obtained $S D \geq 24$ weeks, translating into a total clinical benefit rate of $55.6 \%$. Although a larger trial would be necessary to accurately define the extent of benefit achievable with nonsteroidal inhibitors after exemestane, our study provides a proof of the concept that the partial lack of cross-resistance between aromatase inhibitors and inactivators is independent of the sequence used.

The pharmacological and molecular bases for this phenomenon remain unclear. Although anastrozole, letrozole and exemestane all cause profound estrogen suppression [20], subtle differences in the degree of suppression could be of importance for clinical outcome. A randomized, cross-over comparison of letrozole and anastrozole, showing more potent suppression of total-body aromatization and plasma estrogen levels for letrozole, has been conducted [21]: no such direct comparison however is available for steroidal versus non steroidal anti-aromatase agents. Intra-tumoral aromatase may be an important additional target for inhibition [22], and differences between the agents at this level cannot be excluded. Moreover, the administration of exemestane can result in a weak androgenic activity through its metabolite 17-hydroxyexemestane [23]. 
Such activity, which is lacking in non-steroidal aromatase inhibitors, may also represent a difference relevant to sequencing. About $70-80 \%$ of all breast cancers express the androgen receptor: androgens have been shown to have antiproliferative effects on breast cancer cell lines [24], and clinical responses with androgen compounds have been reported in the past [25].

Regardless of the potential mechanisms involved, the possibility of using anti-aromatase agents twice during the course of a patient's natural history has important clinical implications that deserve to be investigated further. Although this was not directly addressed by our study, or by the other trials looking at sequential anti-aromatase treatment, the partial lack of cross-resistance suggests for example that patients with advanced breast cancer could still benefit from an anti-aromatase agent of the opposite class, even if they received anti-aromatase treatment as part of their adjuvant therapy. Another area of potential interest would be to find the right place in the sequence for other endocrine treatments, such as fulvestrant [26], or tamoxifen if not used previously. Since one of the goals of endocrine therapy in patients with hormone-sensitive advanced breast cancer is to prolong the control of the disease for as long as possible, further refinements in the sequential use of available agents may result in increased patient benefit and delay the need for chemotherapy.

\section{References}

1. Nabholtz JM, Buzdar A, Pollak M et al. Anastrozole is superior to tamoxifen as first-line therapy for advanced breast carcinoma in postmenopausal women: results of a North American multicenter randomized trial. J Clin Oncol 2000; 18: 3758-67.

2. Bonneterre J, Thurlimann B, Robertson JF, et al. Anastrozole versus tamoxifen as first-line therapy for advanced breast cancer in 668 postmenopausal women: results of the Tamoxifen or Arimidex Randomized Group Efficacy and Tolerability study. J Clin Oncol 2000;18: 3748-57.

3. Mouridsen H, Gershanovich M, Sun Y et al. Superior efficacy of letrozole versus tamoxifen as first-line therapy for postmenopausal women with advanced breast cancer: results of a phase III study of the International Letrozole Breast Cancer Group. J Clin Oncol 2001; 19: 2596-606.

4. Paridaens R, Therasse P, Dirix L, et al. First line hormonal treatment (HT) for metastatic breast cancer $(\mathrm{MBC})$ with exemestane $(\mathrm{E})$ or tamoxifen $(\mathrm{T})$ in postmenopausal patients (pts) - A randomized phase III trial of the EORTC Breast Group. J Clin Oncol 2004:22:14s (abs 515)

5. Eiermann W, Paepke S, Appfelstaedt $J$ et al. Preoperative treatment of postmenopausal breast cancer patients with letrozole: a randomized double-blind multicenter study. Ann Oncol 2001; 12: 1527-32.

6. Semiglazov VF, Semiglazov VV, Ivanov VG et al. Neoadjuvant endocrine therapy: exemestane (E) vs tamoxifen (T) in postmenopausal ER+ breast cancer patients (T1-4N1-2MO). Breast Cancer Res Treat 2003; 83(suppl 1): S22 (Abstract 111).

7. Dixon JM, Renshaw L, Bellamy C, et al. The effects of neoadjuvant anastrozole (Arimidex) on tumor volume in postmenopausal women with breast cancer: a randomized, double-blind, single-center study. Clin Cancer Res 2000;6:2229-35. 
8. The ATAC (Arimidex, Tamoxifen Alone or in Combination) Trialists Group. Anastrozole alone or in combination with tamoxifen versus tamoxifen alone for adjuvant treatment of postmenopausal women with early breast cancer: first results of the ATAC randomized trial. Lancet 2002; 359:2131-9.

9. Goss PE, Ingle JN, Martino $S$ et al. A randomized trial of letrozole in postmenopausal women after five years of tamoxifen therapy for early-stage breast cancer. $\mathrm{N}$ Engl J Med 2003; 349: 1793-802.

10. Coombes RC, Hall E, Gibson LJ et al. A randomized trial of exemestane after two to three years of tamoxifen therapy in postmenopausal women with primary breast cancer. $\mathrm{N}$ Engl $\mathrm{J}$ Med 2004; 350: 1081-92.

11. Thürlimann B et al. BIG 1-98: A Prospective Randomized Double-Blind Double-Dummy Phase III Study to Evaluate Letrozole as Adjuvant Endocrine Therapy for Postmenopausal Women with Receptor-Positive Breast Cancer. 9th St Gallen Breast Cancer Conference, 2005

12. Winer EP, Hudis $\mathrm{C}$, Burstein $\mathrm{HJ}$, et al. American society of clinical oncology technology assessment on the use of aromatase inhibitors as adjuvant therapy for postmenopausal women with hormone receptor-positive breast cancer: status report 2004. J Clin Oncol 2005; 23:619-29.

13. Lonning PE. Aromatase inhibitors in breast cancer. Endocr Relat Cancer 2004;11:179-89.

14. Murray R, Pitt P. Aromatase inhibition with 4-OHAndrostenedione after prior aromatase inhibition with aminoglutethimide in women with advanced breast cancer. Breast Cancer Res Treat 1995;35:249-253

15. Thurlimann B, Paridaens R, Serin D, et al. Third-line hormonal treatment with exemestane in postmenopausal patients with advanced breast cancer progressing on aminogluthetimide: a phase II multicentre multinational study. Eur J Cancer 1997;33:1767-73

16. Lønning PE, Bajetta E, Murray R et al. Activity of exemestane (Aromasin(B) in metastatic breast cancer after failure of nonsteroidal aromatase inhibitors: a phase II trial. J Clin Oncol $2000 ; 18: 2234-44$.

17. Iaffaioli RV, Formato R, Tortoriello A, et al. Phase II study of sequential hormonal therapy with anastrozole/exemestane in advanced and metastatic breast cancer. $\mathrm{Br} \mathrm{J}$ Cancer $2005 ; 92: 1621-1625$

18. Carlini P, Michelotti A, Giannarelli D, et al. Exemestane (EXE) is an effective 3rd line hormonal therapy for postmenopausal metastatic breast cancer (MBC) patients (pts) pretreated with 3rd generation non steroidal aromatase inhibitors (nSAI). Ann Oncol 2002;13 (suppl 5):s48 (abs 171P)

19. Harper-Wynne C, Coombes RC. Anastrozole shows evidence of activity in postmenopausal patients who responded or stabilised on formestane therapy. Eur J Cancer 1999; 35: 744-6.

20. Lønning PE. Aromatase inhibitors in breast cancer. Endocr Rel Cancer 2004;11:179-189

21. Geisler J, Haynes B, Anker G, Dowsett M, Lønning PE. Influence of letrozole and anastrozole on total body aromatization and plasma estrogen levels in postmenopausal breast cancer patients evaluated in a randomized, cross-over study. J Clin Oncol 2002;20:751-7

22. Brodie A, Lu Q, Liu Y, Long B. Aromatase inhibitors and their antitumor effects in model systems. Endocrine-Related Cancer 1999; 6: 205-10

23. Johannessen DC, Engan T, Di Salle E, Zurlo MG, Paolini J, Ornati G, Piscitelli G, Kvinnsland S, Lonning PE. Endocrine and clinical effects of exemestane (PNU 155971), a novel steroidal aromatase inhibitor, in postmenopausal breast cancer patients: a phase I study. Clin Cancer Res 1997;3:1101-8. 
24. Ando S, De Amicis F, Rago V et al. Breast cancer: from estrogen to androgen receptor. Mol Cell Endocrinol 2002; 193: 121-8

25. Pronzato P, Amoroso D, Ardizzoni A, Bertelli G, Conte PF, Michelotti A, Rosso R. A phase II study with danazol in metastatic breast cancer. Am J Clin Oncol 1987; 10:407-9

26. Bundred N. Preclinical and clinical experience with fulvestrant (Faslodex) in postmenopausal women with hormone receptor-positive advanced breast cancer. Cancer Invest $2005 ; 23: 173-81$. 


\section{Chapter 10}

Conclusions:

New paradigms in hormonal therapy of breast cancer

Modified version of:

Gicanfiliph" Bertelli

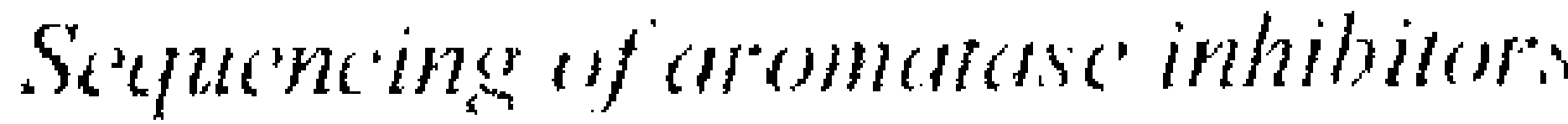

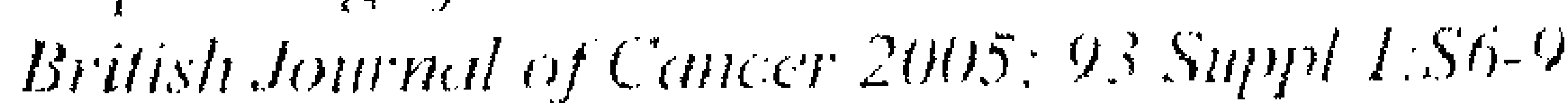


The research papers presented in this Thesis are focused on the two main types of endocrine manipulation used in hormone-sensitive breast cancer: competition at the level of the estrogen receptor by antiestrogens, and inhibition of the conversion of steroidal precursors into estrogens by inhibitors of the aromatase enzyme. The latter strategy (only applicable in postmenopausal women) has gained momentum with the development of third-generation agents, more potent and tolerable than the original compound aminogluthethimide.

One of the paradigms of hormonal therapy of breast cancer is that patients may benefit from more than one line of treatment during their natural history. The sequence of treatments was until recently well established: tamoxifen was the only agent approved for adjuvant therapy of hormone-sensitive early breast cancer, and at the time of relapse, postmenopausal patients with tamoxifen-resistant disease (progressing during adjuvant tamoxifen or within one year from its discontinuation) were generally offered an aromatase inhibitor (AI), followed by a progestin (usually megestrol acetate) when they further progressed.

Tamoxifen however is not devoid of side effects, as illustrated in Chapters 1-5 of the Thesis, and its efficacy is limited by de novo or acquired resistance resulting in disease relapse or progression. The evidence of better tolerability and efficacy of AIs in advanced disease has stimulated research on their use in the adjuvant setting. This has led to developments, some of which reported in Part 2 of the Thesis, that impose today a reconsideration of the paradigms of sequential use of hormonal agents mentioned above. Following the early results of the Arimidex, Tamoxifen Alone or in Combination (ATAC) study [1], anastrozole has become an alternative to tamoxifen in the early adjuvant setting (years 0-5). Moreover, early results of the MA17 trial [2] and of the Intergroup Exemestane Study [3] (Chapters 6 and 7) suggest that a sequence of tamoxifen followed by an AI may be beneficial in the early or in the extended adjuvant setting (years 5-10), respectively.

Further results of ongoing adjuvant trials are eagerly awaited, but it is likely that in the future an increasing number of patients who present with advanced breast cancer will already have received an $\mathrm{AI}$ (with or without tamoxifen) as part of their adjuvant therapy. The remaining patients will have had tamoxifen only; and a few will be hormonetherapy naîve. After taking into account previous treatments, the choice of an appropriate hormonal therapy should be based on the evidences regarding tolerability and efficacy of the available agents.

Before trying to determine where should each agent fit in the sequence of therapy, however, it may be useful to consider that the alternative strategy, i.e. combination therapy, does not probably have advantages over the use of single agents. The recently completed ATAC study found that the combination of anastrozole and tamoxifen was no more effective than either agent alone. There was a statistically significant improvement in disease-free survival at 3 years for anastrozole compared with tamoxifen (hazard ratio [HR] 0.83), but not for combination therapy compared with tamoxifen (HR 1.02). In women with oestrogen-receptor positive tumours, the HR was 0.78 in favour of anastrozole compared with tamoxifen or combination therapy. The recently published Intergroup Exemestane Study did not explore combined treatments, but its results support the sequential use of tamoxifen and AIs. Switching to exemestane after 2-3 years of tamoxifen therapy resulted in a significant reduction in disease-free survival events (HR 0.68; $p<0.001$ ) compared with continuing on tamoxifen [3]. Similarly, sequential 
use of letrozole given after 5 years of tamoxifen showed improved disease-free survival vs placebo in the MA17 trial [2].

Given that the use of single agents appears preferable, should tamoxifen or AIs be used as first-line treatment in a hormone-therapy naive patient, or in a patient who has completed adjuvant tamoxifen more than 12 months before relapse (i.e., in a potentially tamoxifen-sensitive patient)? The improved efficacy (time to progression) and sideeffect profile of AIs compared with tamoxifen [4-7] suggest that these agents should be used first. This is reinforced by the results of studies comparing AIs and tamoxifen in the neoadjuvant setting [8-11]. AIs are also considered the agents of first choice for patients with tamoxifen-resistant disease, i.e. those who progress during or within one year from adjuvant tamoxifen.

A more problematic choice is faced for a patient who has already received an $\mathrm{AI}$ as part of her adjuvant therapy. Is there a role for further treatment with an $\mathrm{AI}$ in such a patient? The same question arises at the time of second progression after treatment with AIs in the advanced disease setting.

\section{RATIONALI: FOR SEQUENCING OF AIS}

In both cases, the goal of continuing endocrine therapy for as long as possible is to prolong the control of the disease and delay the need for chemotherapy, thus also delaying its associated toxicity. Based on structural differences and different mechanisms of action among the AIs, a lack of cross-resistance between steroidal and non-steroidal AIs has been hypothesized, thus justifying the use of different AIs in the same patient during the course of her disease.

The steroidal inactivator, exemestane, has a structure very similar to the androgen substrate for aromatase, androstenedione; its covalent binding to aromatase results in irreversible inactivation of the enzyme. By contrast, non-steroidal AIs bind to the heme part of aromatase, and the inhibition is reversible. It is uncertain, however, whether this structural difference results in significant clinical differences. Both steroidal and nonsteroidal AIs effectively reduce serum oestrogen concentrations in postmenopausal patients. Intra-tumoral aromatase may however be an important additional target for inhibition [12], and differences between the AIs at this level cannot be excluded. Moreover, the partial androgen activity of exemestane, which is lacking in the non-steroidal AIs, may also represent a difference relevant to sequencing. About $70-80 \%$ of all breast cancers express the androgen receptor, and androgens have been shown to have antiproliferative effects on breast cancer cell lines [13].

Although the pharmacological and molecular bases for their lack of cross-resistance need to be better investigated, the possibility of successfully using steroidal and nonsteroidal AIs in sequence is supported by clinical studies [14-17]. The largest of these studies, which examined exemestane treatment in patients who had failed on nonsteroidal AIs found that $6.6 \%$ of patients achieved an objective response and 17.4 prolonged disease stabilization ( $\geq 24$ weeks), which translates to a $24 \%$ total clinical benefit rate [14]. 


\section{SELECTION OF THE OPTIMAL. SEQUENCE OF AIS}

If sequential application of Als enables prolonged hormonal treatment, in which order should these agents be given? The main choice would appear to be whether to give a steroidal AI before a non-steroidal AI, or vice versa. However, as yet, clear clinical differences between these two types have not emerged, nor is it known whether their efficacy varies in different patient groups. There may, however, be differences in sideeffect profiles between the steroidal and non-steroidal AIs, which may become clearer with further clinical trial results. The main adverse events of hormonal treatments in breast cancer are hot flushes, gastrointestinal (e.g., nausea and vomiting) and gynaecological events (e.g., vaginal bleeding/discharge). In addition, effects have been reported on bone density, lipid levels and thromboembolic risk. . In the ATAC trial, there was a significantly lower incidence of hot flushes, vaginal bleeding and discharge and venous thromboembolism with anastrozole than tamoxifen but anastrozole was associated with more musculoskeletal symptoms and fractures [1]. In addition, letrozole has been associated with an increase in bone-resorption markers in plasma and urine [18]. By contrast, early results from animal models and postmenopausal volunteers suggest the steroidal AI, exemestane, may have a bone-sparing effect compared with non-steroidal agents $[19,20]$. There is also evidence that exemestane does not adversely affect blood lipids [20,21], whereas this may not be the case with the non-steroidal AIs [22].

The current practice is to use anastrozole or letrozole before exemestane. This is based on the magnitude of the available database and clinical experience with anastrozole and letrozole compared with exemestane. Some evidences, however, suggest that the opposite sequence is equally valid.

\section{STEROIDAL AI FOLLOWED BY NON-STEROIDAL AIS}

The GONO (Gruppo Oncologico Nord Ovest) MIG-8 trial was designed to assess treatment with different $\mathrm{AI}$ sequences in postmenopausal patients with estrogen receptor-positive or -unknown advanced breast cancer. This is a prospective, nonrandomized study where patients not previously treated with AIs received exemestane first, and were crossed over to a non-steroidal AI (anastrozole or letrozole) at the time of progression. Patients with previous exposure to AIs received the alternative AI after entering the study (exemestane for patients pre-treated with a non-steroidal AI; letrozole or anastrozole for patients pre-treated with a steroidal AI. The results were published [15] and are presented in Chapter 9. The clinical benefit rate (objective responses + stable disease $\geq 24$ weeks) among 18 patients receiving a non steroidal AI after exemestane was $55.6 \%$ with a median TTP of 9.3 months.

Harper-Wynne and Coombes reported on a group $(n=21)$ of patients who had received the steroidal AI formestane and were treated with anastrozole [17]. Overall, $62 \%$ of the patients stabilized on anastrozole: when only those who had responded to formestane $(n=12)$ are considered, $78 \%$ achieved further stable disease on anastrozole [17].

\section{NON-STEROIDAL AIS FOLLOWED BY STEROIDAL AI}

The other AI sequence assessed in the GONO-MIG-8 trial was exemestane following treatment with a non-steroidal AI (letrozole or anastrozole). In the final analysis, clinical benefit was obtained by $43.5 \%$ of 23 patients receiving exemestane as second antiaro- 
matase agent, with a median TTP of 5.1 months. A clinical benefit rate of $20.4 \%$ was reported by Lonning et al. with exemestane in 105 patients after failure of thirdgeneration non-steroidal AIs [14]. The median duration of clinical benefit was 37 weeks. Interestingly, exemestane was also associated with responses in patients who had not responded to prior hormone therapy [14].

Carlini et al. conducted a retrospective study on patients receiving formestane after anastrozole or letrozole $(n=20)$ [16]. The clinical benefit rate was $55 \%$, with a median duration of 15 months.

\section{ONGOING TRIALS}

There are several large trials currently in progress or planned which should throw more light on whether there is an optimal sequence for AI therapy. These include trials of formestane followed by exemestane or anastrozole (SAINT), of exemestane followed by anastrozole or vice versa (GEICAM 2002-05) and non-steroidal AIs followed by fulvestrant or exemestane in patients failing non-steroidal AIs (SOFEA)

Trials in progress include the NCIC MA27 and the SOFEA trials. NCIC MA27 is a randomized phase III trial comparing exemestane (with or without celecoxib) with anastrozole (with or without celecoxib) for 5 years in preventing cancer recurrence in postmenopausal women following surgery for primary breast cancer. The study aims to recruit 6800 women. The SOFEA trial is comparing fulvestrant with or without anastrozole versus exemestane in 750 patients failing non-steroidal AIs.

\section{OTHER CONSIDERATIONS}

The impact of new agents, such as fulvestrant, on drug sequencing will also need to be considered. Preliminary results from studies of fulvestrant after progression on tamoxifen and anastrozole reported a clinical benefit rate of $34-50 \%$. A study has examined fulvestrant in women who had progressed on tamoxifen and an AI [23]. Of 20 patients who had progressed after tamoxifen and a non-steroidal AI, two showed a partial response and five had stable disease for $\geq 24$ weeks, which represents a clinical benefit rate of $41 \%$. An open, parallel-group trial compared fulvestrant and anastrozole in postmenopausal women with advanced breast cancer progressing after prior endocrime treatment [24]. Clinical benefit rates were $44.6 \%$ for fulvestrant and $45.0 \%$ for anastrozole, median time to progression was 5.5 months for fulvestrant and 5.1 months for anastrozole. Similar results were reported in another trial of the same design [25].

\section{Conclusions}

Current evidence suggests that there is clinical utility in using anti-aromatase agents in sequence (steroidal inactivators after non-steroidal inhibitors, or vice-versa). In other words, anti-aromatase drugs of the two types can be used at different times to prolong disease control, before changing to other, less tolerable treatments (such as progestins or chemotherapy). The traditional sequence of hormone therapy, which is to say, adjuvant tamoxifen followed by an AI as first line therapy at relapse and then progestins as second line could therefore change to adjuvant tamoxifen followed by an AI as first line therapy at relapse and then another AI as second line and finally, progestins as third line 
therapy. The above sequence is likely to change further based on the results of recent and ongoing adjuvant trials (e.g. ATAC, MA-17, BIG FEMTA, ARNO, IES, TEAM), which may result in an increased number of patients exposed to AIs in the adjuvant setting. The choice of first- and subsequent-line hormone therapy for advanced disease will be based on the treatment history of the patient, on the (limited) data reported above on non-cross resistance, and on a balance of the expected benefits and toxicities with the agent of choice.

\section{References}

1. The ATAC (Arimidex, Tamoxifen Alone or in Combination) Trialists Group. Anastrozole alone or in combination with tamoxifen versus tamoxifen alone for adjuvant treatment of postmenopausal women with early breast cancer: first results of the ATAC randomized trial. Lancet 2002; 359: 2131-9.

2. Goss PE, Ingle JN, Martino S et al. A randomized trial of letrozole in postmenopausal women after five years of tamoxifen therapy for early-stage breast cancer. $\mathrm{N}$ Engl $\mathrm{J}$ Med 2003; 349: 1793-802.

3. Coombes RC, Hall E, Gibson LJ et al. A randomized trial of exemestane after two to three years of tamoxifen therapy in postmenopausal women with primary breast cancer. $\mathrm{N}$ Engl $\mathrm{J}$ Med2004; 350: 1081-92.

4. Mouridsen H, Gershanovich M, Sun $Y$ et al. Superior efficacy of letrozole versus tamoxifen as first-line therapy for postmenopausal women with advanced breast cancer: results of a phase III study of the International Letrozole Breast Cancer Group. J Clin Oncol 2001; 19: 2596-606.

5. Nabholtz JM, Buzdar A, Pollak $M$ et al. Anastrozole is superior to tamoxifen as first-line therapy for advanced breast carcinoma in postmenopausal women: results of a North American multicenter randomized trial. J Clin Oncol 2000; 18: 3758-67.

6. Milla-Santos A, Milla L, Rallo L, Solana V. Anastrozole vs. tamoxifen in hormone dependent advanced breast cancer: a phase II randomized trial. Breast Cancer Res Treat. 2000; 64: Abstract 173.

7. Paridaens R, Therasse P, Dirix L, et al. First results of a randomized phase III trial comparing exemestane versus tamoxifen as first-line hormone therapy (HT) for postmenopausal women with metastatic breast cancer (MBC) - EORCT 10951 in collaboration with the exemestane working group and NCIC clinical trials group. Eur J Cancer 2004; 2:126 (abstract 241)

8. Eiermann W, Paepke S, Appfelstaedt $\mathrm{J}$ et al. Preoperative treatment of postmenopausal breast cancer patients with letrozole: a randomized double-blind multicenter study. Ann Oncol 2001; 12: 1527-32.

9. Ellis MJ, Coop A, Singh B et al. Letrozole is more effective neoadjuvant endocrine therapy than tamoxifen for ErbB-1 and/or ErbB2-positive, estrogen receptor positive primary breast cancer: evidence from a phase III randomized trial. J Clin Oncol. 2001; 19:3808.

10. Semiglazov VF, Semiglazov VV, Ivanov VG et al. Neoadjuvant endocrine therapy: exemestane (E) vs tamoxifen ( $\mathrm{T}$ ) in postmenopausal ER+ breast cancer patients (T1-4N 1-2MO). Breast Cancer Res Treat 2003; 83(supp! 1): S22 (Abstract 111). 
11. Dixon JM, Renshaw L, Bellamy $C$, et al. The effects of neoadjuvant anastrozole (Arimidex) on tumor volume in postmenopausal women with breast cancer: a randomized, double-blind, single-center study. Clin Cancer Res 2000;6:2229-35.

12. Brodic A, Lu Q, Liu Y, Long B. Aromatase inhibitors and their antitumor effects in model systems. Endocrine-Related Cancer 1999; 6: 205-10.

13. Ando $\mathrm{S}$, De Amicis $\mathrm{F}$, Rago $\mathrm{V}$ et al. Breast cancer: from estrogen to androgen receptor. Mol Cell Endocrinol 2002; 193: 121-8.

14. Lonning PE, Bajetta E, Murray $R$ et al. Activity of exemestane (Aromasin $(\hat{\mathbb{B}})$ ) in metastatic breast cancer after failure of nonsteroidal aromatase inhibitors: a phase II trial. J Clin Oncol 2000; 18: 2234-44.

15. Bertelli G, Garrone O, Merlano $M$ et al. Sequential Treatment with Exemestane and NonSteroidal Aromatase Inhibitors in Advanced Breast Cancer. Oncology 2005;69:471-477

16. Carlini P, Frassoldati A, De Marco $S$ et al. Formestane, a steroidal aromatase inhibitor after failure of non-steroidal aromatase inhibitors (anastrozole and letrozole): is a clinical benefit still achievable? Ann Oncol 2001; 12:1539-43.

17. Harper-Wynne C, Coombes RC. Anastrozole shows evidence of activity in postmenopausal patients who responded or stabilised on formestane therapy. Eur J Cancer 1999; 35: 744-6.

18. Heshmati HM, Khosla S, Robins SP, O'Fallon WM, Melton LJ III, Riggs BL. Role of low levels of endogenous estrogen in regulation of bone resorption in late postmenopausal women. J Bone Miner Res 2002; 17: 172-8.

19. Goss PE, Thomsen $\mathrm{T}$, Banke-Bochita $\mathrm{J}$ et al. Elfects of steroidal and nonsteroidal aromatase inhibitors on markers of bone turnover and lipid metabolism in healthy volunteers. 26th Annual San Antonio Breast Cancer Symposium, December 2-6, 2003, San Antonio, Texas, Abstract No. 427.

20. Goss P, Grynpas M, Qi S, Hu H. The effects of exemestane on bone and lipids in the ovariectomized rat [abstract]. Breast Cancer Res Treat 2001; 69:224.

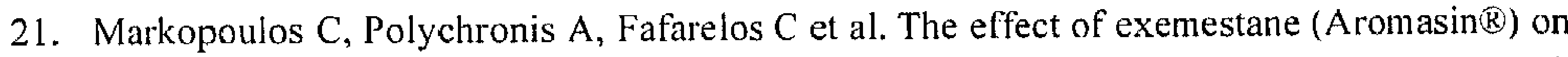
the lipidemic profile of breast cancer patients: preliminary results of the TEAM trial Greek sub-study. 26th Annual San Antonio Breast Cancer Symposium December 3-6, 2003 (abstract 440)

22. Elisaf MS, Bairaktari ET, Nicolaides C et al. Effect of letrozole on the lipid profile in postmenopausal women with breast cancer. Eur J Cancer 2001; 37: 1510-13.

23. Perey L, Thürlimann B, Hawle $H$ et al. Fulvestrant ('Faslodex') as hormonal treatment in postmenopausal patients with advanced breast cancer progressing after treatment with tamoxifen and aromatase inhibitors. Breast Cancer Res Treat 2002; 76: 572.

24. Howell A, Robertson JFR, Quaresma Albano J et al. Fulvestrant, formerly ICl 182,780, is as effective as anastrozole in postmenopausal women with advanced breast cancer progressing after prior endocrine treatment. J Clin Oncol 2002; 20: 3395-403.

25. Osborne CK, Pippen J, Jones SE et al. Double-blind, randomized trial comparing the efficacy and tolerability of fulvestrant versus anastrozole in postmenopausal women with advanced breast cancer progressing on prior endocrine therapy: results of a North American trial. J Clin Oncol 2002; 20: 3386-95. 
Summary 
In this Thesis, the results of studies on hormonal therapy of breast cancer, performed at the Medical Oncology Department of the National Cancer Institute in Genova and at the Medical Oncology Unit of the S.Croce Hospital in Cuneo, Italy, were presented. Most of the studies were performed within the framework of the cooperative group GONO (Gruppo Oncologico Nord Ovest), and some have been completed at the South West Wales Cancer Institute in Swansea, UK.

Chapter 1 provides an introduction to the studies and an overview of the questions and problems that have been addressed in the Thesis. These include monitoring and treatment of tamoxifen's estrogenic and antiestrogenic side effects, and the role of aromatase inhibitors in early and advanced breast cancer, as alternatives to tamoxifen or used in a sequential strategy.

Chapter 2 presents the results of a study aimed at assessing the role of pelvic ultrasound to screen for endometrial abnormalities associated with the use of adjuvant tamoxifen. One hundred and sixty four asymptomatic patients (110 on treatment with tamoxifen, $20 \mathrm{mg} /$ day, and 54 controls) were included in the study: in the postmenopausal group, tamoxifen-treated patients had significantly thicker endometria and significantly larger uterine volume than controls. Fifty-four percent of patients on tamoxifen had an endometrial thickness $\geq 5 \mathrm{~mm}$, often with an irregular pattern suggesting the presence of cysts. Ultrasound findings, however, did not correlate with endometrial abnormalities at biopsy. In view of its low specificity and poor positive predictive value, which is probably due to sub-epithelial changes and benign polyps induced by tamoxifen, we concluded that routine ultrasound screening in asymptomatic subjects is not recommended.

Attempts to improve on the specificity of ultrasound screening in patients on tamoxifen have included research on refinements such as the instillation of saline solution into the uterine cavity at the time of transvaginal ultrasound scanning, a technique known as sonohysterography. In Chapter 3, sonohysterography was used in asymptomatic postmenopausal patients on tamoxifen, who exhibited an endometrial thickness of $\geq 8 \mathrm{~mm}$ at transvaginal ultrasound. Forty-one patients entered the study: sonohysterography allowed to identify a normal endometrial cavity in 9 patients $(21.9 \%)$, who could thus be spared a biopsy. In the remaining patients, benign polyps $(n=15,36.6 \%)$ and endometrial atrophy $(n=14,34.1 \%)$ were the most common findings; 3 patients $(7.3 \%)$ had simple hyperplasia. Despite a modest improvement over standard transvaginal ultrasound, the proportion of false positive results remained high and we concluded that there is insufficient evidence to recommend this technique routinely in asymptomatic patients on tamoxifen.

Before the advent of aromatase inhibitors, alternative SERMs (selective estrogen receptor modulators) such as toremifene have been explored as possible alternatives to tamoxifen. In Chapter 4, the results of a crossover study from tamoxifen to toremifene are presented. Twenty postmenopausal breast cancer patients receiving adjuvant tamoxifen, $20 \mathrm{mg} / \mathrm{day}$, were switched to toremifene $60 \mathrm{mg} /$ day and the effects on the uterus were assessed prospectively by transvaginal ultrasound. Toremifene did not however modify previous uterine changes induced by tamoxifen. Subsequent studies by others have confirmed that both drugs cause similar ultrasound changes and vaginal 
symptoms, and phase III trials in the adjuvant setting found that the incidence of endometrial cancer was not different between the two drugs.

Chapter 5 investigates treatments for one of tamoxifen's side effects associated with its antiestrogen action, i.e. menopausal symptoms. The study compared six weeks of lowdose oral megestrol acetate (MA) with three injections (on day 1, 14 and 28) of intramuscular medroxyprogesterone acetate (MPA) for the control of hot flushes in 71 postmenopausal patients with early breast cancer, of whom $73 \%$ were on adjuvant tamoxifen. Six weeks after randomization, a significant reduction (more than $50 \%$ compared to baseline) of the severity and frequency of hot flushes was observed in $67 \%$ and $75 \%$ of the patients randomized to MA and MPA, respectively. Patients treated with medroxyprogesterone acetate had a more prolonged duration of the benefit compared to megestrol, with $89 \%$ of responders still maintaining the effect at week 24 , vs. $45 \%$ in the megestrol group. Today, non-hormonal treatments for hot flushes are usually preferred in breast cancer survivors, based on efficacy data from recent randomized trials: these include the antidepressants venlafaxine, fluoxetine and paroxetine, and the antiepilectic gabapentin. Our study however still provides useful clinical information for patients who do not respond to, or do not tolerate, these agents.

A series of large multicenter trials is currently exploring the role of third-generation aromatase inhibitors in the adjuvant setting, either as an alternative to tamoxifen or in a sequential fashion after tamoxifen. Chapter 6 is a report of the early results of one of such studies, the Intergroup Exemestane Study (IES), an international, phase III, intergroup, randomized, double-blind study which recruited 4742 postmenopausal women with breast cancer who remained disease-free after 2-3 years of adjuvant tamoxifen. Participating patients were randomized to receive either exemestane $25 \mathrm{mg} /$ day or tamoxifen for the remainder of the 5-year period. After a median follow up of 30.6 months, 449 first events (local or metastatic recurrence, contralateral breast cancer, or death) were reported, with an hazard ratio in favor of the exemestane group of 0.68 corresponding to an absolute benefit in terms of disease-free survival of 4.7 percent. The results of this study suggest that five years of postoperative tamoxifen may be suboptimal for postmenopausal patients with estrogen-receptor positive breast cancer, adding strenght to the recommendation that Als should be used in these patients instead or after tamoxifen.

In view of their lack of estrogenic activity, one of the potential advantages of AIs over tamoxifen is a reduction of endometrial toxicity. This is being formally assessed in a sub study within the IES trial, the preliminary results of which are presented in Chapter 7. Two hundred and nineteen patients randomized in the main trial participated to the sub study, and were prospectively monitored with transvaginal ultrasound. Switching to exemestane resulted in a significantly lower proportion of patients with abnormal endometrial thickness two years after randomization $(41.7 \%$ with exemestane vs. $64.6 \%$ with tamoxifen. The mean endometrial thickness and the mean uterine volume were also significantly reduced. The positive changes associated with exemestane were already apparent after 6 months from randomisation.

The last chapters present applications of Als in the setting of metastatic breast cancer. Chapter 8 is a study of maintenance therapy with letrozole in postmenopausal patients 
who responded or stabilized after first-line chemotherapy for advanced breast cancer. Fifty-eight patients were recruited and received letrozole, $2.5 \mathrm{mg}$ /day starting within 8 weeks since the last cycle of chemotherapy. The median time to progression from starting letrozole was 18.5 months. Response status improved during letrozole in $15.5 \%$ of patients who had obtained less than a complete response to chemotherapy. A shorter time to progression was found in patients with abnormal CA 15-3 levels at the start of maintenance letrozole, or with levels increasing $\geq 25 \%$ from baseline during the first six months of letrozole therapy. Maintenance treatment was well tolerated and had no significant impact on quality of life scores. This observation is encouraging, and would warrant further confirmation in a controlled trial.

Chapter 9 is a study of sequential use of exemestane and non-steroidal AIs in advanced breast cancer. Forty patients received exemestane $25 \mathrm{mg}$ daily as first anti-aromatase agent, with a clinical benefit $(\mathrm{CB})$ rate (complete response + partial response + stabilization of disease for $\geq 24$ weeks) of $67.5 \%$ and a median time to progression (TTP) of 9.6 months. In 18 patients, letrozole or anastrozole were used after failure of exemestane: the CB rate was $55.6 \%$ with a median TTP of 9.3 months. In 23 patients, exemestane was used after failure of letrozole or anastrozole: the $\mathrm{CB}$ rate was $43.5 \%$ with a median TTP of 5.1 months.

The study was based on previously reported observations of partial non-cross resistance between steroidal and non-steroidal Ais, but is the first to also examine the sequence of exemestane first, then letrozole or anastrozole. Clinical benefit was observed with both sequences, suggesting that the partial lack of cross-resistance between steroidal and non-steroidal AIs is independent of the sequence used.

As reviewed in Chapter 10, the clinical implications of the sequencing studies are that anti-aromatase drugs of the two types could be used at different times to prolong disease control, before changing to other, less tolerable treatments (such as progestins or chemotherapy). The traditional sequence of hormone therapy is also likely to change further based on the results of recent and ongoing adjuvant trials which may result in an increased number of patients exposed to AIs in the adjuvant setting, and on the introduction of new agents such as the estrogen receptor down-regulator fulvestrant. 
Curriculum Vitae 


\section{DR GIANFILIPPO BERTELLI MD FRCP (EDIN)}

\section{Curricui.um Vitae}

PERSONAL DETALS

Address (work): Oncology Department/South West Wales Cancer Institute, Singleton Hospital, Sketty, Swansea SA2 8QA, UK

Address (home): 5 Uplands Terrace, Swansea SA2 0GU, UK

Telephone \&fax: +44 (0)1792 285826

e-mail: $\quad$ gianfilippo.bertelli@swansea-tr.wales.nhs.uk

Personal page: http://www.medicine.swan.ac.uk/bio-g.bertelli.html

GMC number: 6057041

QUALIFICATIONS

April 1986 MD, University of Genova, Italy

October 1989 Specialist Qualification in Oncology, University of Genova, Italy

June 1994 Specialist Qualification in Internal Medicine, University of Genova, Italy

August 2002 Registration in the General Medical Council (UK) Specialist Register for General (Internal) Medicine

March 2003 Registration in the General Medical Council (UK) Specialist Register for Medical Oncology

October 2003 Fellow of the Royal College of Physicians of Edinburgh

November 2005 ICH- GCP Training

CURRENT POSITION

01/03/03 Full-time Consultant in Medical Oncology, Swansea NHS Trust

01/07/03 Honorary Senior Lecturer in Medical Oncology, School of Medicine, University of Wales Swansea

PREVIOUS APPOINTMENTS

1986-1989 Specialist Registrar in Oncology, National Cancer Institute, Genova, 1990-1991 Research Fellow and Locum Consultant, National Cancer Institute, 1992 - 2000 Consultant, National Cancer Institute, Genova, Italy (Medical Oncology Department, Cancer Rehabilitation Department)

2000-2002 Consultant, Medical Oncology Department, S.Croce General Hospital, 12/02 - 02/03 Locum Consultant in Medical Oncology, Swansea NHS Trust, Swansea, UK 


\section{Career}

I received primary medical training at the University of Genoa, Italy, obtaining an MD in 1986. I specialized in Oncology and in Internal Medicine at the University of Genoa. I spent 14 years in total at the Genoa National Cancer Institute, first as a Specialist Registrar, then as Research Fellow and Locum Consultant; in 1992 I was appointed fulltime Consultant.

The Genoa National Cancer Institute is one of the country's leading comprehensive cancer centres, offering surgical and non-surgical services at a supra-regional level, with a strong commitment to preclinical and clinical research. Most of my time at the Institute was spent in the Medical Oncology Department, where I gained experience in the management of a wide variety of cancer types, and developed a special interest in breast cancer. The Department has a busy Chemotherapy Day Unit, and in-patient facilities which include beds for high-dose chemotherapy. I also worked for two years in the Cancer Rehabilitation Department of the Institute, where I gained experience in the fields of symptom management and supportive care. In 1997, I spent 3 months at the Breast Unit of the Edinburgh Cancer Centre, supported by a grant from the Italian Association for Cancer Research.

In June 2000 I moved to Cuneo, Italy to cover a post of Consultant Medical Oncologist at the S.Croce General Hospital. This is a 700-bed General Hospital, the main in the southern Piedmont: its Medical Oncology has good in-patient and out-patient facilities, including a Chemotherapy Day Unit which was the first in Italy to obtain ISO 9000 Quality Accreditation. Together with the Radiotherapy and Haematology Departments, it provides non-surgical cancer care to a population of approx. 600.000. Besides my previous interest in breast cancer, during my stay in Cuneo I developed an interest in genito-urinary cancer and pancreatic cancer.

In December 2002 I moved to Swansea, UK to cover a post of Locum Consultant in Medical Oncology. In March 2003 I was appointed full-time Consultant and in July 2003, Honorary Senior Lecturer. The main reason for relocating to Swansea was to join the South West Wales Cancer Institute directed by Prof. Robert Leonard. This offered me the opportunity to work in an exciting environment, where my clinical and academic interests could be developed to their full potentials.

My main clinical responsibilities in Swansea are in the area of breast, genito-urinary (prostate, bladder, testicular) and gynaecological (ovarian) cancer. I have outpatient clinics in Singleton Hospital, Swansea, and in Withybush Hospital, Haverfordwest. I participate to MDT meetings for breast, gynaecological, and genitourinary cancer in Swansea, and through a teleconference link to the breast cancer MDT meeting in Bronglais Hospital, Aberystwyth. 


\section{Academic Activity}

As a Medical Oncologist, I have always been actively involved in clinical research.

During my training at the National Cancer Institute in Genoa, I gained wide experience in the design, conduction, analysis and reporting of clinical trials. In the following years, I have had the opportunity to participate to several national and international phase II-III trials, mainly in the following areas:

- Hormone therapy of breast cancer, including monitoring and treatment of side effects of hormonal drugs

- Chemotherapy and multidisciplinary management of early and advanced breast cancer

- Treatment of other solid tumours (bladder, colorectal, lung, pancreatic and ovarian cancer)

- Supportive care, symptom control and treatment of adverse effects of chemotherapy

My research and academic activity has also included:

- Principal investigator of two multicentre national trials of aromatase inhibitors in breast cancer

- Member of the Steering Committee of the Intergroup Exemestane Study (IES: an international phase III study of adjuvant exemestane vs. tamoxifen in early breast cancer)

- Co-ordinator of a sub-study on endometrial effects in the IES

- Supervision and training of data managers, research nurses, fellows and registrars

My research activity has resulted so far in the authorship or co-authorship of 72 articles in peer-reviewed journals, and of several presentations at conferences.

\section{Professional Societies and Organizations}

Fellow of:

Royal College of Physicians (Edinburgh)

Royal Society of Medicine

Member of:

ASCO (American Society of Clinical Oncology)

ESMO (European Society of Medical Oncology)

SGCTG (Scottish Gynaecological Cancer Trials Group)

ACP (Association of Cancer Physicians)

BMA (British Medical Association)

MPS (Medical Protection Society) 
Publications 


\section{Publications}

- Bertelli G, Paridaens R.

Optimal sequence of hormonotherapy in advanced breast cancer.

Curr Opin Oncol 2006; 18:572-7

- Bertelli G, Garrone O, Merlano M, Occelli M, Bertolotti L, Castiglione F, Pepi F, Fusco O, Del Mastro L, Leonard RC.

Sequential Treatment with Exemestane and Non-Steroidal Aromatase Inhibitors in Advanced Breast Cancer.

Oncology 2005;69:471-477

- Bertelli G, Heouaine A, Arena G, Botto A, Garrone O, Colantonio I, Occelli M, Fea E, Giubergia S, Merlano M.

Weekly docetaxel and zoledronic acid every 4 weeks in hormone-refractory prostate cancer patients.

Cancer Chemother Pharmacol 2006;57:46-51

- Bertelli G.

Sequencing of Aromatase Inhibitors.

Br J Cancer 2005;93 Suppl 1:S6-9

- Bertelli G, Garrone O, Bertolotti L, Occelli M, Conforti S, Marzano N, Febbraro A, Carlini P, Liossi C, Del Mastro L, Leonard RCF.

Maintenance hormone therapy with letrozole after first-line chemotherapy for advanced breast cancer.

Oncology 2005;68:364-370

- Numico G, Colantonio I, Gasco M, Bertelli G, Garrone O, Occelli M, Fea E, Di Costanzo G, Granetto C, Heouaine A, Russi E, Merlano M.

Carboplatin and weekly paclitaxel in non small cell lung cancer patients unfit for or pretreated with chemotherapy.

Anticancer Res 2005;25:2555-9

- Coombes RC, Hall E, Gibson LJ, Paridaens R, Jassem J, Delozier T, Jones SE, Alvarez I, Bertelli G, Ortmann O, Coates AS, Bajetta E, Dodwell D, Coleman RE, Fallowfield LJ, Mickiewicz E, Andersen J, Lonning PE, Cocconi G, Stewart A, Stuart N, Snowdon CF, Carpentieri M, Massimini G, Bliss JM; Intergroup Exemestane Study. A randomized trial of exemestane after two to three years of tamoxifen therapy in postmenopausal women with primary breast cancer.

N Engl J Med. 2004 Mar 11;350(11):108 1-92.

- Menada MV, Papadia A, Lorenzi P, Costantini S, Bertelli GF, Ragni N.

Modification of ultrasonographically measured endometrial thickness after discontinuation of adjuvant therapy with tamoxifen in postmenopausal breast cancer patients.

Eur J Gynaecol Oncol. 2004;25(3):321-3.

- Novarino A, Chiappino I, Bertelli GF, Heouaine A, Ritorto G, Addeo A, Bellone G, Merlano M, Bertetto O.

Phase II study of cisplatin, gemcitabine and 5-fluorouracil in advanced pancreatic cancer.

Ann Oncol 2004 Mar; 15(3):474-7. 
- Catzeddu T, Bertelli G, Del Mastro L, Venturini M.

Sentinel lymph node biopsy in breast cancer patients: The medical oncology perspective.

J Surg Oncol. 2004 Mar;85(3):129-32.

- Bertelli G, Venturini M, Del Mastro L, Bergaglio M, Sismondi P, Biglia N, Venturini S, Porcile G, Pronzato P, Costantini M, Rosso R.

Intramuscular depot medroxyprogesterone versus oral megestrol for the control of postmenopausal hot flashes in breast cancer patients: a randomized study.

Ann Oncol 2002 Jun; 13(6):883-8.

- Valenzano M, Bertelli GF, Costantini S, Corticelli A, Mastro LD, Paoletti R, Garrone $\mathrm{O}$, Rissone R.

Transvaginal ultrasonography and hysterosonography to monitor endometrial effects in tamoxifen-treated patients.

Eur J Gynaecol Oncol 2001;22(6):441-4.

- Bertelli G, Garrone O, Bighin C, Dini D.

Correspondence re: Cicchetti S, Jemec B, Gault DT: two case reports of vinorelbine extravasation: management and review of the literature (Tumori, 86:289-292, 2000).

Tumori 2001 Mar-Apr;87(2):112-3.

- Del Mastro L, Venturini M, Viscoli C, Bergaglio M, Signorini A, Bighin C, Bertelli G, Semino C, Pietra G, Bertoglio S, Sertoli MR, Lambiase A, Rosso R, Melioli G. Intensified chemotherapy supported by DMSO-free peripheral blood progenitor cells in breast cancer patients.

Ann Oncol 2001 Apr;12(4):505-8

- Bertelli G, Queirolo P, Vecchio S, Angiolini C, Bergaglio M, Del Mastro L, Signorini A, Valenzano M, Venturini M.

Toremifene as a substitute for adjuvant tamoxifen in breast cancer patients.

Anticancer Res. 2000 Sep-Oct;20(5C):3659-61.

- Bertelli G, Valenzano M, Costantini S, Rissone R, Angiolini C, Signorini A, Gustavino $\mathrm{C}$.

Limited value of sonohysterography for endometrial screening in asymptomatic, postmenopausal patients treated with tamoxifen.

Gynecol Oncol. 2000 Sep;78(3 Pt 1):275-7.

- Venturini M, Lunardi G, Del Mastro L, Vannozzi MO, Tolino G, Numico G, Viale M, Pastrone I, Angiolini C, Bertelli G, Straneo M, Rosso R, Esposito M.

Sequence effect of epirubicin and paclitaxel treatment on pharmacokinetics and toxicity.

J Clin Oncol. 2000 May;18(10):2116-25.

- Bertelli G, Venturini M, Bergaglio M, Gustavino C, Rosso R, Valenzano M.

Progestins and the endometrium in patients receiving tamoxifen.

Br J Cancer. 1999 Jun;80(7):1114.

- Decensi A, Torrisi R, Gozza A, Severi G, Bertelli G, Fontana V, Pensa F, Carozzo L, Traverso A, Milone S, Dini D, Costa A.

Effect of fenretinide on bone mineral density and metabolism in women with early breast cancer.

Breast Cancer Res Treat. 1999 Jan;53(2):145-51. 
- Dini D, Del Mastro L, Gozza A, Lionetto R, Garrone O, Forno G, Vidili G, Bertelli $G$, Venturini $M$.

The role of pneumatic compression in the treatment of postmastectomy lymphedema. A randomized phase III study.

Ann Oncol. 1998 Feb;9(2):187-90.

- Bertelli G, Venturini M, Del Mastro L, Garrone O, Cosso M, Gustavino C, Cusimano E, Guido T, Nicolo G, Rosso R.

Tamoxifen and the endometrium: findings of pelvic ultrasound examination and endometrial biopsy in asymptomatic breast cancer patients.

Breast Cancer Res Treat. 1998 Jan;47(1):41-6.

- Del Mastro L, Venturini M, Lionetto R, Garrone O, Melioli G, Pasquetti W, Sertoli MR, Bertelli G, Canavese G, Costantini M, Rosso R.

Randomized phase III trial evaluating the role of erythropoietin in the prevention of chemotherapy-induced anemia.

J Clin Oncol. 1997 Jul; 15(7):2715-21.

- Bertelli G, Cafferata MA, Ardizzoni A, Gozza A, Rosso R, Dini D.

Skin ulceration potential of paclitaxel in a mouse skin model in vivo.

Cancer. 1997 Jun 1;79(11):2266-9.

- Pronzato P, Bertelli G, Bruna F, Tani F, Vaira F, Vanoli M, Vigani A.

Intensified M-VEC chemotherapy with G-CSF support as outpatient treatment for advanced bladder cancer.

Anticancer Res. 1997 May-Jun;17(3C):2325-7.

- Del Mastro L, Venturini M, Costantini M, Garrone O, Sertoli MR, Bertelli G, Machi AM, Rosso R.

Paradoxical effect of cytotoxic chemotherapy on anemia in cancer patients with betathalassemia minor.

J Natl Cancer Inst. 1997 Mar 19;89(6):455-6.

- Pronzato P, Bertelli G, Vigani A, Vaira F.

A feasibility study of accelerated polychemotherapy with cisplatin, epidoxorubicin and cyclophosphamide (PEC) in advanced ovarian cancer.

Br J Cancer. 1996 Jun;73(11):1425-7.

- Venturini M, Bruzzi P, Del Mastro L, Garrone O, Bertelli G, Guelfi M, Pastorino S, Rosso R, Sertoli MR.

Effect of adjuvant chemotherapy with or without anthracyclines on the activity and efficacy of first-line cyclophosphamide, epidoxorubicin, and fluorouracil in patients with metastatic breast cancer.

J Clin Oncol. 1996 Mar; 14(3):764-73.

- Pronzato P, Vaira F, Vigani A, Losardo P, Bertelli G.

Biochemical modulation of 5-fluorouracil with methotrexate in advanced colorectal cancer patients pretreated with adjuvant 5-fluorouracil and leucovorin.

Anticancer Res. 1995 Nov-Dec;15(6B):2679-82.

- Bertelli G, Gozza A, Forno GB, Vidili MG, Silvestro S, Venturini M, Del Mastro L, Garrone O, Rosso R, Dini D.

Topical dimethylsulfoxide for the prevention of soft tissue injury after extravasation of vesicant cytotoxic drugs: a prospective clinical study.

J Clin Oncol. 1995 Nov;13(11):2851-5. 
- Sertoli MR, Bruzzi P, Pronzato P, Queirolo P, Amoroso D, Del Mastro L, Venturini $\mathrm{M}$, Vigani A, Bertelli G, Campora E, et al.

Randomized cooperative study of perioperative chemotherapy in breast cancer.

J Clin Oncol. 1995 Nov;13(11):2712-21.

- Pronzato P, Landucci M, Vaira F, Vigani A, Bertelli G.

Carboplatin, methotrexate, and vinblastine in outpatients with advanced transitional cell carcinoma of the bladder.

Am J Clin Oncol. 1995 Jun;18(3):223-5.

- Bertelli G.

Prevention and management of extravasation of cytotoxic drugs.

Drug Saf. 1995 Apr;12(4):245-55

- Dini D, Forno G, Gozza A, Silvestro S, Bertelli G, Toma S, Filippi F, Passarelli B. Combined management in the treatment of epidoxorubicin extravasation. A case report.

Support Care Cancer. 1995 Mar;3(2):150-2.

- Pronzato P, Bruna F, Neri E, Roveri D, Trabucchi A, Vanoli M, Vigani A, Vaira F, Losardo P, Bertelli G.

Radiotherapy plus carboplatin and teniposide in patients with brain metastases from non small cell lung cancer.

Anticancer Res. 1995 Mar-Apr; 15(2):517-9.

- Bertelli G, Dini D, Forno GG, Gozza A.

Preventing cellulitis after axillary lymph node dissection.

Am J Med. 1994 Aug;97(2):202-3.

- Pronzato P, Bertelli G, Losardo P, Landucci M.

What do advanced cancer patients know of their disease? A report from Italy.

Support Care Cancer. 1994 Jul;2(4):242-4.

- Pronzato P, Landucci M, Vaira F, Vigani A, Bertelli G.

Failure of vinorelbine to produce responses in pretreated non-small cell lung cancer patients.

Anticancer Res. 1994 May-Jun;14(3B):1413-5.

- Pronzato P, Landucci M, Vaira F, Vigani A, Bertelli G.

Carboplatin and etoposide as outpatient treatment of advanced non-small-cell lung cancer.

Chemotherapy. 1994 Mar-Apr;40(2):144-8.

- Bertelli G, Dini D, Forno GB, Gozza A, Silvestro S, Venturini M, Rosso R, Pronzato P.

Hyaluronidase as an antidote to extravasation of Vinca alkaloids: clinical results.

J Cancer Res Clin Oncol. 1994;120(8):505-6.

- Pronzato P, Bertelli G, Vaira F, Vigani A, Losardo P.

Mitoxantrone and 5-fluorouracil modulated by the pure (6S) stereoisomer of leucovorin as second-line chemotherapy for advanced breast cancer.

Eur J Cancer. 1994;30A(10):1593-4.

- Pronzato P, Rubagotti A, Amoroso D, Bertelli G, Queirolo P, Sertoli MR, Rosso M, Gallotti P, Monzeglio C, Rosso R.

Second-line hormonotherapy for breast cancer. Uselessness of first-line continuation. Am J Clin Oncol. 1993 Dec;16(6):522-5. 
- Pronzato P, Bertelli G, Rosso M.

A randomized trial on tamoxifen continuation during second-line hormone therapy in breast cancer.

Ann N Y Acad Sci. 1993 Nov 30;698:357-61.

- Pronzato P, Bertelli G, Gardin G, Rubagotti A, Conte PF, Rosso R.

Analysis of time to response to chemotherapy in 316 metastatic breast cancer patients.

Oncology. 1993 Nov-Dec;50(6):460-5.

- Dini D, Macchia R, Gozza A, Bertelli G, Forno GG, Guenzi M, Bacigalupo A, Scolaro T, Vitale V.

Management of acute radiodermatitis. Pharmacological or nonpharmacological remedies?

Cancer Nurs. 1993 Oct;16(5):366-70.

- Dini D, Bertelli G, Gozza A, Forno GG.

Treatment of the post-mastectomy pain syndrome with topical capsaicin.

Pain. 1993 Aug;54(2):223-6.

- Bertelli G, Dini D, Forno G, Gozza A, Venturini M, Ballella G, Rosso R. Dimethylsulphoxide and cooling after extravasation of antitumour agents.

Lancet. 1993 Apr 24;341(8852):1098-9.

- Pronzato P, Miglietta L, Rubagotti A, Bertelli G, Queirolo P, Guenzi M, Sertoli MR, Vitale V, Rosso R.

Impact of irradiation of residual breast on adjuvant chemotherapy dose intensity.

Am J Clin Oncol. 1993 Feb;16(1):58-60.

- Bertelli G, Venturini M, Forno G, Macchiavello F, Dini D.

An analysis of prognostic factors in response to conservative treatment of postmastectomy lymphedema.

Surg Gynecol Obstet. 1992 Nov;175(5):455-60.

- Campora E, Pronzato P, Amoroso D, Bertelli GF, Venturini M, Baldini E, Brunetti I, Sertoli MR, Conte P, Rosso R.

Prognostic factors in node positive primary breast cancer patients treated with adjuvant $\mathrm{CMF}$.

Anticancer Res. 1992 Sep-Oct; 12(5):1555-8.

- Pronzato P, Bertelli G, Mori A, Rosalia Sarcona M, Mortola GP.

Computerized management of therapy protocols in oncology. Evaluation of a decision support for breast carcinoma

Minerva Med. 1991 Nov;82(1 1):733-6.

- Bertelli G, Venturini M, Forno G, Macchiavello F, Dini D.

Conservative treatment of postmastectomy lymphedema: a controlled, randomized trial.

Ann Oncol. 1991 Sep;2(8):575-8.

- Dini D, Venturini M, Forno G, Bertelli G, Grandi G.

Irrigation for colostomized cancer patients: a rational approach.

Int J Colorectal Dis. 1991 Feb;6(1):9-11.

- Pronzato P, Queirolo P, Vidili MG, Amoroso D, Bertelli G, Sertoli MR, Rosso R. Continuous venous infusion of vinblastine in metastatic breast cancer.

Chemotherapy. 1991;37(2):146-9. 
- Pronzato P, Bertelli G, Amoroso D, Pennucci C, Gardin G, Guido T, Rosso P. Oral chemotherapy with idarubicin plus cyclophosphamide in advanced breast cancer.

Chemotherapy. 1991;37(6):449-53.

- Venturini M, Bertelli G, Forno G, Grandi G, Dini D. Colostomy irrigation in the elderly. Effective recovery regardless of age.

Dis Colon Rectum. 1990 Dec;33(12):1031-3.

- Pronzato P, Amoroso D, Bertelli G, Queirolo P, Sertoli MR, Rosso R. Chemotherapy with mitomycin-C, epidoxorubicin and vinblastine in CMF failing breast cancer patients.

Anticancer Res. 1990 Nov-Dec;10(6):1743-5.

- Pronzato P, Brema F, Amoroso D, Bertelli G, Conte PF, Martini MC, Pastorino G, Rosso R.

Megestrol acetate: phase II study of a single daily administration in advanced breast cancer.

Breast Cancer Res Treat. 1990 Nov;17(1):51-4.

- Pronzato P, Bertelli G, Amoroso D, Gozza A, Zaniboni A, Marini G, Rosso R. Cisplatin and recombinant alpha interferon in advanced breast cancer.

Ann Oncol. 1990;1(2):150-1.

- Pronzato P, Campora E, Amoroso D, Bertelli G, Botto F, Conte PF, Sertoli MR, Rosso R.

Impact of administration-related factors on outcome of adjuvant chemotherapy for primary breast cancer.

Am J Clin Oncol. 1989 Dec;12(6):481-5.

- Rosso R, Boccardo F, Brema F, Sertoli MR, Amoroso D, Bertelli G, Pronzato P. Adjuvant systemic treatment of resectable breast cancer: eleven years results of a monoinstitutional chemo-hormone-immunotherapy trial.

Anticancer Res. 1989 Jul-Aug;9(4):1153-6.

- Pronzato P, Amoroso D, Bertelli G, Conte PF, Cusimano MP, Ciottoli GB, Gulisano $\mathrm{M}$, Lionetto R, Rosso R.

Phase II study of lonidamine in metastatic breast cancer.

Br J Cancer. 1989 Feb;59(2):251-3.

- Amoroso D, Bertelli G, Pronzato P, Vidili MG, Gulisano M, Lionetto R, Rosso R. Continuous venous infusion of vindesine in metastatic breast cancer: experience with a subcutaneously implanted system and portable pump.

Anticancer Res. 1989 Jan-Feb;9(1):141-3.

- Canavese G, Catturich A, Battistini G, Caroti C, Pronzato P, Gardin G, Amoroso D, Bertelli G, Conte PF, Tomao S, et al.

The role of surgery in the combined treatment of locally advanced breast cancer. Oncology. 1989;46(6):353-6.

- Bertelli G, Pronzato P, Amoroso D, Cusimano MP, Conte PF, Montagna G, Bertolini

S, Rosso R.
Adjuvant tamoxifen in primary breast cancer: influence on plasma lipids and antithrombin III levels.

Breast Cancer Res Treat. 1988 Dec;12(3):307-10. 
- Bertelli G, Amoroso D, Pronzato P, Rosso R.

Idarubicin: an evaluation of cardiac toxicity in 77 patients with solid tumors.

Anticancer Res. 1988 Jul-Aug:8(4):645-6.

- Amoroso D. Pronzato P, Bertelli G, Gallotti P. Pastorino G, Cusimano MP, Merlano M. Conte PF, Rosso R.

Cisplatin and 5-fluorouracil in refractory breast cancer patients: a phase II study.

Breast Cancer Res Treat. $1988 \mathrm{Jul} ; 1$ 1(3):269-71.

- Campora E, Repetto L, Giuntini P, Bertelli G, Amoroso D, Sertoli MR, Rosso R. $\mathrm{LDH}$ in the follow-up of stage I malignant melanoma.

Eur J Cancer Clin Oncol. 1988 Feb:24(2):277-8.

- Conte PF, Alama A, Bertelli G, Canavese G, Carnino F, Catturich A, Di Marco E, Gardin G, Jacomuzzi A, Monzeglio C, et al.

Chemotherapy with estrogenic recruitment and surgery in locally advanced breast cancer: clinical and cytokinetic results.

Int J Cancer. 1987 Oct 15:40(4):490-4.

- Pronzato P. Amoroso D, Ardizzoni A. Bertelli G, Conte PF, Michelotti A, Rosso R. A phase II study with danazol in metastatic breast cancer.

Am J Clin Oncol. 1987 Oct:10(5):407-9.

- Pronzato P, Amoroso D, Ardizzoni A, Bertelli G, Canobbio L, Conte PF, Cusimano MP, Fusco V, Gulisano M, Lionetto R, et al.

Sequential administration of cyclophosphamide, methotrexate, 5-fluorouracil, and folinic acid as salvage treatment in metastatic breast cancer.

Am J Clin Oncol. 1987 Oct;10(5):404-6.

- Repetto L, Pronzato P, Ardizzoni A, Bertelli G, Gulisano M, Amoroso D, Gardin G, Fusco V, Falcone A, Conte PF, et al.

Metastatic breast cancer: an analysis of prognostic factors in patients treated with aminoglutethimide.

Chemioterapia. 1987 Feb;6(1):66-72.

- Lionetto R, Pronzato P, Conte PF, Amoroso D, Badellino F, Bertelli G, Canavese G, Gardin G, Rosso R.

Multimodality treatment of locally advanced breast cancer.

Oncology. 1987:44(3):137-41.

- Lionetto R, Pronzato P, Bertelli GF, Ardizzoni A, Conte PF, Rosso R.

Survival of patients with relapsing breast cancer: analysis of 302 patients.

Oncology. 1986:43(5):278-82. 